arXiv: 0810.5195

UPR 1201-T

\title{
Abelian Fibrations, String Junctions, and Flux/Geometry Duality
}

\author{
Ron Donagi, ${ }^{a *}$ Peng Gao ${ }^{b \dagger}$ and Michael B. Schulz ${ }^{c \ddagger}$ \\ ${ }^{a}$ Department of Mathematics, University of Pennsylvania \\ Philadelphia, PA 19104, USA \\ ${ }^{b}$ Departments of Physics, University of Toronto \\ Toronto, Ontario, Canada MSS 1A7 \\ ${ }^{c}$ Department of Physics, Bryn Mawr College \\ Bryn Mawr, PA 19010, USA
}

\begin{abstract}
In previous work, it was argued that the type IIB $T^{6} / \mathbb{Z}_{2}$ orientifold with a choice of flux preserving $\mathcal{N}=2$ supersymmetry is dual to a class of purely geometric type IIA compactifications on abelian surface $\left(T^{4}\right)$ fibered Calabi-Yau threefolds. We provide two explicit constructions of the resulting Calabi-Yau duals. The first is a monodromy based description, analogous to F-theory encoding of Calabi-Yau geometry via 7-branes and string junctions, except for $T^{4}$ rather than $T^{2}$ fibers. The second is an explicit algebro-geometric construction in which the $T^{4}$ fibers arise as the Jacobian tori of a family of genus- 2 curves. This improved description of the duality map will be a useful tool to extend our understanding of warped compactifications. We sketch applications to related work to define warped Kaluza-Klein reduction in toroidal orientifolds, and to check the modified rules for D-brane instanton zero mode counting due to the presence of flux and other D-branes. The nontrivial fundamental groups of the Calabi-Yau manifolds constructed also have potential applications to heterotic model building.
\end{abstract}

29 October 2008

\footnotetext{
*donagi at math.upenn.edu

${ }^{\dagger}$ gaopeng at physics.utoronto.ca

${ }^{\ddagger}$ mbschulz at brynmawr.edu
} 


\section{Contents}

1 Introduction $\quad 3$

2 Monodromy and junction description of elliptic fibrations 6

2.1 F-theory . . . . . . . . . . . . . . . . . . . 7

2.1.1 The F-theory limit . . . . . . . . . . . . . . . . . 7

2.1.2 Singular fibers, $(p, q)$ 7-branes, and $(p, q)$ strings . . . . . . . . 7

$2.2 \quad$ F-theory on $\mathrm{K} 3 \ldots \ldots \ldots \ldots$

2.3 Monodromies and braiding . . . . . . . . . . . . . . . . . 9

2.4 String junctions and gauge symmetry . . . . . . . . . . . . . . . . . 11

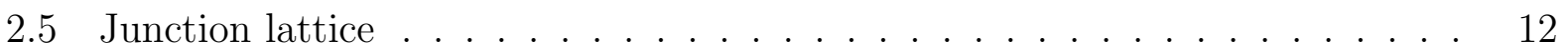

2.5.1 Junction lattice of $\frac{1}{2} \mathrm{~K} 3=\mathrm{dP}_{9} \ldots \ldots \ldots$. . . . . . . . . . 15

2.5.2 Mathematical interpretation of the junction lattice . . . . . . . . 17

2.5.3 An example with coalesced 7-branes . . . . . . . . . . . . . . 19

2.6 Weakly integral junction lattice and torsion sections . . . . . . . . . 20

3 Monodromy and junction description of CY duals of $T^{6} / \mathbb{Z}_{2} \quad 23$

3.1 The duality map . . . . . . . . . . . . . . . . . . . . . . 23

3.2 Known properties of type IIA Calabi-Yau duals $\mathcal{X}_{m, n} \ldots \ldots$. . . . . . . . . 24

3.3 Monodromy matrices for the abelian surface fibrations . . . . . . . . . . 26

3.3.1 Fundamental group . . . . . . . . . . . . . . . 27

3.3.2 Calabi-Yau dual interpretation of $T^{6} / \mathbb{Z}_{2}$ RR tadpole . . . . . . . . 28

3.4 Mordell-Weil lattice from junction lattice . . . . . . . . . . . . . . . . . . 28

3.5 Examples with coalesced fibers . . . . . . . . . . . . . . . . . 31

3.5.1 $\mathbb{Z}_{2 m} \oplus \mathbb{Z}_{m}$ torsion, $m=1,2,3 \ldots \ldots \ldots \ldots \ldots$

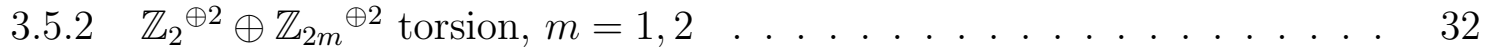

3.5.3 $\mathbb{Z}_{4} \oplus \mathbb{Z}_{2}{ }^{\oplus 2}$ torsion and $\mathbb{Z}_{2}^{\oplus 3}$ torsion . . . . . . . . . . . . . 33

3.6 Connections to other Calabi-Yau manifolds with nontrivial $\pi_{1}$. . . . . . . . 34

4 Algebraic construction in the principally polarized case 36

4.1 The surface $S \ldots \ldots \ldots \ldots$. . . . . . . . . . . . . . . . . . . . . . . .

4.2 The 3 -fold $\mathcal{X} \ldots \ldots \ldots \ldots$. . . . . . . . . . . . . . . . . . . . . . 39

4.3 Checks . . . . . . . . . . . . . . . . . . . . . . 41

4.3.1 Intersection numbers . . . . . . . . . . . . . . . . . . 41

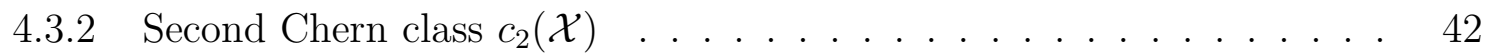

4.3 .3 Mordell-Weil lattice: $D_{12}^{-} \ldots \ldots \ldots$. . . . . . . . . . 43

4.4 Mordell-Weil torsion and connection to other CY manifolds . . . . . . . . . . 44

$\begin{array}{lll}5 & \text { Conclusions and future directions } & 47\end{array}$ 
$\begin{array}{ll}\text { A Braiding operations and monodromy matrices } & \mathbf{5 0}\end{array}$

A.1 Elliptic fiber . . . . . . . . . . . . . . . . . . . . 50

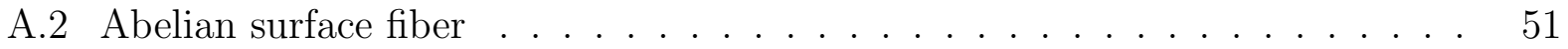

B Complex tori, abelian varieties, and the Mordell-Weil lattice 51

$\begin{array}{ll}\text { C Mordell-Weil height pairing from intersections } & 54\end{array}$

C.1 Elliptic fibration over a curve . . . . . . . . . . . . . . . . 54

C.2 Abelian surface fibration over a curve . . . . . . . . . . . . . 56

$\begin{array}{ll}\text { D Monodromy matrices for the abelian fibration } \mathcal{X}_{m, n} & 56\end{array}$

$\begin{array}{lll}\text { E Null loop junctions of } \mathcal{X}_{m, n} & 60\end{array}$

$\begin{array}{lll}\text { F Complex curves and their Jacobians } & 62\end{array}$

G Genera of curves in $\mathbb{P}^{1} \times \mathbb{P}^{1} \quad 64$

$\begin{array}{ll}\text { H Direct image functor } & 65\end{array}$

$\begin{array}{lll}\text { I Proof of the Calabi-Yau condition } & 67\end{array}$

$\begin{array}{lll}\text { J Intersections of theta surfaces of } \mathcal{X} & 68\end{array}$ 


\section{Introduction}

An enduring theme in string theory has been duality - the existence of dissimilar, but nonetheless equivalent discriptions of the same physical string theory vacuum. One particularly fruitful avenue has been open/closed string duality, which relates the physics of D-branes and open strings to purely geometrical closed string backgrounds. In this way, we have gained insight into strongly coupled gauge theories from geometry, and into the geometry of special holonomy manifolds from consistent D-brane constructions.

Given the prominence of type IIB flux compactifications in string theory model building, it seems useful to ask whether duality might again be put to productive use to address shortcomings in our understanding of this class of vacua. For example: Flux compactifications are warped compactifications with D-branes and internal magnetic flux. The word "warped" means that a scale factor governing the overall size of $4 \mathrm{D}$ spacetime varies from point to point in the $6 \mathrm{D}$ compact extra dimensions. In contrast to standard compactifications, where there is a well defined Kaluza-Klein (KK) procedure for extracting the 4D effective field theory from 10D, there is at present only a partial understanding of the analogous procedure for warped compactifications $[19,26,22,10,53]$. Duality is one route through which a definition can be sought.

In the context of $\mathcal{N}=2$ supersymmetry, it was shown in Ref. [46] that the simplest class of IIB flux compactifications, the type $T^{6} / \mathbb{Z}_{2}$ orientifold with D3 branes and $\mathcal{N}=2$ flux, is dual to a class of purely geometric type IIA Calabi-Yau compactifications with no warping, no D-branes and no flux. ${ }^{1} T^{6} / \mathbb{Z}_{2}$, while too simplistic to itself form the basis for a realistic model, has a good track record as a source of insight into flux compactifications. It was the setting for the first global analysis of moduli stabilization from flux [37, 21], and a point of departure for the study of de Sitter vacua [44] and other forms of NS sector flux [38, 45, 49, 39], including twists of topology and nongeometric flux [33]. A deeper understanding of the duality map between $T^{6} / \mathbb{Z}_{2}$ and conventional type IIA Calabi-Yau compactifications stands to shed insight into warped compactifications in general. However, a prerequisite is a precise understanding of both sides of the duality map.

The purpose of the work reported here is to explicitly construct the type IIA Calabi-Yau compactifications dual to $T^{6} / \mathbb{Z}_{2}$, and to make more precise the encoding of their topology and geometry by the dual configuration of D-branes and flux. The Calabi-Yau manifolds $\mathcal{X}_{m, n}$ that arise are abelian surface $\left(T^{4}\right)$ fibrations over $\mathbb{P}^{1}$. Many of their topological properties were deduced in Ref. [46], however an explicit construction was left for future work. We provide two such constructions here.

The first construction is in terms of monodromy matrices of the abelian fibrations $\mathcal{X}_{m, n}$.

\footnotetext{
${ }^{1} \mathrm{~A}$ similar duality was explored in Ref. [2], where it was shown that the class of F-theory compactifications on $\mathrm{K} 3 \times \mathrm{K} 3$ with $\mathcal{N}=2$ flux is again dual to purely geometric Calabi-Yau compactifications of type IIA string theory. In this case, the resulting manifolds are K3 fibered.
} 
This construction is largely inspired by the work [15, 16, 17, 18, 23] on string junctions in F-theory and its relation to the geometry of elliptic surfaces. Our work generalizes this formalism to the case of $T^{4}$ rather than $T^{2}$ fiber. We show that the D3 tadpole condition in $T^{6} / \mathbb{Z}_{2}$ maps to the condition that the total monodromy about all singular fibers of $\mathcal{X}_{m, n}$ is unity. Building on Ref. [23], we show that the junction formalism is again an efficient means to compute Mordell-Weil lattice of sections of the abelian fibration in the $T^{4}$ fibered case. For the Calabi-Yau manifold $\mathcal{X}_{m, n}$, we show that the free component of the Mordell-Weil lattice is the $D_{M}$ root lattice, where $M=16-4 m n$ is the number of D3-branes in $T^{6} / \mathbb{Z}_{2}$. The generic torsion component of the Mordell-Weil group is $\mathbb{Z}_{m} \times \mathbb{Z}_{m}$, in agreement with the isometry group inferred in Ref. [46]; at special points in moduli space it is enhanced to a larger discrete group, characterized by the lattice of weakly integral null junctions. We are also able to use the monodromy description to verify the $\mathbb{Z}_{n} \times \mathbb{Z}_{n}$ fundamental group deduced by duality in Ref. [46]. Finally, quotienting by isometries gives a general way to construct new Calabi-Yau manifolds with nontrivial fundamental group.

The second construction takes an explicit algebraic geometry approach. In the case, we begin with an auxilliary surface $S$ that is fibered by genus- 2 curves over $\mathbb{P}^{1}$. By replacing each genus-2 curve with its Jacobian torus, we obtain a 3-fold that we show is CalabiYau. Its homology, interection numbers, Mordell-Weil lattice and second Chern class all agree with those of $\mathcal{X}_{1,1}$. Therefore, the two are equivalent up to homotopy type by Wall's theorem [35, 54]. Finally, we again explore the enhancement of Mordell-Weil torsion (i.e., the isometry group) at special loci in moduli space in this framework, and connect to a subset of the results obtained from the junction description.

This work, together with Ref. [46], lays the groundwork for the following applications, to be reported in separate articles, as sketched in Sec. 5:

i. to define warped KK reduction for simple warped compactifications by duality to conventional Calabi-Yau compactifications [7].

ii. in the context of D3-brane instantons, to check the modified rules for instanton zero mode counting due to flux or intersections with other localized objects, by duality to worldsheet instantons in type IIA [25].

As a byproduct, the following application arises, as explained in Sec. 3.6:

iii. to construct examples of new Calabi-Yau manifolds with nontrivial fundamental group of interest for heterotic model building [20, 43]. As highlighted in Refs. [31, 29, 30, 12, $8,9]$, very few Calabi-Yau manifolds with nontrivial fundamental group are explicitly known.

An outline of the paper is as follows: 
In Sec. 2, we review the monodromy and junction description of elliptic fibrations. This establishes the background and point of view in preparation for an analogous description of abelian surface fibered Calabi-Yau manifolds in Sec. 3. For simplicity, we focus on the case of $\mathbb{P}^{1}$ base. The topology of a generic elliptic fibration over $\mathbb{P}^{1}$ is defined by a collection of points on the $\mathbb{P}^{1}$ and the monodromies about the $\mathrm{I}_{1}$ singular fibers at these points. The collection of monodromies is unique up to braiding operations and overall $S L(2, \mathbb{Z})$ conjugation. Via the F-theory, this is the same data that determines a collection of 7-branes in a type IIB compactification on the base of the elliptic fibration, and their $(p, q)$ types. The $W$-bosons of the spontaneously broken gauge theory on the 7-branes are string junctions terminating on 7-branes. Each can be represented by a tree graph on the base, and encodes a curve in the elliptic fibration with zero intersection with the fiber and base. Following Refs. [15, $16,17,18]$, we explain how various coalescing collections of 7-branes realize unbroken ADE type gauge symmetries. Finally, following Ref. [23], we describe how the junction lattice determines the Mordell-Weil group of rational sections of the elliptic fibration. ${ }^{2}$

In Sec. 3, we review the duality map between the type IIB $T^{6} / \mathbb{Z}_{2}$ orientifold with $\mathcal{N}=2$ flux and type IIA compactified on the Calabi-Yau manifolds $\mathcal{X}_{m, n}$. Then, we generalize the mondoromy and junction description of the previous sections to be applicable to abelian surface fibrations, focusing on the $\mathcal{X}_{m, n}$. In particular, we determine collections of $S L(4, \mathbb{Z})$ monodromy matrices that define the topology of the $\mathcal{X}_{m, n}$. The condition that the total monodromy be unity reproduces the D3 charge cancellation condition of $T^{6} / \mathbb{Z}_{2}$. The $\mathcal{X}_{m, n}$ are abelian surface fibrations over $\mathbb{P}^{1}$, where an abelian surface is a $T^{4}$ that admits an embedding in complex projective space. The latter endows the $T^{4}$ with a Hodge form (or equivalently, a theta divisor), which is precisely the additional ingredient necessary to define an inner product and give the space of junctions the structure of a lattice. This lattice again determines the Mordell-Weil group of sections, including torsion, and the torsion subgroup is an isometry group of the Calabi-Yau manifold. We describe its enhancement at singular loci in moduli space in terms of weakly integral null junctions that become relevant when $\mathrm{I}_{1}$ fibers coalesce. Quotienting by these isometries gives new Calabi-Yau manifolds with nontrivial fundamental group.

In Sec. 4 , we provide an algebro-geometric construction of $\mathcal{X}_{1,1}$ as the relative Jacobian of a genus- 2 fibration over $\mathbb{P}^{1}$. Every smooth principally polarized abelian surface is the Jacobian of some genus- 2 curve. Therefore, in the principally polarized cases $m=n$, we might expect to realize the Calabi-Yau threefold $\mathcal{X}_{m, n}$ as the relative (i.e., fiberwise) Jacobian of an auxilliary surface fibered by genus- 2 curves. We show that this is indeed the case. Since a genus- 2 curve is the double cover of $\mathbb{P}^{1}$ with 6 branch points, we consider a surface $S$ that is the double cover of $\mathbb{P}^{1} \times \mathbb{P}^{1}$ branched over a $(6,2)$ curve $B$. The relative Jacobian $\mathcal{J}_{S / \mathbb{P}^{1}}$

\footnotetext{
${ }^{2}$ To be precise, we determine both the Mordell-Weil group and the Mordell-Weil lattice. The Mordell-Weil group includes the torsion sections, but not the lattice inner product. The Mordell-Weil lattice includes the lattice inner product, but not the torsion sections.
} 
indeed reproduces the Calabi-Yau manifold $\mathcal{X}_{1,1}$. After verifying the Calabi-Yau condition, we show that the Hodge numbers, intersection numbers, and second Chern class of $\mathcal{J}_{S / \mathbb{P}^{1}}$ match those of $\mathcal{X}_{1,1}$. These are the classifying data of a Calabi-Yau threefold up to homotopy type, by Wall's theorem and its extensions. Finally, we compute the Mordell-Weil lattice and show that it matches as well. At special loci in moduli space, where the branch curve $B$ factorizes, we compute the Mordell-Weil torsion, and reproduce some of the results of Sec. 3.

We conclude with summary of results and a discussion of connections to related and ongoing work. Ongoing work by the authors include applications to warped KK reduction, D-brane instanton corrections, heterotic model building, and $S U(2)$ stucture Calabi-Yau compactifications, where the topology of an abelian surface fibration spontaneously breaks extended supersymmetry. We also outline connections to recent work on D(imensional) duality [27] and semi-flat T-fold compactifications [36, 56].

Derivation of key results and relevant mathematical and physical background can be found in the appendices. App. A describes how the monodromies of branes or singular fibers are transformed under braiding motions of the locations of these objects. The duality derivation of the monodromy matrices of $\mathcal{X}_{m, n}$ is given in App. D, and the lattice vectors of null loop junctions are computed in App. E. Apps. B and C contain background on abelian varieties and the Mordell-Weil lattice. App. F is an introduction to complex curves, their Jacobians, and line bundles. App. $\mathrm{H}$ gives background on direct images. Finally, Apps. G, $\mathrm{I}$ and $\mathrm{J}$ contain the derivations of mathematical results used in Sec. 4.

\section{Monodromy and junction description of elliptic fibrations}

Given an elliptically fibered ${ }^{3}$ Calabi-Yau manifold $\mathcal{X}$ with base $\mathcal{B}$, F-theory provides a nonperturbative definition of a family of type IIB string theory vacua with spatially varying dilaton-axion $\tau$ [55]. The type IIB vacua are defined by "Newton's Law," $F=\left.M\right|_{A_{T^{2}} \rightarrow 0}$ $[55,3]$. That is, we consider M-theory on $\mathcal{X}$ in the limit that the area of the elliptic fiber goes to zero, while holding the complex structure fixed. The result is type IIB compactified on $\mathcal{B}$, with $\tau$ identified with the complex structure modulus of the elliptic fiber at each point on $\mathcal{B}$. The codimension 1 singular locus of the elliptic fibration on $\mathcal{B}$ is wrapped by 7 -branes in the IIB description, where the type of each 7-brane is determined by the monodromy of $\tau$ about the 7-brane worldvolume.

Conversely, the nonperturbative type IIB description provides a useful encoding of the geometry of $\mathcal{X}$. The collection of $(p, q)$ 7-branes determines the degenerations of the elliptic fibration and the corresponding monodromy matrices about singular fibers. These data determine the topology of $\mathcal{X}$. Additional geometric information is efficiently encoded by string junctions terminating on the 7-branes. These string junctions are the $W$-bosons of

\footnotetext{
${ }^{3}$ Elliptic and abelian surface fibrations always refer to fibrations with section in this paper.
} 
the 7-brane gauge theory. Their equivalence classes form a charge lattice known as the junction lattice. The string junctions lift to M2-branes wrapped on 2-cycles of $\mathcal{X}$, so they encode information about the geometry of 2-cycles. For example, enhanced gauge symmetry corresponds to coalescing groups of 7 -branes in IIB. In this case, the massless $W$-bosons are string junctions contractible to zero length, which lift to 2-cycles contractible to zero volume. Roughly speaking, in the case of $\mathbb{P}^{1}$ base, $H_{2}(\mathcal{X})$ comes from the generic fiber, extra components of singular fibers, and sections of the elliptic fibration $\pi: \mathcal{X} \rightarrow \mathbb{P}^{1}$. The string junctions are related to the latter. As we will see in Sec. 2.5.2, the junction lattice determines the Mordell-Weil lattice of rational sections of the elliptic fibration [23].

To describe the geometry of the Calabi-Yau duals of $T^{6} / \mathbb{Z}_{2}$ in Sec. 3, we apply a similar monodromy and junction based description to the case of $T^{4}$ rather than $T^{2}$ fiber. With this goal in mind, the remainder of this section is devoted to laying the groundwork for the generalization by analyzing the simpler elliptically fibered case in more detail.

\section{$2.1 \quad$ F-theory}

\subsubsection{The F-theory limit}

Let us briefly review the duality chain and limit that relates the initial M-theory background to the final type IIB background. We begin with M-theory compactified on an elliptic fibration $\mathcal{X}$ over base $\mathcal{B}$. The generic fiber has two nontrivial 1 -cycles $\alpha$ and $\beta$. In the limit of small $R_{\alpha}$, the background is described by perturbative type IIA string theory with ${ }^{4}$ $g_{s}^{\mathrm{IIA}}=R_{\alpha} \ll 1$. The IIA compactification manifold is the base of the $S_{\alpha}^{1}$ fibration. If $R_{\beta}$ is also small, then it is appropriate to T-dualize to type IIB. In the $R_{\beta} \rightarrow 0$ limit, the type IIB $\beta$ cycle decompactifies, leaving type IIB compactified on the base $\mathcal{B}$.

For the special case of a rectangular torus, the type IIB dilaton-axion is purely imaginary and can be identified with the complex structure modulus of the elliptic fiber: $i / g_{s}^{\mathrm{IIB}}=$ $i R_{\beta}^{\mathrm{IIA}} / g_{s}^{\mathrm{IIA}}=i R_{\beta} / R_{\alpha}=\tau$. For a nonrectangular torus, the identification remains valid and the real part of the complex structure modulus gives nonzero type IIB axion $C_{(0)}$.

\subsubsection{Singular fibers, $(p, q)$ 7-branes, and $(p, q)$ strings}

For now we assume that degenerations of the elliptic fibration $\mathcal{X}$ are of Kodaira type $\mathrm{I}_{1}$ : a $(p, q)$ 1-cycle $p \alpha+q \beta \subset T^{2}$ vanishes over a codimension 1 locus $D$ in the base. In the corresponding type IIB interpretation, a $(p, q)$ 7-brane wraps the divisor $D \subset \mathcal{B}$ and spans the noncompact dimensions of spacetime (see Fig. 1). Here, a $(p, q) 7$-brane is an object on which a $(p, q)$ string can end. ${ }^{5}$ Thus, a $(1,0) 7$-brane is a D7-brane.

\footnotetext{
${ }^{4}$ Here, we assume unit periodicity $x \cong x+1$ for toroidal coordinates and set $2 \pi \sqrt{\alpha^{\prime}}=1$ for simplicity.

${ }^{5} \mathrm{~A}(p, q)$ string is the bound state of $p$ fundamental and $q$ D-strings. The space of all $(p, q)$ strings, with $p$ and $q$ relatively prime, is the $S L(2, \mathbb{Z})$ S-duality orbit of a fundamental string.
} 
The F-theory limit relates the dual interpretations of the integers $(p, q)$ as string charge and homology vector. A $(p, q)$ string in type IIB lifts to an M-theory membrane wrapped on a $p \alpha+q \beta$ cycle in the fiber of $\mathcal{X}$.

Type IIB:

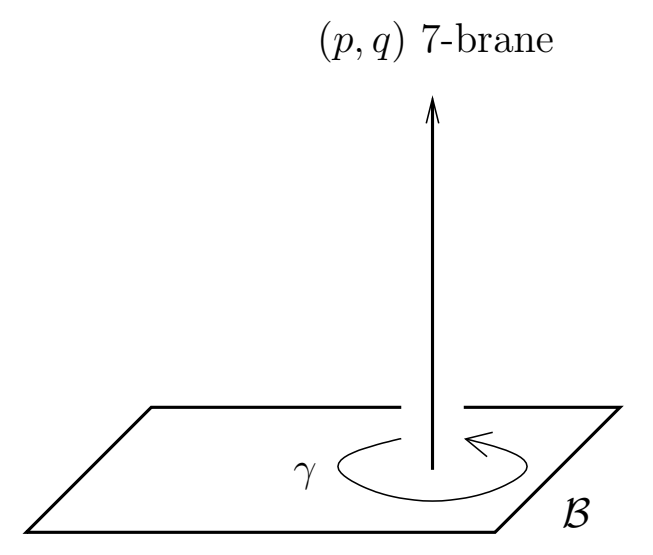

$\mathcal{X}$ : generic $T^{2}$ fiber

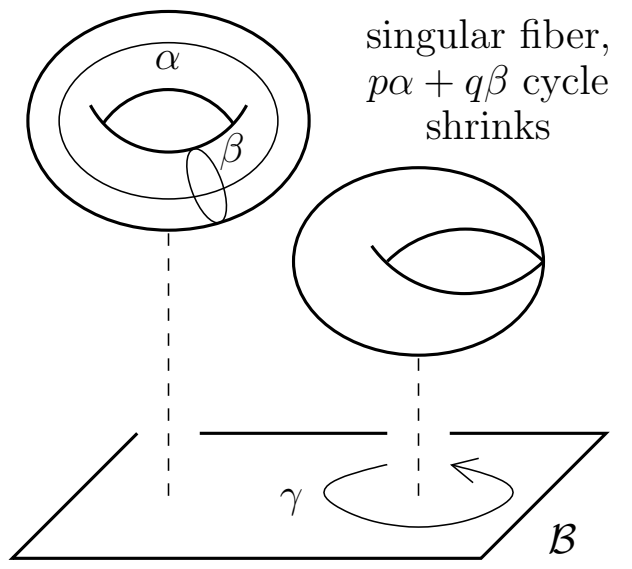

Figure 1: F-theory relates a $(p, q) 7$-brane in type IIB to an elliptic fiber with vanishing $(p, q)$-cycle in $\mathcal{X}$.

\section{$2.2 \quad$ F-theory on $\mathrm{K} 3$}

The simplest F-theory compactification manifold with nontrivial base is a K3 surface. A generic elliptic K3 surface is an elliptic fibration over a $\mathbb{P}^{1}$ base, with $24 \mathrm{I}_{1}$ singular fibers over points on $\mathbb{P}^{1}$. The corresponding IIB background is a compactification of type IIB string theory on $\mathbb{P}^{1}$ with $24(p, q)$ 7-branes, each located at a point on $\mathbb{P}^{1}$ and filling the $7+1$ noncompact dimensions of spacetime.

The weak coupling perturbative interpretation of this background was well known long before the formulation of F-theory. It is the $T^{2} / \mathbb{Z}_{2}$ orientifold, dual to type I string theory via T-duality in the two $T^{2}$ directions. There are 16 D7-branes at arbitrary points on $T^{2} / \mathbb{Z}_{2} \cong \mathbb{P}^{1}$ and 4 O7-planes located at the fixed points of the $\mathbb{Z}_{2}$ involution. A total of $16+4=20$ objects is too few to correspond to the $24(p, q) 7$-branes required by F-theory. However, it is already clear from the perturbative description that the O7-planes are poorly described by leading order supergravity and thus need not represent fundamental objects $[48,45,46]$. At finite distance from the $\mathrm{O} 7$ planes, the string coupling diverges. The geometry is a warped product $\mathbb{R}^{7,1} \rtimes \mathbb{P}^{1}$ in which a $\mathbb{P}^{1}$ dependent scale factor multiplies the flat metric on $\mathbb{R}^{7,1}$, and this scale factor diverges at finite distance from the O7-planes. 
F-theory provides an elegant nonperturbative resolution of this pathology. Each O7-plane resolves to a pair of $(p, q)$ 7-branes. Up to braiding operations on the 7-branes described below, the pair is $(p, q)=(1,-1)$ and $(1,1)$. The separation between the two 7-branes depends on the string coupling as $\exp \left(-1 / g_{s}\right)$, so it is invisible in perturbation theory. ${ }^{6}$ This phenomenon can also be described by $S U(2)$ Seiberg-Witten theory: $\mathcal{N}=2, S U(2)$ superYang-Mills theory is the theory on D3-brane probe in the background of an O7-plane [4]. In the classical gauge theory moduli space, there is an enhanced $S U(2)$ symmetry point where the D3-brane coincides with the O7-plane. Quantum mechanically (due to instanton corrections in the gauge theory), the $S U(2)$ point is lifted and replaced by two massless dyon points separated by a distance of order $\exp \left(-4 \pi / g_{\mathrm{YM}}^{2}\right)[47]$. The two dyon hypermultiplets are $(p, q)$ strings stretched from the D3 probe to the $(p, q)=(1, \pm 1) 7$-branes. A dyon becomes massless when the D3-brane is coincident with the corresponding 7-brane.

\subsection{Monodromies and braiding}

Three types of $(p, q)$ 7-branes appeared in the type IIB description of F-theory on K3 given in the previous section. Their $(p, q)$ charges are $\mathbf{A}=(1,0), \mathbf{B}=(1,-1)$ and $\mathbf{C}=(1,1)$. In this notation, a D7-brane is an $\mathbf{A}$ brane located at a point on $\mathbb{P}^{1}$ and an O7-plane resolves to a $\mathbf{B}, \mathbf{C}$ pair.

\begin{tabular}{|c|c|c|c|c|c|c|c|c|c|}
\hline$A$ & A & B & $\mathrm{C}$ & B & $\mathrm{C}$ & B & $\mathrm{C}$ & B & $\mathrm{C}$ \\
\hline 1 & 1 & 1 & 1 & 1 & 1 & 1 & 1 & 1 & 1 \\
\hline 1 & 1 & 1 & 1 & 1 & 1 & 1 & 1 & 1 & 1 \\
\hline I & I & I & 1 & 1 & 1 & 1 & I & 1 & 1 \\
\hline 1 & 1 & 1 & 1 & 1 & 1 & 1 & 1 & 1 & 1 \\
\hline 1 & 1 & 1 & 1 & 1 & 1 & 1 & 1 & 1 & 1 \\
\hline 1 & 1 & 1 & 1 & 1 & 1 & 1 & 1 & 1 & 1 \\
\hline | & 1 & 1 & 1 & 1 & 1 & 1 & 1 & 1 & I \\
\hline
\end{tabular}

Figure 2: A collection $\mathbf{A}, \mathbf{B}, \mathbf{C}$ points in $\mathbb{P}^{1}$, and their branch cuts. The collection consists of $16 \mathbf{A}$ points and $4 \mathbf{B}, \mathbf{C}$ pairs.

We can represent the IIB background, or equivalently the K3 surface, by arranging the $\mathbf{A}, \mathbf{B}$ and $\mathbf{C}$ points on the projective plane $\mathbb{P}^{1}$, keeping track of the locations of branch cuts (see Fig. 2). Here, the branch cuts denote discontinuities in $\tau$. If we do not introduce branch cuts, then $\tau$ is multiple-valued. For example, $\tau \rightarrow \tau+1$ after circuiting a D7-brane counterclockwise, since $\operatorname{Re} \tau=C_{(0)}$ and since a D7-brane is a source of Ramond-Ramond (RR) flux $F_{(1)}=d\left(C_{(0)}\right)$. Alternatively, we can opt for a single valued RR potential $C_{(0)}$ plus a Dirac string, that is, a branch cut. Then, the discontinuity in crossing the branch cut of

\footnotetext{
${ }^{6}$ From the M-theory perspective, the corrections come from Kaluza-Klein modes along the circles in the elliptic fiber. From the IIB perspective, the corrections come from $(p, q)$-strings, whereas the leading supergravity description only contains the effective field theory of a fundamental string.
} 
a D7-brane counterclockwise is $\tau \rightarrow \tau-1$. There are similar, $S L(2, \mathbb{Z})$ dual, discontinuities along the branch cuts of other $(p, q)$ 7-branes. Let us agree to draw all branch cuts as vertical lines intersecting at the point at infinity on $\mathbb{P}^{1}$. This determines an ordering (left to right in Figs. 2 and 3) of the 7-branes, which we summarize in the diagram of Fig. 2, or more compactly as

\section{$\mathrm{A}^{16} \mathrm{BC}$ BC BC BC.}

We denote an arbitrary $(p, q)$ 7-brane (or singular fiber) by $\mathbf{X}_{[p, q]}$, so for a more general collection of $(p, q)$ 7-branes,

$$
\mathbf{X}_{\left[p_{1}, q_{1}\right]} \mathbf{X}_{\left[p_{2}, q_{2}\right]} \ldots \mathbf{X}_{\left[p_{n}, q_{n}\right]}
$$

we have a diagram of the form shown in Fig. 3.

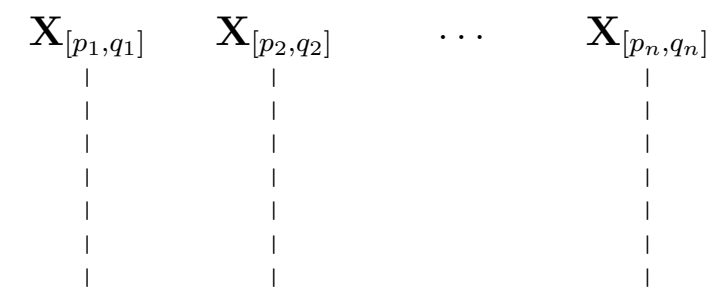

Figure 3: A collection of $\mathbf{X}_{[p, q]}$ points in $\mathbb{P}^{1}$, and their branch cuts.

We have already observed that a $(p, q)$ string can end at $(p, q) 7$-brane. When a $\left(p^{\prime}, q^{\prime}\right)$ string crosses a branch cut of a $(p, q)$ 7-brane in the counterclockwise direction about the branch point, the charges of the string are transformed to new charges $\left(p^{\prime \prime}, q^{\prime \prime}\right)$, as shown in Fig. 4.

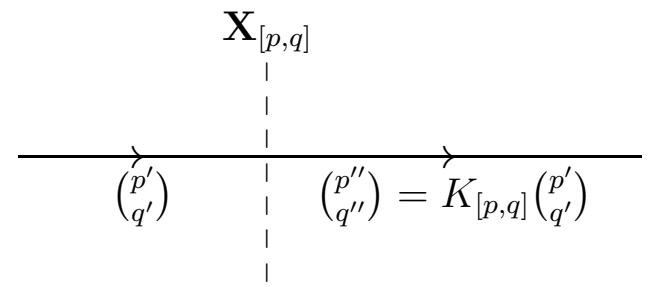

Figure 4: A string crossing a branch cut.

Let $\mathbf{z}$ denote the column vector $\left(\begin{array}{l}p \\ q\end{array}\right)$, with $\mathbf{z}^{\prime}$ and $\mathbf{z}^{\prime \prime}$ defined analogously. Then, the transformation is $\mathbf{z}^{\prime \prime}=K_{[p, q]} \mathbf{z}^{\prime}$, where $K_{[p, q]}$ is the monodromy matrix

$$
K_{[p, q]}=\left(\begin{array}{cc}
1+p q & -p^{2} \\
q^{2} & 1-p q
\end{array}\right) \in \mathrm{SL}(2, \mathbb{Z})
$$


In particular, the monodromy matrices for $\mathbf{A}, \mathbf{B}, \mathbf{C}$, and the pair $\mathbf{O}=\mathbf{B C}$ are

$$
\begin{gathered}
K_{\mathbf{A}}=\left(\begin{array}{cc}
1 & -1 \\
0 & 1
\end{array}\right), \quad K_{\mathbf{B}}=\left(\begin{array}{cc}
0 & -1 \\
1 & 2
\end{array}\right), \quad K_{\mathbf{C}}=\left(\begin{array}{cc}
2 & -1 \\
1 & 0
\end{array}\right), \\
\text { and } K_{\mathbf{O}}=K_{\mathbf{C}} K_{\mathbf{B}}=-\left(\begin{array}{ll}
1 & 4 \\
0 & 1
\end{array}\right) .
\end{gathered}
$$

The fact that $K_{\mathbf{O}}$ equals $K_{\mathbf{A}}^{-4}$ up to a minus sign indicates that an O7-plane has RR charge -4 times the charge of a D7-brane. The overall minus sign is due to the orientifold involution.

The monodromy matrices also have a topological interpretation on the K3 surface. Here, $p$ and $q$ give the components of a 1-cycle $p \alpha+q \beta$ in an elliptic fiber, and $K_{[p, q]}$ determines how these components transform when the cycle crosses the branch cut.

In the case of $\mathbb{P}^{1}$ base, a loop that encloses all 7-branes (or singular elliptic fibers) is contractible to the (smooth) point at infinity on $\mathbb{P}^{1}$. Therefore, the associated monodromy must be trivial. Indeed, for the $\mathbf{A}, \mathbf{B}, \mathbf{C}$ description of $\mathrm{K} 3$,

$$
\left(K_{\mathbf{C}} K_{\mathbf{B}}\right)^{4} K_{\mathbf{A}}{ }^{16}=1 .
$$

Finally, since the $(p, q)$ charges of strings are tranformed when crossing branch cuts, the charges of 7-branes (on which they can end) are also transformed. Thus, the collection of 7-branes corresponding to a given F-theory compactification is unique only up to: (i) braiding operations in which 7-branes are are successively transported through the branch cuts of other 7-branes and (ii) an overall $S L(2, \mathbb{Z})$ conjugation of all monodromies. ${ }^{7}$ Examples of braiding operations can be found in App. A. The collection of 7-branes (2.1) describing F-theory on $\mathrm{K} 3$ is unique up to these equivalences.

\subsection{String junctions and gauge symmetry}

Given a collection of $N$ parallel D7-branes, no two coincident, the massive $W$-bosons for the spontaneously broken $S U(N)$ worldvolume gauge symmetry are $(1,0)$ fundamental strings stretched between the $(1,0)$ D7-branes. In the presence of an O7-plane, an enhancement to $S O(2 N)$ occurs when the D7-branes are all coincident with the O7-plane. In the perturbative description, the additional $W$-bosons associated with the spontaneous breaking of $S O(2 N)$ (over those of $S U(N)$ ) are strings stretched between the D7-branes and the O7-plane. (On the covering space of the orientifold, these are strings connecting D7-branes with their $\mathbb{Z}_{2}$ images.) In all cases, the masses of the $W$-bosons can be attributed to finite string lengths $\times$ finite tensions.

\footnotetext{
${ }^{7}$ An overall $S L(2, \mathbb{Z})$ conjugation can often be achieved by braiding. In this case, the equivalence (ii) is redundent.
} 
Nonperturbatively, an O7-plane resolves to a $\mathbf{B}, \mathbf{C}$ pair of $(p, q)$-branes, so the additional $W$-bosons of $S O(2 N)$ should resolve to string junctions stretched between the D7-branes and a $\mathbf{B}, \mathbf{C}$ pair. Here, a string junction is a collection of $(p, q)$ string segments, such that each segment terminates at either a 7-brane or a vertex. At a vertex, an arbitrary number of strings can meet; the only requirement is that the total $(p, q)$ charge of the (oriented) strings entering the vertex equals the total $(p, q)$ charge leaving the vertex.

From the perturbative description, the natural guess is that an $S O(2 N) W$-boson realized as a string connecting a single D7-brane to an O7-plane should resolve to a string junction connecting a single D7 brane to a $\mathbf{B}, \mathbf{C}$ pair. But, such a junction is impossible with integer $(p, q)$ and charge conservation at the trivalent vertex. In fact, a perturbative $\mathrm{D} 7 \rightarrow \mathrm{O} 7$ string represents half of a root of $S O(2 N)$. The $\mathrm{D} 7_{i} \rightarrow \mathrm{D} 7_{i+1}$ strings span the root lattice of $S U(N)$. Here, $i=1,2, \ldots, N$ denotes an ordering of the D7-branes and an arrow denotes an oriented string stretch between the two objects. Adding the combination $\left(\mathrm{D} 7_{N-1} \rightarrow \mathrm{O} 7\right) \oplus\left(\mathrm{D} 7_{N} \rightarrow\right.$ O7) enlarges the lattice to the root lattice of $S O(2 N)$ [48]. The nonperturbative resolution of the last root is a string junction connecting two D7-branes to a $\mathbf{B}$ and $\mathbf{C}$ brane. As shown in Fig. 5, the two $(1,0)$ strings emanating from the D7 branes join to form a $(2,0)$ string; then, the $(2,0)$ string splits to form a $(1,-1)$ plus a $(1,1)$ string, which terminate on the $\mathbf{B}$ and $\mathbf{C}$ branes.

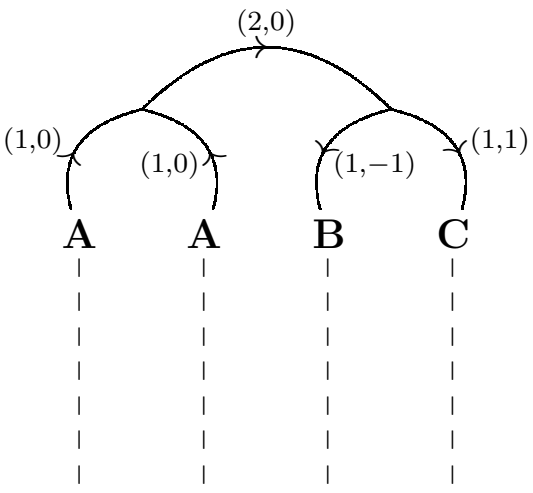

Figure 5: Massive string junction for the breaking of $S O(2 N)$ to $S U(N)$.

\subsection{Junction lattice}

The general formalism was worked out in a series of papers by DeWolfe et al. $[15,16,17,18]$. Given a collection of 7-branes, ordered as in Fig. 3, we define a lattice of equivalence classes of string junctions as follows.

First, to each string junction, we associate a lattice vector

$$
Q=\sum_{i} Q_{i} \mathbf{s}_{i},
$$


where $Q_{i} \in \mathbb{Z}$ is the net number of strings leaving (minus entering) the $i$ th 7 -brane $\mathbf{X}_{\left[p_{i}, q_{i}\right]}$, and the $\mathbf{s}_{i}$ for $i=1, \ldots, N$ are a formal basis for a rank $N$ lattice, where $N$ is the number of 7-branes. We can think of $\mathbf{s}_{i}$ as an outward oriented $\left(p_{i}, q_{i}\right)$ half-string emanating from $\mathbf{X}_{\left[p_{i}, q_{i}\right]}$. Two strings junctions are equivalent if they have the same $Q$.

Each equivalence class $Q$ can be represented by a junction in standard presentation, that is, by a tree-graph with trivalent vertices. The nontrivial step in converting a given representative to standard presentation is the operation of pushing a string through a 7-brane $\mathbf{X}_{[p, q]}$. This operation is illustrated in Fig. 6. Below the 7-brane, string charge conservation requires a discontinuity from $\mathbf{z}^{\prime}=\left(\begin{array}{c}p^{\prime} \\ q^{\prime}\end{array}\right)$ to $\mathbf{z}^{\prime \prime}=K_{[p, q]} \mathbf{z}^{\prime}$ across the branch cut. Above the 7-brane, the discontinuity can only be accounted for by the appearance of a new string that connects the 7 -brane $\mathbf{X}_{[p, q]}$ to the point of discontinuity. This is an example of the Hanany-Witten effect $[32]$.

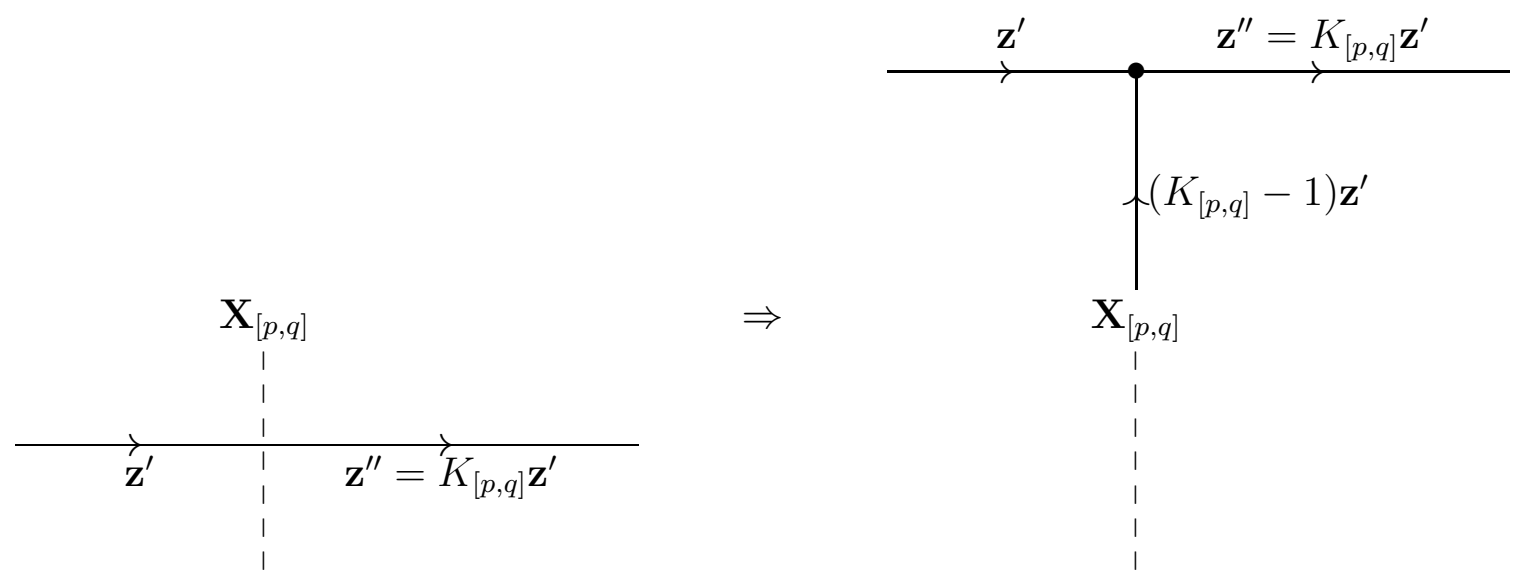

Figure 6: Pushing a string through a 7-brane: the Hanany-Witten effect.

As required, the new string emanating from $\mathbf{X}_{[p, q]}$ has string charge proportional to $\mathbf{z}=\left(\begin{array}{l}p \\ q\end{array}\right)$ :

$$
\left(K_{[p, q]}-1\right) \mathbf{z}^{\prime}=\left(\mathbf{z}^{\prime} \cdot \mathbf{z}\right)\left(\begin{array}{l}
p \\
q
\end{array}\right), \quad \text { where } \quad \mathbf{z}^{\prime} \cdot \mathbf{z}=\left|\begin{array}{ll}
p^{\prime} & p \\
q^{\prime} & q
\end{array}\right|=p^{\prime} q-q^{\prime} p
$$

The junction lattice $J$ is defined to be the lattice of equivalence classes $Q$ of junctions together with the inner product,

$$
\begin{aligned}
& \left(s_{i}, s_{i}\right)=-1, \\
& \left(s_{i}, s_{j}\right)=\left(s_{j}, s_{i}\right)=\frac{1}{2}\left(p_{i} q_{j}-p_{j} q_{i}\right), \quad i<j .
\end{aligned}
$$

A good way to think about this inner product and the tranformation rule (2.8) is that they are both related to the antisymmetric intersection pairing $\left(p^{\prime}, q^{\prime}\right) \cdot(p, q)=p^{\prime} q-q^{\prime} p$ 
of 1-cycles on the elliptic fiber. To define the symmetric inner product (2.9), we use the additional structure provided by the ordering of the branch cuts.

On $\mathbb{C}^{1}$ or an open subset of $\mathbb{P}^{1}$, the junction lattice $J$ is the full rank $N$ lattice generated by the $\mathbf{s}_{i}$. However, some of the lattice vectors correspond to junctions with asymptotic $(p, q)$ charge at infinity. We define the proper junction lattice $J_{\text {proper }}$ to be the sublattice of proper string junctions in which no net charge is carried by strings that run off to infinity, ${ }^{8}$

$$
\sum_{i} Q_{i}\left(\begin{array}{l}
p_{i} \\
q_{i}
\end{array}\right)=\left(\begin{array}{l}
0 \\
0
\end{array}\right) \quad \text { (proper junction lattice). }
$$

This constraint means that the rank of $J_{\text {proper }}$ is less than the number of 7 -branes by 1 or $2 .{ }^{9}$

On $\mathbb{P}^{1}$, there is no distinction between the junction lattice and proper junction lattice. The point at infinity is contained in $\mathbb{P}^{1}$, and a string cannot terminate there unless it is the location of a 7 -brane or vertex. Thus, on $\mathbb{P}^{1}$, we have $J=J_{\text {proper }}$, of rank less than the number of 7 -branes $N$.

The root lattices of the A-D-E Lie algebras can each be realized as the proper junction lattice of collections of $\mathbf{A}, \mathbf{B}$ and $\mathbf{C}$ type 7-branes, as indicated in Table 1. (See Refs. [15, $16,17,18]$ for further details.)

$$
\begin{array}{ll}
A_{N} & \mathbf{A}^{N+1} \quad(N \geq 1), \\
D_{N} & \mathbf{A}^{N} \mathbf{B C} \quad(N \geq 4), \\
E_{N} & \mathbf{A}^{N-1} \mathbf{B C C} \quad(N=6,7,8), \\
H_{N} & \mathbf{A}^{N+1} \mathbf{C} \quad(N=0,1,2) .
\end{array}
$$

Table 1: A-D-E Lie algebras and the corresponding 7-brane collections.

The proper string junctions of each of these collections are the $W$-bosons of the corresponding gauge symmetry. When the 7 -branes coalesce to a point, the $W$-bosons are massless and the gauge symmetry is unbroken. In general, a collection of 7-branes can coalesce if and only if the inner product of the proper junction lattice of the collection is negative definite. This condition is satisfied for the classical A-D-E Lie algebras with $N$ in the ranges given in Table 1, but not for the more exotic algebras like $E_{N}$ with $N>8$.

The $H_{N}$ row of Table 1 provides a second way to realize $A_{N}$ gauge symmetry. In contrast to the perturbative realization via $N+1 \mathrm{~A}$ branes (D7-branes), the $H_{N}$ realization is strongly coupled. Likewise, in the moduli space of the $\mathcal{N}=2$ worldvolume theory on a D3-brane probe

\footnotetext{
${ }^{8}$ Eq. (2.10) gives a homomophism from $\operatorname{Span}\left(\left\{\mathbf{s}_{i}\right\}\right) \cong \mathbb{Z}^{N}$ to the $\mathbb{Z}^{2}$ of $(p, q)$ string charges. The proper junction lattice is the kernel of this homomorphism.

${ }^{9}$ For example, for a collection of D7 branes only, Eq. (2.10) gives only 1 constraint since $q_{i}=0$ for all $i$. For a collection that spans the $\mathbb{Z}^{2}$ of possible $(p, q)$, there are 2 constraints.
} 
in the presence of a coalesced $H_{N}$ collection of 7-branes, there is a strongly coupled ArgyresDouglas point [1], at which $N+1$ hypermultiplets (of two mutually nonlocal electromagnetic charges) become massless. The hypermultiplets come from a $(1,0)$ string or $(1,1)$ string stretched between the D3 brane and an $\mathbf{A}$ or $\mathbf{C}$ brane, respectively.

In terms of the elliptic fibration, string junctions lift to 2-cycles in $\mathcal{X}$. A junction in standard presentation (i.e., a tree graph) lifts to a 2-cycle that is topologically $S^{2}$. As mentioned earlier, the 2-cycle is obtained by fibering the circle $S_{p, q}^{1} \subset T^{2}$ over each $(p, q)$ string segment in $\mathbb{P}^{1}$ (see Fig. 7). The 2-cycle smoothly pinches off at the locations of the singular fibers, at which an $S^{1}$ shrinks to zero size. The junction inner product (2.9) reproduces the standard intersection pairing on $H_{2}(\mathcal{X})$. The statement that a collection of 7-branes can coalesce to a point on $\mathbb{P}^{1}$ only for negative definite inner product reproduces the standard result that a collection of 2-cycles can be collapse to zero size only for negative definite intersection matrix.

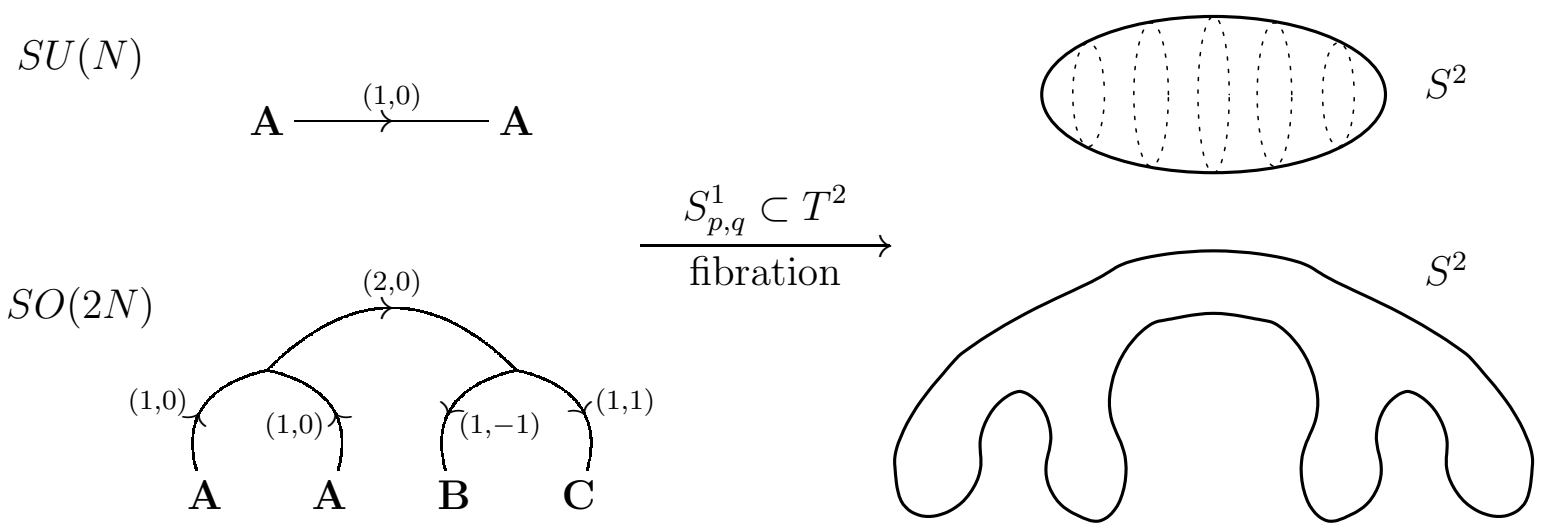

Figure 7: Lift of $S U(N)$ roots and the additional roots of $S O(2 N)$ from string junctions stretched between 7 -branes in type IIB to 2 -cycles in $\mathcal{X}$.

Finally, coalescing $(p, q)$ 7-branes in type IIB correspond to coalescing $\mathrm{I}_{1}$ singular fibers in $\mathcal{X}$. The relation between the choice of coalescing collection (in terms of $\mathbf{A}, \mathbf{B}$ and $\mathbf{C}$ type $\mathrm{I}_{1}$ fibers) and the Kodaira type of the resulting singular fiber is shown in Table $2[57,5]$. The table also indicates the number of irreducible components of the resulting singular fiber and the intersection matrix of these components.

\subsubsection{Junction lattice of $\frac{1}{2} \mathrm{~K} 3=\mathrm{dP}_{9}$}

The simplest nontrivial choice of the Calabi-Yau manifold $\mathcal{X}$ is an elliptic K3 surface. In this case, we can simplify matters further by considering the stable degeneration limit, in which the base degenerates to two $\mathbb{P}^{1} \mathrm{~s}$ meeting at a point, and $\mathrm{K} 3$ factorizes into two $\mathrm{dP}_{9}$ 
Coalescing collection Kod. type Number of components

$\begin{array}{lllll}- & - & \mathrm{I}_{0} & 1 \text { (elliptic) } & 0 \\ A_{N-1} & \left(\mathbf{A}^{N}\right) & \mathrm{I}_{N} & N(N \text { distinct intersect. pts. }) & \text { affine } A_{N-1} \\ D_{N+4} & \left(\mathbf{A}^{N+4} \mathbf{B C}\right) & \mathrm{I}_{N}^{*} & N+5 & \text { affine } D_{N+4} \\ E_{6} & \left(\mathbf{A}^{5} \mathbf{B C C}\right) & \mathrm{IV}^{*} & 7 & \text { affine } E_{6} \\ E_{7} & \left(\mathbf{A}^{6} \mathbf{B C C}\right) & \mathrm{III}^{*} & 8 & \text { affine } E_{7} \\ E_{8} & \left(\mathbf{A}^{7} \mathbf{B C C}\right) & \mathrm{II}^{*} & 9 & \text { affine } E_{8} \\ H_{0} & (\mathbf{A C}) & \text { II } & 1 \text { (with cusp) } & 0 \\ H_{1} & \left(\mathbf{A}^{2} \mathbf{C}\right) & \text { III } & 2 \text { (meet in one pt. of order 2) } & \text { affine } A_{1} \\ H_{2} & \left(\mathbf{A}^{3} \mathbf{C}\right) & \text { IV } & 3 \text { (all meet in 1 pt.) } & \text { affine } A_{2}\end{array}$

Intersection matrix

Table 2: Coalescing collections of $\mathrm{I}_{1}$ fibers and Kodaira type of the resulting singular fiber.

surfaces ${ }^{10}$ meeting in an elliptic curve. Via duality to the $E_{8} \times E_{8}$ heterotic string, each $\mathrm{dP}_{9}$ corresponds to a single $E_{8}$ factor, so we can focus on a single $\mathrm{dP}_{9}$.

As an elliptic fibration, a generic smooth $\mathrm{dP}_{9}$ has 12 singular fibers of type $\mathrm{I}_{1}$. Up to the equivalences discussed in App. A (braiding and $S L(2, \mathbb{Z})$ conjugation), the collection of singular fibers is $\mathbf{A}^{8} \mathbf{B C B C}$ - exactly half of the corresponding collection (2.1) for K3. The total monodromy about all singular fibers is again unity, as required,

$$
\left(K_{\mathbf{C}} K_{\mathbf{B}}\right)^{2} K_{\mathbf{A}}^{8}=1 .
$$

The junction lattice of $\mathrm{dP}_{9}$ was analyzed in great detail in Ref. [23]. For a smooth $\mathrm{dP}_{9}$ with $12 \mathrm{I}_{1}$ fibers, the junction lattice is the semidefinite lattice

$$
J=E_{8}^{-} \oplus \mathbb{Z} \boldsymbol{\delta}_{1} \oplus \mathbb{Z} \boldsymbol{\delta}_{2}
$$

the direct sum of the $E_{8}^{-}$lattice (where the minus indicates that the inner product is minus that of the $E_{8}$ root lattice) and a 2D null lattice generated by the charge vectors

$$
\begin{aligned}
& \boldsymbol{\delta}_{1}=(0,0,0,0,0,0,0,0,-1,-1,1,1), \\
& \boldsymbol{\delta}_{2}=(-1,-1,-1,-1,-1,-1,-1,-1,7,5,-3,-1) .
\end{aligned}
$$

By brane motions (the braiding operations of App. A), $\mathbf{A}^{8} \mathbf{B C B C} \cong \mathbf{A}^{7} \mathbf{B C C X} \mathbf{X}_{[3,1]} \mathbf{A}$. The $E_{8}^{-}$is then generated by the proper junctions of $\mathbf{A}^{7} \mathbf{B C C}$. The null vectors $\boldsymbol{\delta}_{1}$ and $\boldsymbol{\delta}_{2}$ have the following interpretation. They can each be represented by a loop junction, a counterclockwise loop of $(p, q)$ strings circling all of the the 7-branes. (See Fig. 8 below.) Since the total

\footnotetext{
${ }^{10} \mathrm{~A} \mathrm{dP}_{9}$ is a rational elliptic surface: rational, since it is the blow-up of $\mathbb{P}^{2}$ in nine points, and elliptic, since it admits an elliptic fibration over $\mathbb{P}^{1}$. The sections are the nine blow-up $\mathbb{P}^{1} \mathrm{~s}$ and the elliptic fiber is represented by the canonical class $K=-3 H+\sum_{i=1}^{9} \mathcal{E}_{i}$, with $K^{2}=0$. Here, $H$ is the hyperplane class of $\mathbb{P}^{2}$ and $\mathcal{E}_{i}$ is the class of the $i$ th exceptional $\mathbb{P}^{1}$.
} 
monodromy is unity, a $(p, q)$ string starting above all of the branch cuts comes back again to a $(p, q)$ string after traversing the complete loop. So, it can close. Such a loop is contractible to the point at infinity and is in this sense trivial. ${ }^{11}$ For $(p, q)=(1,0)$ and $(0,1)$, we obtain the charge vectors $\boldsymbol{\delta}_{1}$ and $\boldsymbol{\delta}_{2}$, respectively. In order to obtain the charge vectors quoted in Eq. (2.13), it is necessary to convert the loop junction to standard tree presentation by "pushing strings through vertices" using the Hanany-Witten effect, as described in Sec. 2.5.

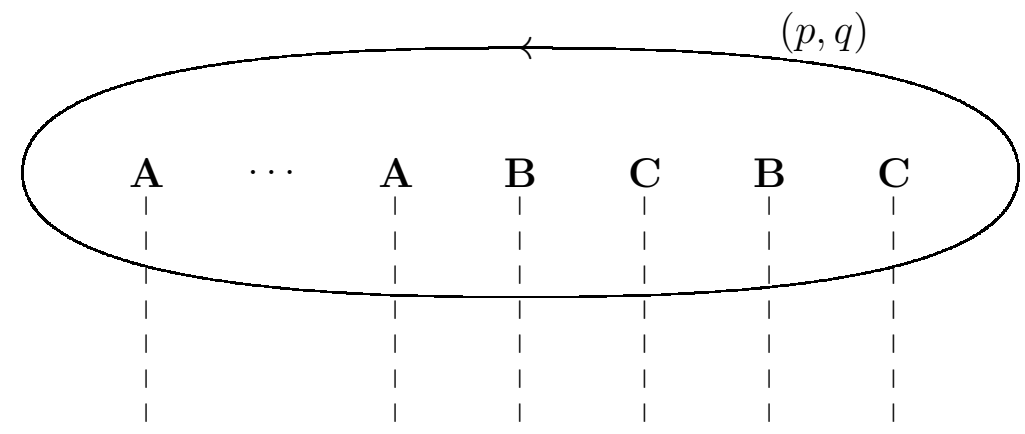

Figure 8: A loop junction, contractible to the point at infinity. The null generators $\boldsymbol{\delta}_{1}$ and $\boldsymbol{\delta}_{2}$ are obtained for $(p, q)=(1,0)$ and $(0,1)$, respectively.

\subsubsection{Mathematical interpretation of the junction lattice}

In this section, we establish terminology for variety of lattices related to the junction lattice $J$, and then relate these lattices to the homology of the elliptic fibration. (See Eqs. (2.7) and (2.9) for the definition of the junction lattice.)

1. The null junction lattice $J_{\text {null }} \subset J$ is the null sublattice of the junction lattice.

2. The loop junction lattice $J_{\text {loop }} \subset J_{\text {null }}$ is the null sublattice generated by loop junctions circling all 7-brane locations (locations of singular fibers) on $\mathbb{P}^{1}$ and contractible to the point at infinity. ${ }^{12}$

3. The effective junction lattice is the quotient

$$
J_{\text {eff }}=J / J_{\text {loop }}
$$

We have just seen that the effective junction lattice of a smooth $\mathrm{dP}_{9}$ is $J_{\text {eff }}\left(\mathrm{dP}_{9}\right)=E_{8}^{-}$.

\footnotetext{
${ }^{11}$ Likewise, the 2-cycle in $\mathcal{X}$ obtained by fibering an $S^{1}$ over this loop is a homologically trivial $T^{2}$ in $\mathcal{X}$.

${ }^{12}$ For a compact elliptic surface, there is no torsion in the Néron-Severi lattice (the algebraic part of $H_{2}(\mathcal{X}, \mathbb{Z})$ ), and no distinction between $J_{\text {loop }}$ and $J_{\text {null }}$. However, it is useful to introduce the extra terminology here, so that it will carry over to abelian surface fibrations without alteration. As we will see, $J_{\text {loop }} \neq J_{\text {null }}$ for the abelian surface fibrations $\mathcal{X}_{m, n}$ with $m \neq 1$ discussed in Sec. 3 .
} 
4. The Kodaira junction lattice $J_{\text {Kodaira }}$ is the sum of the proper junction sublattices associated to each of the collections of coalescing 7-branes. It is trivial for a smooth surface, where all singular fibers are of type $\mathrm{I}_{1}$, but nontrivial when a collection of 7-branes has coalesced and the elliptic fibration acquires a multicomponent Kodaira fiber, as in Table 2.

5. The tadpole junction lattice is

$$
J_{0}=J_{\text {Kodaira }}^{\perp} \equiv \text { orthogonal complement of } J_{\text {Kodaira }} \text { in } J_{\text {eff }} .
$$

For a smooth surface, $J_{0}=J_{\text {eff }}$. The reason for the terminology is that the sublattice orthogonal to the proper junctions associated to a subcollection of coalescing branes can be represented by junctions that are tree graphs away from the collection, with a possible termination in a tadpole loop circling the collection (cf. Figs. 9 and 10).

In Sec. 2.6, we will also define weakly integral analogs of these lattices.

Let us describe the homological interpretation of the various lattices just defined. First consider an arbitrary elliptic fibration $\pi: \mathcal{X} \rightarrow \mathcal{B}$. The existence of the projection $\pi$ induces a filtration on the cohomology that allows us to write $[2,28] .{ }^{13}$

$$
H^{2}(\mathcal{X}, \mathbb{Z})=H^{2}(\mathcal{B}, \mathbb{Z}) \oplus H^{1}\left(\mathcal{B}, R^{1} \pi_{*} \mathbb{Z}\right) \oplus H^{0}\left(\mathcal{B}, R^{2} \pi_{*} \mathbb{Z}\right)
$$

Here, $H^{p}\left(\mathcal{B}, R^{q} \pi_{*} \mathbb{Z}\right)$ can roughly be thought of as the cohomology of degree $p$ along the base and $q$ along the fiber. ${ }^{14}$ We have contributions:

1. $H^{2}(\mathcal{B}, \mathbb{Z})$ from the base,

2. $H^{0}\left(\mathcal{B}, R^{2} \pi_{*} \mathbb{Z}\right)$ from the generic fiber and extra components of reducible fibers,

3. $H^{1}\left(\mathcal{B}, R^{1} \pi_{*} \mathbb{Z}\right)$ from everything else.

Now restrict to an elliptic surface $\mathcal{X}$. The string junctions of the previous section are real $1 \mathrm{D}$ graphs on $\mathcal{B}$ that lift to 2 -cycles in $\mathcal{X}$ by fibering an $S^{1} \subset T^{2}$ over each segment of the graph. Thus we might expect that they are related to $H^{1}\left(\mathcal{B}, R^{1} \pi_{*} \mathbb{Z}\right)$. Indeed, this is the interpretation of the tadpole junction lattice given in Ref. [23],

$$
J_{0}=H^{1}\left(\mathcal{B}, R^{1} \pi_{*} \mathbb{Z}\right)
$$

\footnotetext{
${ }^{13}$ To be precise, Eq. (2.16) is not literally correct as written. There exists a filtration, but additional assumptions are necessary for the sequence to split as shown. For a further discussion, see App. H.

${ }^{14}$ The sheaf $R^{q} \pi_{*} \mathbb{Z}$ on $\mathcal{B}$ associates the group $H^{q}\left(\pi^{-1}(U), \mathbb{Z}\right)$ to each open set $U \subset \mathcal{B}$. For $U$ a neighborhood of a generic point $x \in \mathbb{P}^{1}$ this becomes the cohomology group $H^{q}\left(f_{x}, \mathbb{Z}\right)$ along the elliptic fiber $f_{x}=\pi^{-1}(x)$, modulo monodromy equivalences. See App. $\mathrm{H}$ for further background on $R^{q} \pi_{*}$.
} 
Likewise,

$$
H^{0}\left(\mathcal{B}, R^{2} \pi_{*} \mathbb{Z}\right)=J_{\text {Kodaira }} \oplus F \mathbb{Z}
$$

where $F$ is the generic fiber.

The group of rational sections of an elliptic fibration is known as the Mordell-Weil group MW. The narrow Mordell-Weil group $\mathrm{MW}_{0}$ is the subgroup of sections that intersect the same components of singular fibers as the zero section $\sigma_{0}$. In general $[13,23,2]$,

$$
\mathrm{MW}_{0}(\mathcal{X})=H^{1}\left(\mathcal{B}, R^{1} \pi_{*} \mathbb{Z}\right) \cap H^{1,1}(\mathcal{X}, \mathbb{C}) .
$$

For $\mathrm{dP}_{9}$, the intersection removes nothing, and $\mathrm{MW}_{0}=H^{1}\left(\mathcal{B}, R^{1} \pi_{*} \mathbb{Z}\right)=J_{0}$, where $J_{0}=E_{8}^{-}$ from the previous section. This is the situation to keep in mind for the generalization to abelian surface fibered Calabi-Yau manifolds in Sec. 3, where again $H^{2,0}(\mathcal{X})=0$. On the other hand, for $\mathcal{X}$ an elliptic $\mathrm{K} 3, H^{2,0} \neq 0$, and the intersection with $H^{1,1}$ depends on the choice of complex structure moduli. ${ }^{15}$

The junction description of the full Mordell-Weil group MW and its torsion subgroup $M W_{\text {tor }}$ will be given in Sec. 2.6, once we have introduced the notion of weak integrality.

\subsubsection{An example with coalesced 7-branes}

We now turn to an example in which some of the 7-branes ( $I_{1}$ fibers of $\mathcal{X}$ ) have coalesced. For simplicity, we start again with $\mathrm{dP}_{9}$. From the braiding operations discussed in App. A, we have

$$
\mathbf{A}^{8} \mathbf{B C B C} \cong \mathrm{A}^{4} \mathbf{B C A}^{2} \mathbf{B C A}^{2} \cong \mathbf{A}^{4} \mathbf{B A}^{2} \mathbf{B} \mathbf{B A}^{2} \mathbf{B} \cong \mathbf{A}^{4} \mathbf{B}^{2} \mathbf{D}^{2} \mathbf{B}^{2} \mathbf{D}^{2}
$$

where $\mathbf{D}=\mathbf{X}_{[0,1]}$. We adopt the last collection $\mathbf{A}^{4} \mathbf{B}^{2} \mathbf{D}^{2} \mathbf{B}^{2} \mathbf{D}^{2}$ for this example.

In the basis corresponding to this collection, the null loop junctions of Sec. 2.5 become

$$
\begin{aligned}
& \boldsymbol{\delta}_{1}=(0,0,0,0,-1,-1,-1,-1,1,1,1,1), \\
& \boldsymbol{\delta}_{2}=(-1,-1,-1,-1,3,3,-2,-2,-1,-1,0,0),
\end{aligned}
$$

from loops with $(p, q)=(1,0)$ and $(0,1)$, respectively. In our collection, adjacent branes of the same type can coalesce. We will use surrounding parentheses to denote subcollections of coalesced branes. Thus,

$$
\left(\mathbf{A}^{2}\right)\left(\mathbf{A}^{2}\right)\left(\mathbf{B}^{2}\right)\left(\mathbf{D}^{2}\right)\left(\mathbf{B}^{2}\right)\left(\mathbf{D}^{2}\right), \quad \text { with } \quad J_{\text {Kodaira }}=A_{1}^{-\oplus 6},
$$

denotes a brane collection in which all branes have coalesced pairwise. In the elliptic fibration of the $\mathrm{dP}_{9}$, the twelve $\mathrm{I}_{1}$ fibers have coalesced pairwise to become six $\mathrm{I}_{2}$ fibers, each giving

\footnotetext{
${ }^{15}$ For an elliptic $\mathrm{K} 3, H^{1}\left(\mathcal{B}, R^{1} \pi_{*} \mathbb{Z}\right)=E_{10}^{-} \oplus E_{10}^{-}$is a signature $(2,18)$ sublattice of $H^{2}(\mathrm{~K} 3, \mathbb{Z})$, which when combined with $\sigma_{0}, f$ gives the full $H^{2}$ lattice [18]. On the other hand $\mathrm{MW}_{0}$ varies from rank 0 to 16 depending on complex structure.
} 
an $A_{1}$ surface singularity. The Kodaira junction lattice is $A_{1}^{-\oplus 6}$, generated by $(p, q)$ strings that connect the two 7-branes in each pair:

$$
\begin{aligned}
& \boldsymbol{\alpha}_{1}=\mathbf{s}_{1}-\mathbf{s}_{2}, \quad \boldsymbol{\alpha}_{2}=\mathbf{s}_{3}-\mathbf{s}_{4}, \quad \boldsymbol{\alpha}_{3}=\mathbf{s}_{5}-\mathbf{s}_{6}, \\
& \boldsymbol{\alpha}_{4}=\mathbf{s}_{7}-\mathbf{s}_{8}, \quad \boldsymbol{\alpha}_{5}=\mathbf{s}_{9}-\mathbf{s}_{10}, \quad \boldsymbol{\alpha}_{6}=\mathbf{s}_{11}-\mathbf{s}_{12} .
\end{aligned}
$$

Here, $A_{1}^{-}$denotes a lattice whose inner product is minus that of the $A_{1}$ root lattice.

The tadpole junction lattice $J_{0}=J_{\text {Kodaira }}^{\perp} \subset J_{\text {eff }}$ is generated by tadpole junctions modulo null loops. Recall that tadpole junctions terminate at noncoalesced branes and/or at tadpole loops circling coalesced branes. ${ }^{16}$ It is meaningful to quotient by null loops since the null junctions (2.21) can also be represented by tadpole junctions. For example, $\boldsymbol{\delta}_{2}$ is shown in Fig. 9. Tadpole junctions representing the lattice vectors

$$
\begin{aligned}
& \boldsymbol{\beta}_{1}=\mathbf{s}_{1}+\mathbf{s}_{2}-\mathbf{s}_{3}-\mathbf{s}_{4}, \\
& \boldsymbol{\beta}_{2}=\mathbf{s}_{5}+\mathbf{s}_{6}-\mathbf{s}_{9}-\mathbf{s}_{10},
\end{aligned}
$$

are shown in Fig. 10. Together, $\boldsymbol{\beta}_{1}$ and $\boldsymbol{\beta}_{2}$ span the tadpole junction lattice of the collection $(2.22):{ }^{17}$

$$
J_{0}=\boldsymbol{\beta}_{1} \mathbb{Z} \oplus \boldsymbol{\beta}_{2} \mathbb{Z}=A_{1}^{-\oplus 2}
$$

This lattice is isomorphic to the narrow Mordell-Weil lattice $\mathrm{MW}_{0}$ of the corresponding singular elliptic $\mathrm{dP}_{9}$ with six $\mathrm{I}_{2}$ fibers.

\subsection{Weakly integral junction lattice and torsion sections}

In the presence of coalesced branes, there exists a natural extension of the junction lattice $J$ known as the weakly integral junction lattice $J^{\text {weak }}$ [23]. So far we have considered only physical string junctions in which the string charges $(p, q)$ of each segment are integral. A weakly integral string junction is a string junction in which we relax the integrality requirement on the strings in tadpole loops, requiring only that the string charge entering or leaving a tadpole termination be integral. For example, consider a $\mathrm{dP}_{9}$ surface. From the junctions in Figs. 9 and 10, we obtain the weakly integral junctions $\boldsymbol{\delta}_{2} / 2, \boldsymbol{\beta}_{1} / 2$, and $\boldsymbol{\beta}_{2} / 2$ shown in Figs. 11 and 12 .

\footnotetext{
${ }^{16}$ Instead of the tadpole terminations, we can alternatively push the tadpole loops through branch points to obtain terminations at coalesced branes. Note however, that in the full junction lattice, the charge vector of the resulting junction diagram is ambiguous. For example, a $(2,0)$ string terminating on a coalesced $\left(\mathbf{A}^{2}\right)$ pair might have the junction charges of a $(1,0)$ string terminating on each $\mathbf{A}$, or a $(2,0)$ string terminating on one of them. A tadpole termination makes it unambigous that we mean the former (by a small deformation of the coalesced brane locations). This ambiguity disappears once we restrict to the tadpole junction lattice, where the only charges permitted are those corresponding to the tadpole terminations.

${ }^{17}$ A similar junction joining the two $\left(\mathbf{D}^{2}\right)$ 's in Fig. 10 differs from $\boldsymbol{\beta}_{2}$ by $\boldsymbol{\delta}_{1}$, so it is not linearly independent.
} 


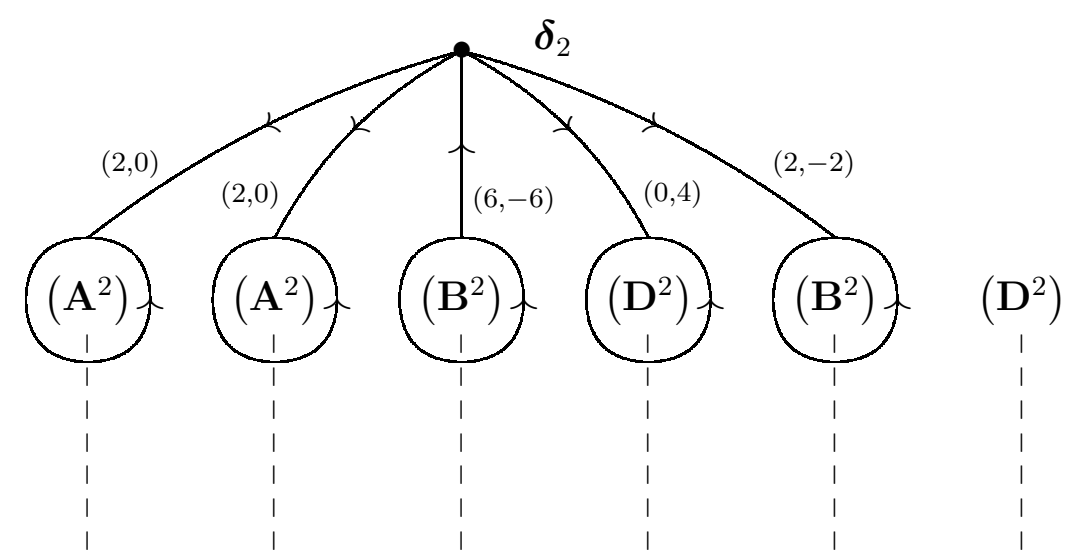

Figure 9: The null junction $\boldsymbol{\delta}_{2}$ represented as a tadpole junction.
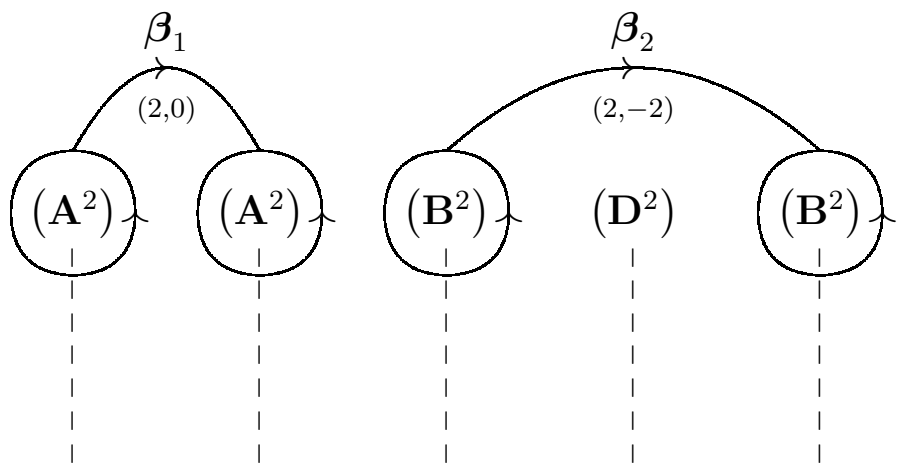

$\left(\mathrm{D}^{2}\right)$

Figure 10: Tadpole junctions $\boldsymbol{\beta}_{1}$ and $\boldsymbol{\beta}_{2}$.

Now return to the case of a general elliptic surface. In addition to $J^{\text {weak }}$, we define the related junction lattices $J_{\text {null }}^{\text {weak }}, J_{\text {eff }}^{\text {weak }}, J_{\text {Kodaira }}^{\text {weak }}$ and $J_{0}^{\text {weak }}$. The definitions are straightforward analogs of those in Sec. 2.5.2, with the possible exception of loop junction sublattice, which is defined to be the same in either case: $J_{\text {loop }}^{\text {weak }}=J_{\text {loop }}$.

The full Mordell-Weil group and its torsion subgroup are determined by the various junction lattices as follows [23]:

$$
\begin{aligned}
\mathrm{MW} & =J_{0}^{\text {weak }} & & \text { (weakly integral tadpole junctions mod loops) }, \\
\mathrm{MW}_{\text {tor }} & =J_{\text {null }}^{\text {weak }} / J_{\text {loop }} & & (\text { weakly integral null junctions mod loops) } .
\end{aligned}
$$

When the Néron-Severi lattice ${ }^{18} \operatorname{NS}(\mathcal{X})$ of the elliptic surface is unimodular, the MordellWeil lattice $\mathrm{MW} / \mathrm{MW}_{\text {tor }}$ is $M W_{0}^{*}=J_{0}^{*}$, the dual lattice of the narrow Mordell-Weil lattice and tadpole junction lattice. In particular, this is the case for $\mathrm{dP}_{9}$.

\footnotetext{
${ }^{18}$ The Néron-Severi lattice is the lattice of algebraic divisors modulo algebraic equivalence. For a compact elliptic surface $\mathcal{X}$, the Néron-Severi lattice is torsion free; under the isomorphism $H_{2}(\mathcal{X}, \mathbb{Z}) \cong H^{2}(\mathcal{X}, \mathbb{Z})$, it is the sublattice of $H_{2}(\mathcal{X}, \mathbb{Z})$ identified with $H^{2}(\mathcal{X}, \mathbb{Z}) \cap H^{1,1}(\mathcal{X}, \mathbb{C})$.
} 


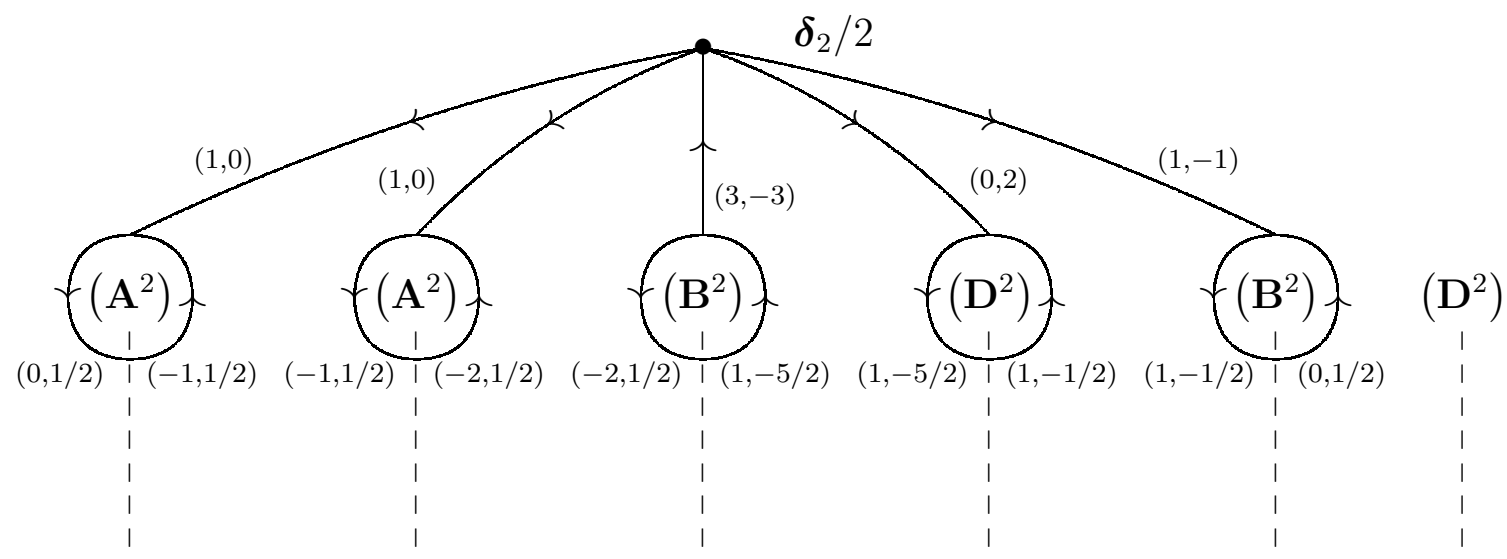

Figure 11: The weakly integral null junction $\boldsymbol{\delta}_{2} / 2$.
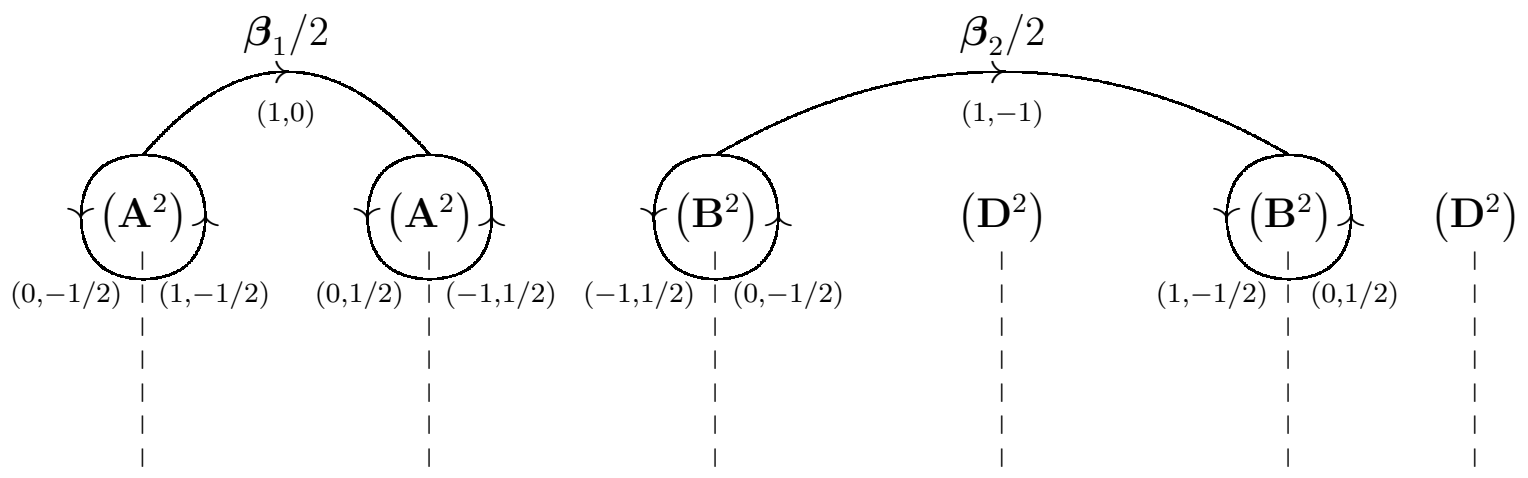

Figure 12: Weakly integral tadpole junctions $\boldsymbol{\beta}_{1} / 2$ and $\boldsymbol{\beta}_{2} / 2$.

In the singular $\mathrm{dP}_{9}$ example above, $J_{0}=A_{1}{ }^{\oplus 2}$, so $\mathrm{MW} / \mathrm{MW}_{\text {tor }}=A_{1}^{*}{ }^{\oplus 2}$. The weakly integral null lattice is

$$
J_{\text {null }}^{\text {weak }}=\left(\boldsymbol{\delta}_{1} / 2\right) \mathbb{Z} \oplus\left(\boldsymbol{\delta}_{2} / 2\right) \mathbb{Z},
$$

so $\mathrm{MW}_{\text {tor }}=J_{\text {null }}^{\text {weak }} / J_{\text {loop }}=\mathbb{Z}_{2} \oplus \mathbb{Z}_{2}$, generated by $\boldsymbol{\delta}_{1} / 2$ and $\boldsymbol{\delta}_{2} / 2$.

For a more complete and very readable exposition on the relation between string junctions and the Mordell-Weil lattice, the reader is referred to Ref. [23], in which the Oguiso-Shioda classification of the Mordell-Weil lattices of $\mathrm{dP}_{9}$ is reproduced entirely in the junction framework. ${ }^{19}$ As emphasized in Ref. [23], $\mathrm{MW}_{\text {tor }}$ has physical consequences. Whereas the gauge algebra on a collection of coalescing 7-branes is determined by its root lattice, and hence by $J_{\text {Kodaira }}$, the group $\mathrm{MW}_{\text {tor }}$ determines $\pi_{1}$ of the global gauge group.

\footnotetext{
${ }^{19}$ Indeed, Ref. [23] even caught a two errors in Oguiso and Shioda's list of Mordell-Weil groups of dP 9 [40].
} 


\section{Monodromy and junction description of CY duals of $T^{6} / \mathbb{Z}_{2}$}

\subsection{The duality map}

In Ref. [46], it was shown that type IIB $T^{6} / \mathbb{Z}_{2}$ orientifold with a choice of flux preserving $\mathcal{N}=2$ supersymmetry is dual to a class of purely geometrical type IIA Calabi-Yau compactifications with no flux. The dual Calabi-Yau manifolds $\mathcal{X}_{m, n}$ are abelian surface fibrations over $\mathbb{P}^{1}$. They bear a number of similarities to the elliptic fibrations over $\mathbb{P}^{1}$ described in Sec. 2. In this section, we will provide a similar monodromy and junction based description of the topology of $\mathcal{X}_{m, n}$ and its geometry of curves.

To see why the IIA dual geometry is a $T^{4}$ fibration, first recall that in the absence of flux, we have the $\mathcal{N}=4$ duality,

$$
\begin{aligned}
T^{6} / \mathbb{Z}_{2} \text { orientifold } \leftrightarrow \quad & \text { IIA on } \mathrm{K} 3 \times T^{2} \\
& \left(\text { where } \mathrm{K} 3=T^{2} \text { fibration over } P^{1}\right) .
\end{aligned}
$$

Here, note that $\mathrm{K} 3 \times T^{2}$ can be viewed as $T^{4}$ fibration over $\mathbb{P}^{1}$, where a $T^{2} \subset T^{4}$ trivially factorizes. This duality can be understood in several ways. Two are as follows.

1. Via heterotic/IIA duality: The IIB $T^{6} / \mathbb{Z}_{2}$ orientifold $\leftrightarrow$ type I on $T^{6}$ (by T-duality) $\leftrightarrow$ heterotic $S O(32)$ on $T^{6}$ (by S-duality) $\leftrightarrow$ heterotic $E_{8} \times E_{8}$ on $T^{6}$ (by T-duality) $\leftrightarrow$ IIA on $\mathrm{K} 3 \times T^{2}$ (by heterotic/IIA duality).

2. Via M-theory: First T-dualize $T^{6} / \mathbb{Z}_{2}$ on a $T^{3}$ to obtain the IIA $T^{3} / \mathbb{Z}_{2} \times T^{3}$ D6/O6 orientifold. This is dual to type IIA on $\mathrm{K} 3 \times T^{2}$ via a circle swap: The IIA orientifold lifts to M-theory on $\mathrm{K} 3 \times T^{3}$, where the $\mathrm{K} 3 \cong T^{4} / \mathbb{Z}_{2}$ comes from the lift of the $T^{3} / \mathbb{Z}_{2}$ factor. Then compactifying on an $S^{1}$ in the $T^{3}$ factor gives IIA on $\mathrm{K} 3 \times T^{2}$.

The modification of the duality (3.1) due to $\mathcal{N}=2$ flux is as follows:

$$
\begin{aligned}
T^{6} / \mathbb{Z}_{2} \text { orientifold } \leftrightarrow \quad & \text { IIA on a Calabi-Yau } \mathcal{X}_{m, n} \\
& \left(\text { where } \mathcal{X}_{m, n}=T^{4} \text { fibration over } P^{1}\right) .
\end{aligned}
$$

The flux dualizes to twists of the topology that: (i) mix the previous $T^{2}$ factor with the $T^{2}$ fiber of K3, and (ii) require a reduction in the number of exceptional divisors from the 16 that would be associated with $\mathrm{K} 3$ to a smaller number $M<16$. On the $T^{6} / \mathbb{Z}_{2}$ side of the duality, the integers $m$ and $n$ parametrize the choice of flux

$$
\begin{aligned}
& F_{\mathrm{RR}}=2 m\left(d x^{1} \wedge d y^{1}+d x^{2} \wedge d y^{2}\right) \wedge d y^{3} \\
& H_{\mathrm{NS}}=2 n\left(d x^{1} \wedge d y^{1}+d x^{2} \wedge d y^{2}\right) \wedge d x^{3}
\end{aligned}
$$

and $M$ is the number of D3-branes. 
In Ref. [46], this duality was studied by mapping the family of classical 10D type IIB supergravity solutions through the duality chain 2 above. The resulting description of $\mathcal{X}_{m, n}$ includes an explicit metric that is valid to leading order in the relative Kähler modulus $h / s$ (fiber size/base size). The harmonic forms in the metric can also be given explicitly. For $h / s \ll 1$ the metric is a good approximation everywhere except near the singular loci of a subset of the singular fibers (the $\mathbf{B}_{i}, \mathbf{C}_{i}$ fibers of Sec. 3.3).

\subsection{Known properties of type IIA Calabi-Yau duals $\mathcal{X}_{m, n}$}

A summary of the results obtained in Ref. [46] is as follows:

1. $\mathcal{X}_{m, n}$ is an abelian surface $\left(T^{4}\right)$ fibration over $P^{1}$, with $8+M$ singular fibers, where $M$ is number of D3-branes in $T^{6} / \mathbb{Z}_{2}$.

2. The Hodge numbers of $\mathcal{X}_{m, n}$ are $h^{11}=h^{21}=M+2$, where $m, n$, and $M$ are constrained by

$$
M+4 m n=16 .
$$

This is the D3 charge cancellation condition $N_{\mathrm{D} 3}+\int H \wedge F=N_{\mathrm{O} 3}$ on $T^{6} / \mathbb{Z}_{2}$ with $N_{\mathrm{D} 3}=M$ and $N_{\mathrm{O} 3}=16$.

3. The generic Mordell-Weil lattice of sections ( $\bmod$ torsion) of $\mathcal{X}_{m, n}$ is $D_{M}$. Here, generic means that all fibers are topologically $I_{1} \times T^{2}$. This was not mentioned explicitly in Ref. [46], but follows from the fact that $M$ D-branes plus an O-plane can coalesce to give $S O(2 N)$ enhanced gauge symmetry in $T^{6} / \mathbb{Z}_{2}$. It also follows from consideration of D3 instantons in $T^{6} / \mathbb{Z}_{2}[25]$.

4. The fundamental group and isometry group of $\mathcal{X}_{m, n}$ are

$$
\pi_{1}=\mathbb{Z}_{n} \times \mathbb{Z}_{n} \quad \text { and } \quad \mathrm{MW}_{\text {tor }}=\mathbb{Z}_{m} \times \mathbb{Z}_{m},
$$

corresponding to a disrete KK gauge symmetry and discrete winding gauge symmetry, respectively. This follows from the fact that the flux parametrizes a gauging of the low energy $\mathcal{N}=4$ supergravity theory of $T^{6} / \mathbb{Z}_{2}$ (i.e., the charges coupling scalars to vectors). For nonminimal flux $(m, n) \neq(1,1)$, the resulting superHiggs mechanism down to $\mathcal{N}=2$ only partially breaks four of the $U(1) \mathrm{s}$ of $T^{6} / \mathbb{Z}_{2}$, leaving the discrete gauge symmetry (3.5).

5. The polarization of the abelian fiber is $(\bar{m}, \bar{n})=(m, n) / \operatorname{gcd}(m, n)$. This means that the Kähler form on the fiber is proportional to a Hodge form

$$
\omega=\bar{n} d y^{1} \wedge d y^{2}+\bar{m} d y^{3} \wedge d y^{4},
$$

a positive integer form that can be used to define a projective embedding. 
6. The interchange $m \leftrightarrow n$ corresponds to T-duality of the $T^{4}$ fiber. For $m=1$, this interchange can be achieved as a quotient by the isometry group, ${ }^{20}$

$$
\mathcal{X}_{1, m}=\mathcal{X}_{m, 1} /\left(\mathbb{Z}_{m} \times \mathbb{Z}_{m}\right)
$$

7. In a convenient basis, the nonzero intersection numbers are

$$
H^{2} \cdot A=2 \bar{m} \bar{n}, \quad H \cdot \mathcal{E}_{I} \cdot \mathcal{E}_{J}=-\bar{m} \delta_{I J},
$$

where $A$ is the abelian fiber. These were deduced from the explicit harmonic forms in the approximate Calabi-Yau metric.

8. The only nonzero intersection with the second Chern class of $\mathcal{X}_{m, n}$ is

$$
H \cdot c_{2}=8+M
$$

This follows from the $F_{1}$ topological amplitude of $T^{6} / \mathbb{Z}_{2}$, which to leading order is determined by the Green-Schwarz mechanism.

Properties 1, 5, and 6 were deduced using the existence of an approximate metric on the type IIA Calabi-Yau manifold $\mathcal{X}_{m, n}$ that is exactly dual to the leading order supergravity description of the type IIB $T^{6} / \mathbb{Z}_{2}$ orientifold:

9. The approximate metric is twisted product of a Gibbons-Hawking metric and a $T^{2}$ metric,

$$
\begin{aligned}
d s_{\mathrm{CY}}^{2}= & Z\left(\frac{v_{B}}{\operatorname{Im} \tau}\left|d y^{5}+\tau d y^{6}\right|^{2}+R_{2}^{2}\left(d y^{2}\right)^{2}\right)+Z^{-1} R_{1}^{2}\left(d y^{1}+A^{1}\right)^{2} \\
& +\frac{v_{F}}{\operatorname{Im} \tau}\left|\eta^{3}+\tau \eta^{4}\right|^{2}, \quad R_{1} R_{2}=(n / m) v_{F}
\end{aligned}
$$

modulo $\mathbb{Z}_{2}\left(y^{1,2,5,6}\right)$, where $v_{F}$ and $v_{B}$ are the fiber and base Kähler moduli, respectively. The factor $Z$ satisfies a Poisson equation on $T_{\{2,3,4\}}^{3} \cdot{ }^{21}$

- For $m, n=0$, the first line (the Gibbons-Hawking part) approximates a K3 metric, and the second line is a $T^{2}$ metric.

- For $m, n \neq 0$, the two pieces are twisted:

$$
\begin{aligned}
d A^{1} & =R_{1} \star_{3} d Z-2 m\left(\eta^{3} \wedge d y^{6}-\eta^{4} \wedge d y^{5}\right), \\
d \eta^{3} & =2 n d y^{2} \wedge d y^{5}, \quad d \eta^{4}=2 n d y^{2} \wedge d y^{6} .
\end{aligned}
$$

Since the right hand side of Eq. (3.11) vanishes at fixed $y^{5}, y^{6}$, we can interpret the metric as that of a $T_{\{1,2,3,4\}}^{4}$ fibration over $T_{\{5,6\}}^{2} / \mathbb{Z}_{2} \cong \mathbb{P}^{1}$.

\footnotetext{
${ }^{20}$ In the $m=4$ case, one can also quotient in two steps: $\mathcal{X}_{2,2}=\mathcal{X}_{4,1} /\left(\mathbb{Z}_{2} \times \mathbb{Z}_{2}\right), \mathcal{X}_{1,4}=\mathcal{X}_{2,2} /\left(\mathbb{Z}_{2} \times \mathbb{Z}_{2}\right)$, where the $\mathbb{Z}_{2} \times \mathbb{Z}_{2}$ is a subgroup of $\mathbb{Z}_{4} \times \mathbb{Z}_{4}$ in the first step, and is the quotient group in the second step.

${ }^{21}$ It is the same as the warp factor of the $T^{6} / \mathbb{Z}_{2}$ orientifold, averaged over the three T-dualized directions transverse to $T_{\{2,3,4\}}^{3}$.
} 


\subsection{Monodromy matrices for the abelian surface fibrations}

In Sec. 2, we saw that the topology of an elliptic $\mathrm{K} 3$ or $\mathrm{dP}_{9}$ was determined by a branchcut-ordered set of points on $\mathbb{P}^{1}$ and the corresponding $S L(2, \mathbb{Z})$ monodromy matrices that determine how the coordinates and 1-forms on $T^{2}$ are to be identified across branch cuts. In the type IIB description, the choice is interpreted as that of a collection of $(p, q)$ 7-branes.

Likewise, the topology of $\mathcal{X}_{m, n}$ can be defined by giving an ordered set of points on the $\mathbb{P}^{1}$ base and corresponding $S L(4, \mathbb{Z})$ monodromies ${ }^{22}$ acting on $T^{4}$. And, we can again determine this information explicitly via the duality to $T^{6} / \mathbb{Z}_{2}$. In App. D, it is shown that the $M$ D3-branes (plus orientifold planes) of $T^{6} / \mathbb{Z}_{2}$ map to a collection

$$
\mathbf{A}^{M} \mathbf{B}_{1} \mathbf{C}_{1} \mathbf{B}_{2} \mathbf{C}_{2} \mathbf{B}_{3} \mathbf{C}_{3} \mathbf{B}_{4} \mathbf{C}_{4}
$$

of singular $T^{4}$ fibers of $\mathcal{X}_{m, n}$. The explicit monodromy matrices are

$$
K_{\mathbf{A}}=\left(\begin{array}{cccc}
1 & -1 & 0 & 0 \\
0 & 1 & 0 & 0 \\
0 & 0 & 1 & 0 \\
0 & 0 & 0 & 1
\end{array}\right)
$$

from the $M$ D-branes in $T^{6} / \mathbb{Z}_{2}$, and

$$
\begin{aligned}
& K_{\mathbf{C}_{1}}=\left(\begin{array}{cccc}
2 & -1 & 0 & m \\
1 & 0 & 0 & m \\
-n & n & 1 & -m n \\
0 & 0 & 0 & 1
\end{array}\right), \quad K_{\mathbf{B}_{1}}=\left(\begin{array}{cccc}
0 & -1 & 0 & -m \\
1 & 2 & 0 & m \\
-n & -n & 1 & -m n \\
0 & 0 & 0 & 1
\end{array}\right) \\
& K_{\mathbf{C}_{2}}=\left(\begin{array}{cccc}
2 & -1 & 0 & 0 \\
1 & 0 & 0 & 0 \\
0 & 0 & 1 & 0 \\
0 & 0 & 0 & 1
\end{array}\right), \quad K_{\mathbf{B}_{2}}=\left(\begin{array}{cccc}
0 & -1 & 0 & 0 \\
1 & 2 & 0 & 0 \\
0 & 0 & 1 & 0 \\
0 & 0 & 0 & 1
\end{array}\right) \\
& K_{\mathbf{C}_{3}}=\left(\begin{array}{cccc}
2 & -1 & -m & 0 \\
1 & 0 & -m & 0 \\
0 & 0 & 1 & 0 \\
-n & n & m n & 1
\end{array}\right), \quad K_{\mathbf{B}_{3}}=\left(\begin{array}{cccc}
0 & -1 & m & 0 \\
1 & 2 & -m & 0 \\
0 & 0 & 1 & 0 \\
-n & -n & m n & 1
\end{array}\right) \\
& K_{\mathbf{C}_{4}}=\left(\begin{array}{cccc}
2 & -1 & -m & m \\
1 & 0 & -m & m \\
-n & n & 1+m n & -m n \\
-n & n & m n & 1-m n
\end{array}\right), \quad K_{\mathbf{B}_{4}}=\left(\begin{array}{cccc}
0 & -1 & m & -m \\
1 & 2 & -m & m \\
-n & -n & 1+m n & -m n \\
-n & -n & m n & 1-m n
\end{array}\right) .
\end{aligned}
$$

\footnotetext{
${ }^{22}$ A collection of $S L(4, \mathbb{Z})$ monodromies defines a $T^{4}$ fibration. This $T^{4}$ fibration is an abelian surface fibration, if there also exists a monodromy invariant Hodge form $\omega$. Alternatively, if $\omega$ is specified beforehand, the monodromy matrices must lie in the subgroup $S p(\omega, \mathbb{Z}) \subset S L(4, \mathbb{Z})$ that preserves $\omega$.
} 
Note that

$$
K_{\mathbf{A}}=\left(\text { previous } K_{\mathbf{A}}\right) \oplus(\text { identity }) \text { on } T^{2} \times T^{2} \text {, }
$$

but

$$
\begin{aligned}
& K_{\mathbf{B}_{i}}=\left(\text { previous } K_{\mathbf{B}}\right) \oplus(\text { identity }) \text { on } T^{2} \times T^{2}+m, n \text { twists, } \\
& K_{\mathbf{C}_{i}}=\left(\text { previous } K_{\mathbf{C}}\right) \oplus \text { (identity) on } T^{2} \times T^{2}+m, n \text { twists, }
\end{aligned}
$$

where the $m, n$ dependent twists in $K_{\mathbf{B}_{i}}, K_{\mathbf{C}_{i}} \operatorname{mix} T_{y^{1} y^{2}}^{2}$ and $T_{y^{3} y^{4}}^{2}$ and differ for $i=1,2,3,4$. These monodromies uniquely determine the topology of $\mathcal{X}_{m, n}$, and preserve the Hodge form (3.6).

The topology of a singular A fiber consists of a smooth $T_{y^{3} y^{4}}^{2}$ times an $\mathrm{I}_{1}$ type degeneration of $T_{y^{1} y^{2}}^{2}$ in which the $y^{1}$-circle shrinks to zero size at a point on the $y^{2}$-circle. ${ }^{23}$ The singular locus is the smooth $T_{y^{3} y^{4}}^{2}$ at the location of the singularity on $T_{y^{1} y^{2}}^{2}$. The $\mathbf{B}_{i}$ and $\mathbf{C}_{i}$ monodromies are related to $K_{\mathbf{A}}$ by similarity transformations $T K_{\mathbf{A}} T^{-1}$, as described in App. D. So, the singular $\mathbf{B}_{i}$ and $\mathbf{C}_{i}$ fibers are of the same type, except that decomposition into singular and smooth $T^{2}$ differs in each case.

On the $T^{4}$ fibers, the analog of the $(p, q)$ 1-cycles of $H_{1}\left(T^{2}\right)$ in Sec. 2 is the group of $(p, q, r, s)$ 1-cycles of $H_{1}\left(T^{4}\right)$. For each $\mathbf{A}, \mathbf{B}_{i}$ and $\mathbf{C}_{i}$ singular $T^{4}$ fiber, Table 3 lists the vanishing 1-cycle, the location of the singular locus, and a pair of nonvanishing 1-cycles spanning the singular locus (which is a smooth $T^{2}$ ). The vanishing 1-cycle and spanning cycles of the singular locus are invariant under the monodromy action associated to the singular fiber. In Table 3, the spanning cycles of the smooth $T^{2}$ are defined only modulo the vanishing cycle, and represent one choice out of many possible bases. Note that in contrast to the $T^{2}$ fibered case, specifying the vanishing cycle does not uniquely determine the $S L(4, \mathbb{Z})$ monodromy. The singular locus must also be specified.

\subsubsection{Fundamental group}

The vanishing cycles in Table 3 are trivial in $H_{1}\left(\mathcal{X}_{m, n}, \mathbb{Z}\right)$. By taking linear combinations, we deduce that the cycles $(1,0,0,0),(0,1,0,0),(0,0, n, 0)$ and $(0,0,0, n) \in H_{1}\left(\mathcal{X}_{m, n}, \mathbb{Z}\right)$ are trivial, and generate all trivial cycles. Thus, $(0,0,1,0)$ and $(0,0,0,1)$ generate $\mathbb{Z}_{n}$ torsion cycles, and

$$
H_{1}\left(\mathcal{X}_{m, n}, \mathbb{Z}\right)=\mathbb{Z}_{n} \times \mathbb{Z}_{n} .
$$

Provided that $\pi_{1}\left(\mathcal{X}_{m, n}\right)$ is abelian, ${ }^{24}$ this implies that $\pi_{1}=\mathbb{Z}_{n} \times \mathbb{Z}_{n}$, in agreement with the result (3.5) obtained from the low energy effective field theory of $T^{6} / \mathbb{Z}_{2}$. Indeed, the inclusion $A \hookrightarrow \mathcal{X}_{m, n}$ induces a surjective map from $\pi_{1}(A)$ to $\pi_{1}\left(\mathcal{X}_{m, n}\right)$; that is, every nontrivial element

\footnotetext{
${ }^{23}$ While the singular fibers have $\mathrm{I}_{1} \times T^{2}$ topology, the complex structure need not respect this factorization. A description of the complex structure of the singular fibers is given in Sec. 4.1.

${ }^{24}$ In general, $H_{1}(\mathcal{X}, \mathbb{Z})$ is the abelianization of $\pi_{1}(\mathcal{X})$, i.e., $\pi_{1}$ modulo its commutator subgroup.
} 
Singular locus ( $\operatorname{smooth} T^{2}$ )

Singular fiber Vanishing cycle Location of singular locus

spanning cycles
A $\quad(1,0,0,0)$
$y^{2}=c$
$(0,0,1,0), \quad(0,0,0,1)$
$\mathbf{B}_{1} \quad(1,-1, n, 0)$
$y^{1}+y^{2}+m y^{4}=c$
$(0,0,1,0), \quad(-m, 0,0,1)$
$\mathrm{C}_{1} \quad(1,1,-n, 0)$
$y^{1}-y^{2}+m y^{4}=c$
$(0,0,1,0), \quad(-m, 0,0,1)$
$\mathrm{B}_{2}$
$(1,-1,0,0)$
$y^{1}+y^{2}=c$
$(0,0,1,0), \quad(0,0,0,1)$
$\mathbf{C}_{2} \quad(1,1,0,0) \quad y^{1}-y^{2}=c$
$(0,0,1,0), \quad(0,0,0,1)$
$\mathbf{B}_{3} \quad(1,-1,0, n)$
$y^{1}+y^{2}-m y^{3}=c$
$(m, 0,1,0), \quad(0,0,0,1)$
$\mathbf{C}_{3} \quad(1,1,0,-n) \quad y^{1}-y^{2}-m y^{3}=c$
$(m, 0,1,0), \quad(0,0,0,1)$
$\mathbf{B}_{4} \quad(1,-1, n, n)$
$y^{1}+y^{2}-m y^{3}+m y^{4}=c$
$(m, 0,1,0), \quad(-m, 0,0,1)$
$\mathbf{C}_{4} \quad(1,1,-n,-n)$
$y^{1}-y^{2}-m y^{3}+m y^{4}=c$
$(m, 0,1,0), \quad(-m, 0,0,1)$

Table 3: Structure of the singular $\mathbf{A}, \mathbf{B}_{i}$, and $\mathbf{C}_{i}$ fibers of the abelian surface fibration $\mathcal{X}_{m, n}$.

of $\pi_{1}\left(\mathcal{X}_{m, n}\right)$ can be deformed to lie entirely in the abelian fiber over a single point of the base. So, $\pi_{1}\left(\mathcal{X}_{m, n}\right)$ is abelian.

\subsubsection{Calabi-Yau dual interpretation of $T^{6} / \mathbb{Z}_{2} \mathbf{R R}$ tadpole}

Since the base of $\mathcal{X}_{m, n}$ is $\mathbb{P}^{1}$, a loop that encloses all singular fibers is contractible (to the point at infinity). Therefore, as in Sec. 2, the total monodromy must be unity. This gives

$$
\begin{aligned}
1 & =K_{\text {total }} \\
& =K_{\mathbf{C}_{4}} K_{\mathbf{B}_{4}} K_{\mathbf{C}_{3}} K_{\mathbf{B}_{3}} K_{\mathbf{C}_{2}} K_{\mathbf{B}_{2}} K_{\mathbf{C}_{1}} K_{\mathbf{B}_{1}} K_{\mathbf{A}}{ }^{M} \\
& =\left(\begin{array}{cccc}
1 & 0 & 0 & 0 \\
& 1 & -Q & 0 \\
& & 1 & 0 \\
& & & 1
\end{array}\right), \quad \text { where } Q=M-16+4 m n,
\end{aligned}
$$

so that $Q=0$. The topological constraint $K_{\text {total }}=1$ reproduces the $T^{6} / \mathbb{Z}_{2}$ D3 charge cancellation condition (3.4).

\subsection{Mordell-Weil lattice from junction lattice}

For an abelian surface fibration, we define the junction lattice $J$ and related lattices $J_{\text {loop }}$, $J_{\text {eff }}, J_{\text {Kodaira }}$, and $J_{0}$ exactly as in Sec. 2 , except that we now consider graphs on $\mathbb{P}^{1}$ in which each oriented string: (i) is labeled by four charges $(p, q, r, s)$, instead of two, and (ii) can terminate either at a vertex or at the location of a singular $\mathbf{X}_{[p, q, r, s]}$ fiber in which a $(p, q, r, s)$ 1-cycle shrinks. 
To define the equivalence classes of junctions, we let $\mathbf{s}_{i}$ denote an outward oriented $\left(p_{i}, q_{i}, r_{i}, s_{i}\right)$ string emanating from $\mathbf{X}_{\left[p_{i}, q_{i}, r_{i}, s_{i}\right]}$. Then, to each string junction in standard (tree) presentation, we associate a charge vector

$$
Q=\sum_{i} Q_{i} \mathbf{s}_{i},
$$

where $Q_{i} \in \mathbb{Z}$ is the net number of strings leaving (minus entering) the location of the $i$ th singular fiber, $\mathbf{X}_{\left[p_{i}, q_{i}, r_{i}, s_{i}\right]}$. In this case, we treat the strings as strictly mathematical objects, in contrast to the physical IIB strings of Sec. $2 .{ }^{25}$

To apply the inner product of Sec. 2, we use the fact that an abelian surface fibration admits a projective embedding, with corresponding Hodge form $\omega$. The Poincaré dual divisor class can be represented as the sum of positive integer multiples of two $T^{2} \mathrm{~S} .{ }^{26}$ So, we can first intersect with Hodge class and then use the inner product (2.9) on each $T^{2}$.

For the abelian surfaces fibration $\mathcal{X}_{m, n}$, the Hodge form $\omega$ was given in Eq. (3.6). As a check, it can be readily verified that $\omega$ is invariant under the monodromies (3.13) and (3.14). In this case, the Poincaré dual divisor class can be represented by $\bar{m} T_{y^{1} y^{2}}^{2}+\bar{n} T_{y^{3} y^{4}}^{2}$, and the charges $\left(p_{i}, q_{i}, r_{i}, s_{i}\right)$ have a corresponding decomposition $\left(p_{i}, q_{i}\right) \oplus\left(r_{i}, s_{i}\right)$. We define the inner product to be

$$
\begin{aligned}
& \left(s_{i}, s_{i}\right)=-1, \\
& \left(s_{i}, s_{j}\right)=\left(s_{j}, s_{i}\right)=\frac{\bar{m}}{2}\left(p_{i} q_{j}-p_{j} q_{i}\right)+\frac{\bar{n}}{2}\left(r_{i} s_{j}-r_{j} s_{i}\right), \quad i<j .
\end{aligned}
$$

For the vanishing cycles $\left(p_{i}, q_{i}, r_{i}, s_{i}\right)$ listed in Table 3 , the nonvanishing contribution to this inner product comes entirely ${ }^{27}$ from the first $T^{2}$ :

$$
\begin{aligned}
& \left(s_{i}, s_{i}\right)=-1, \\
& \left(s_{i}, s_{j}\right)=\left(s_{j}, s_{i}\right)=\frac{\bar{m}}{2}\left(p_{i} q_{j}-p_{j} q_{i}\right), \quad i<j .
\end{aligned}
$$

However, the $\left(r_{i}, s_{i}\right)$ data still show up in the junction lattice via the $(p, q, r, s)$ charge conservation conditions at vertices and the terminination conditions at the locations of singular fibers.

\footnotetext{
${ }^{25}$ What carries over directly is the interpretation of a $(p, q, r, s)$ string as the projection to $\mathbb{P}^{1}$ of a 2-cycle in the abelian fibration, whose inverse image at each point is a $(p, q, r, s) 1$-cycle in the abelian fiber. So, from the M-theory perspective, these string graphs have an analogous physical interpretation to the $(p, q)$ string of type IIB: they are M2-branes wrapped on $(p, q, r, s)$ cycles of the fiber of $\mathcal{X}_{m, n}$.

${ }^{26} \mathrm{In}$ fact, one of the two integers must be unity (cf. App. B.) This is indeed the case for the possible values of $(\bar{m}, \bar{n})$ of $\mathcal{X}_{m, n}$.

${ }^{27}$ This statement, while true, is not obvious. For example, if the base were noncompact, then the inner product between a $(1,-1, n, 0)$ string leaving a $\mathbf{B}_{1}$ point and a string leaving a $\mathbf{B}_{3}, \mathbf{C}_{3}, \mathbf{B}_{4}$ or $\mathbf{C}_{4}$ point would have a contribution from the second term in Eq. (3.20). However, it can be shown that the inner product between proper string junctions with no asymptotic $(p, q)$ charge would not. In the compact case, the only junctions are the proper junctions.
} 
For the collection (3.12), with no coalesced 7-branes, the resulting junction lattice is

$$
J=D_{M}^{\bar{m}-} \oplus J_{\text {null }}, \quad \text { where } \quad J_{\text {null }}=\boldsymbol{\delta}_{1} \mathbb{Z} \oplus \boldsymbol{\delta}_{2} \mathbb{Z} \oplus \boldsymbol{\delta}_{3} \mathbb{Z} \oplus \boldsymbol{\delta}_{4} \mathbb{Z} .
$$

In the first term, the superscript indicates that the (positive definite) inner product is $-\bar{m}$ times the inner product of the root lattice of $D_{M}$. The $D_{M}$ is generated by the proper junction lattice of $\mathbf{A}^{M} \mathbf{B}_{i} \mathbf{C}_{i}$, for any one choice of $i$, just as in the elliptically fibered case (cf. Refs. $[15,16]$ ). However, unlike the case of an elliptic $\mathrm{K} 3$ or $\mathrm{dP}_{9}$ in Sec. 2, we do not obtain an $E_{M+1}$ lattice from the proper junctions of $\mathbf{A}^{M} \mathbf{B}_{i} \mathbf{C}_{i} \mathbf{C}_{j}$. The fact that $\mathbf{C}_{i}$ and $\mathbf{C}_{j}$ have different $(p, q, r, s)$ for $i \neq j$ means that the analog of the $E_{M+1}$ enhancing root does not exist for this abelian fibration.

The lattice $J_{\text {null }}$ in Eq. (3.22) is the null sublattice of the junction lattice. A basis of generators is

$$
\begin{aligned}
& \boldsymbol{\delta}_{1}=\left(0^{M} ;-1,-1 ; 1,1 ;-1,-1 ; 1,1\right), \\
& \boldsymbol{\delta}_{2}=\left((-1)^{M} ; M-1, M-3 ; 5-M, 7-M ; M / 2-1, M / 2-3 ;-3,-1\right), \\
& \boldsymbol{\delta}_{3}=\left(0^{M} ; 0,0 ; 0,0 ; 1,1,-1,-1\right), \\
& \boldsymbol{\delta}_{4}=\left(0^{M} ;-1,-1 ; 2,2 ;-2,-2 ; 1,1\right) .
\end{aligned}
$$

In App. E, we compute the lattice $J_{\text {loop }}$ generated by null loop junctions. We find

$$
J_{\text {loop }}=\boldsymbol{\delta}_{1} \mathbb{Z} \oplus \boldsymbol{\delta}_{2} \mathbb{Z} \oplus m \boldsymbol{\delta}_{3} \mathbb{Z} \oplus m \boldsymbol{\delta}_{4} \mathbb{Z},
$$

corresponding to loops with $(p, q, r, s)=(1,0,0,0),(0,1,0,0),(0,0,1,0)$, and $(0,0,0,1)$, respectively. Therefore, the effective junction lattice is

$$
J_{\text {eff }}=J / J_{\text {loop }}=D_{M}^{\bar{m}-} \oplus \boldsymbol{\delta}_{3} \mathbb{Z}_{m} \oplus \boldsymbol{\delta}_{4} \mathbb{Z}_{m} .
$$

Finally, $J_{\text {Kodaira }}$ is trivial, since there are no coalesced branes. Therefore, $J_{0}=J_{\text {eff }}$, and there is no distinction between integral and weakly integral junctions. We have

$$
\begin{aligned}
\mathrm{MW}=\mathrm{MW}_{0} & =J_{0}=D_{M}^{\bar{m}-} \oplus \mathbb{Z}_{\bar{m}} \oplus \mathbb{Z}_{\bar{m}}, \\
\mathrm{MW}_{\text {tor }} & =J_{\text {null }} / J_{\text {loop }}=\mathbb{Z}_{\bar{m}} \oplus \mathbb{Z}_{\bar{m}},
\end{aligned}
$$

exactly as predicted via effective field theory considerations in Ref. [46].

Note that the narrow Mordell-Weil lattice $\mathrm{MW}_{0}$ has torsion. This distinguishes abelian surface fibrations from elliptic fibrations over $\mathbb{P}^{1}$, where such torsion cannot occur. (In terms of the junction lattices, $J_{\text {null }}=J_{\text {loop }}$ for elliptic surfaces.)

In fact, it is easy to identify the torsion sections explicitly. The sections

$$
\begin{array}{lll}
\boldsymbol{\delta}_{3} \cong\left\{\left(y^{1}, y^{2}, y^{3}, y^{4}\right)=\left(0,0, \frac{1}{m}, 0\right)\right. & \text { in } \left.\mathcal{X}_{m, n}\right\} & \left(\bmod \text { loops } \boldsymbol{\delta}_{1}, \boldsymbol{\delta}_{2}, m \boldsymbol{\delta}_{3}, m \boldsymbol{\delta}_{4}\right), \\
\boldsymbol{\delta}_{4} \cong\left\{\left(y^{1}, y^{2}, y^{3}, y^{4}\right)=\left(0,0,0, \frac{1}{m}\right)\right. & \text { in } \left.\mathcal{X}_{m, n}\right\} & \left(\bmod \text { loops } \boldsymbol{\delta}_{1}, \boldsymbol{\delta}_{2}, m \boldsymbol{\delta}_{3}, m \boldsymbol{\delta}_{4}\right),
\end{array}
$$

are invariant under the monodromy actions (3.13) and (3.14) up to the identifications $y^{i} \cong$ $y^{i}+1$. 


\subsection{Examples with coalesced fibers}

We now consider three examples of collections with coalesced fibers. In the first example, we assume that $M=16-4 m n \geq 4$ and obtain an enhancement of $\mathrm{MW}_{\text {tor }}\left(\mathcal{X}_{m, n}\right)$ from $\mathbb{Z}_{m} \oplus \mathbb{Z}_{m}$ to $\mathbb{Z}_{2 m} \oplus \mathbb{Z}_{m}$. In the next two examples, we restrict to the principally polarized case $(m, n)=(1,1)$. We identify collections leading to $\mathbb{Z}_{2}{ }^{\oplus 4}$ and $\mathbb{Z}_{4} \oplus \mathbb{Z}_{2}{ }^{\oplus 2}$ torsion, respectively. In Sec. 3.6, we describe how new abelian surface fibered Calabi-Yau manifolds can be obtained by quotienting by these isometry groups.

\subsection{1 $\mathbb{Z}_{2 m} \oplus \mathbb{Z}_{m}$ torsion, $m=1,2,3$}

From the braiding operations discussed in App. A, we have

$$
\begin{aligned}
\mathbf{A}^{M} \mathbf{B}_{1} \mathbf{C}_{1} \mathbf{B}_{2} \mathbf{C}_{2} \mathbf{B}_{3} \mathbf{C}_{3} \mathbf{B}_{4} \mathbf{C}_{4} & \cong \mathbf{A}^{M-4} \mathbf{B}_{1} \mathbf{C}_{1} \mathbf{B}_{2} \mathbf{C}_{2} \mathbf{B}_{3} \mathbf{C}_{3} \mathbf{A}^{2} \mathbf{B}_{4} \mathbf{C}_{4} \mathbf{A}^{2} \\
& \cong \mathbf{A}^{M-4} \mathbf{B}_{1} \mathbf{C}_{1} \mathbf{B}_{2} \mathbf{C}_{2} \mathbf{B}_{3} \mathbf{A}^{2} \mathbf{B}_{3} \mathbf{B}_{4} \mathbf{A}^{2} \mathbf{B}_{4} \\
& \cong \mathbf{A}^{M-4} \mathbf{B}_{1} \mathbf{C}_{1} \mathbf{B}_{2} \mathbf{C}_{2} \mathbf{B}_{3}{ }^{2} \mathbf{D}_{3}{ }^{2} \mathbf{B}_{4}{ }^{2} \mathbf{D}_{4}{ }^{2}
\end{aligned}
$$

where the $\mathbf{D}_{i}$ are defined in App. A and have vanishing cycles of the form $(0,1, *, *)$. In the basis corresponding to the last collection of Eq. (3.31), the generators (3.23) of $J_{\text {null }}$ become

$$
\begin{aligned}
& \boldsymbol{\delta}_{1}=\left(0^{M-4} ;-1,-1 ; 1,1 ;-1,-1,-1,-1 ; 1,1,1,1\right), \\
& \boldsymbol{\delta}_{2}=\left(1^{M-4} ; M-5, M-7 ; 9-M, 11-M ;(5-M / 2)^{2},(M / 2-6)^{2} ;(-1)^{2}, 0^{2}\right), \\
& \boldsymbol{\delta}_{3}=\left(0^{M-4} ; 0,0 ; 0,0 ; m, m, m, m ;-m,-m,-m,-m\right), \\
& \boldsymbol{\delta}_{4}=\left(0^{M-4} ;-m,-m ; 2 m, 2 m ;-2 m,-2 m,-2 m,-2 m ; m, m, m, m\right) .
\end{aligned}
$$

The loop junction lattice $J_{\text {loop }}$ is given by Eq. (3.27).

Now, suppose that we coalesce 7-branes pairwise to obtain the collection

$$
\mathbf{A}^{M-4} \mathbf{B}_{1} \mathbf{C}_{1} \mathbf{B}_{2} \mathbf{C}_{2}\left(\mathbf{B}_{3}{ }^{2}\right)\left(\mathbf{D}_{3}{ }^{2}\right)\left(\mathbf{B}_{4}{ }^{2}\right)\left(\mathbf{D}_{4}{ }^{2}\right) \text {. }
$$

Then,

$$
J_{\text {Kodaira }}=A_{1}^{-\oplus 4}
$$

corresponding to 4 singular fibers each containing an elliptic curves of $A_{1}$ singularities. In the coalesced collection $(3.33), \boldsymbol{\delta}_{3} / 2$ becomes a weakly integral null junction, so that

$$
J_{\text {null }}^{\text {weak }}=\boldsymbol{\delta}_{1} \mathbb{Z} \oplus \boldsymbol{\delta}_{2} \mathbb{Z} \oplus\left(\boldsymbol{\delta}_{3} / 2\right) \mathbb{Z} \oplus \boldsymbol{\delta}_{4} \mathbb{Z} .
$$

and the Mordell-Weil torsion is

$$
\mathrm{MW}_{\text {tor }}=J_{\text {null }}^{\text {weak }} / J_{\text {loop }}=\boldsymbol{\delta}_{3} \mathbb{Z}_{2 m} \oplus \boldsymbol{\delta}_{4} \mathbb{Z}_{m} .
$$

As in Sec. 3.4, the torsion sections can be described explicitly in terms of the coordinates $y^{i}$ on the abelian fiber. They are generated by the sections

$$
\left(y^{1}, y^{2}, y^{3}, y^{4}\right)=\left(0,0, \frac{1}{2 m}, 0\right) \text { and }\left(0,0,0, \frac{1}{m}\right) \text { in } \mathcal{X}_{m, n},
$$

which are easily seen to be monodromy invariant, up to the identifications $y^{i} \cong y^{i}+1$. 


\subsection{2 $\mathbb{Z}_{2}^{\oplus 2} \oplus \mathbb{Z}_{2 m}^{\oplus 2}$ torsion, $m=1,2$}

In the case that $M=16-4 m n \geq 8$, the isometry group of torsion sections can be further enhanced to $\mathbb{Z}_{2}{ }^{\oplus 2} \oplus \mathbb{Z}_{2 m}{ }^{\oplus 2}$ by coalescing additional fibers. Consider a coalesced collection analogous to that of Sec. 2.5.3. From the braiding operations discussed in App. A, we have

$$
\begin{aligned}
\mathbf{A}^{M} \mathbf{B}_{1} \mathbf{C}_{1} \mathbf{B}_{2} \mathbf{C}_{2} \mathbf{B}_{3} \mathbf{C}_{3} \mathbf{B}_{4} \mathbf{C}_{4} & \cong \mathbf{A}^{M-8} \mathbf{B}_{1} \mathbf{C}_{1} \mathbf{A}^{2} \mathbf{B}_{2} \mathbf{C}_{2} \mathbf{A}^{2} \mathbf{B}_{3} \mathbf{C}_{3} \mathbf{A}^{2} \mathbf{B}_{4} \mathbf{C}_{4} \mathbf{A}^{2} \\
& \cong \mathbf{A}^{M-8} \mathbf{B}_{1} \mathbf{A}^{2} \mathbf{B}_{1} \mathbf{B}_{2} \mathbf{A}^{2} \mathbf{B}_{2} \mathbf{B}_{3} \mathbf{A}^{2} \mathbf{B}_{3} \mathbf{B}_{4} \mathbf{A}^{2} \mathbf{B}_{4} \\
& \cong \mathbf{A}^{M-8} \mathbf{B}_{1}^{2} \mathbf{D}_{1}^{2} \mathbf{B}_{2}^{2} \mathbf{D}_{2}^{2} \mathbf{B}_{3}^{2} \mathbf{D}_{3}^{2} \mathbf{B}_{4}^{2} \mathbf{D}_{4}^{2}
\end{aligned}
$$

In the basis corresponding to the last collection of Eq. (3.38), the generators (3.23) of $J_{\text {null }}$ become

$\boldsymbol{\delta}_{1}=\left(0^{M-8} ;(-1)^{4} ; 1^{4} ;(-1)^{4} ; 1^{4}\right)$,

$\boldsymbol{\delta}_{2}=\left((-1)^{M-8} ;(M-9)^{2},(M-10)^{2} ;(11-M)^{2},(12-M)^{2} ;(M / 2-5)^{2},(M / 2-6)^{2} ;(-1)^{2}, 0^{2}\right)$,

$\boldsymbol{\delta}_{3}=\left(0^{M-8} ; 0^{4} ; 0^{4} ; m^{4} ;(-m)^{4}\right)$,

$\boldsymbol{\delta}_{4}=\left(0^{M-8} ;(-m)^{4} ;(2 m)^{4} ;(-2 m)^{4} ; m^{4}\right)$.

The loop junction lattice $J_{\text {loop }}$ is given by Eq. (3.27).

Now, suppose that we coalesce fibers pairwise to obtain the collection

$$
\left(\mathbf{A}^{2}\right)\left(\mathbf{A}^{2}\right)\left(\mathbf{B}_{1}^{2}\right)\left(\mathbf{D}_{1}^{2}\right)\left(\mathbf{B}_{2}^{2}\right)\left(\mathbf{D}_{2}^{2}\right)\left(\mathbf{B}_{3}^{2}\right)\left(\mathbf{D}_{3}^{2}\right)\left(\mathbf{B}_{4}^{2}\right)\left(\mathbf{D}_{4}^{2}\right)
$$

Then,

$$
J_{\text {Kodaira }}=A_{1}^{-\oplus 10}
$$

corresponding to 10 singular fibers, equal to the compactified Jacobian of a genus-2 curve with an $\mathrm{I}_{2}$ type degeneration, or equivalently, each with an elliptic curve of $A_{1}$ singularities. Each $\boldsymbol{\delta}_{i} / 2$ becomes a weakly integral null junction, so that

$$
J_{\text {null }}^{\text {weak }}=\left(\boldsymbol{\delta}_{1} / 2\right) \mathbb{Z} \oplus\left(\boldsymbol{\delta}_{2} / 2\right) \mathbb{Z} \oplus\left(\boldsymbol{\delta}_{3} / 2\right) \mathbb{Z} \oplus\left(\boldsymbol{\delta}_{4} / 2\right) \mathbb{Z} \text {. }
$$

and the Mordell-Weil torsion is

$$
\mathrm{MW}_{\text {tor }}=J_{\text {null }}^{\text {weak }} / J_{\text {loop }}=\left(\boldsymbol{\delta}_{1} / 2\right) \mathbb{Z}_{2} \oplus\left(\boldsymbol{\delta}_{1} / 2\right) \mathbb{Z}_{2} \oplus\left(\boldsymbol{\delta}_{1} / 2\right) \mathbb{Z}_{2 m} \oplus\left(\boldsymbol{\delta}_{1} / 2\right) \mathbb{Z}_{2 m}
$$

The torsion sections can again be described explicitly in terms of the coordinates $y^{i}$ on the abelian fiber. They are generated by the sections

$$
\left(y^{1}, y^{2}, y^{3}, y^{4}\right)=\left(\frac{1}{2}, 0,0,0\right),\left(0, \frac{1}{2}, 0,0\right),\left(0,0, \frac{1}{2 m}, 0\right), \text { and }\left(0,0,0, \frac{1}{2 m}\right) \text { in } \mathcal{X}_{1,1},
$$

which are easily seen to be monodromy invariant, up to the identifications $y^{i} \cong y^{i}+1$. 
Finally, torsion subgroups can be obtained by partially uncoalescing the collection. For example, if we uncoalesce

the $\left(\mathbf{A}^{2}\right) \mathrm{s} \Rightarrow$ we obtain $\mathrm{MW}_{\text {tor }}=\mathbb{Z}_{2} \oplus \mathbb{Z}_{2 m}{ }^{\oplus 2}$, generated by $\boldsymbol{\delta}_{1} / 2, \boldsymbol{\delta}_{3} / 2, \boldsymbol{\delta}_{4} / 2$,

and $\left(\mathbf{B}_{2}^{2}\right),\left(\mathbf{D}_{2}^{2}\right) \Rightarrow$ we obtain $\mathrm{MW}_{\text {tor }}=\mathbb{Z}_{2 m}{ }^{\oplus 2}$, generated by $\boldsymbol{\delta}_{3} / 2, \boldsymbol{\delta}_{4} / 2$,

and $\left(\mathbf{B}_{3}^{2}\right),\left(\mathbf{D}_{3}^{2}\right) \quad \Rightarrow \quad$ we obtain $\mathrm{MW}_{\text {tor }}=\mathbb{Z}_{m} \oplus \mathbb{Z}_{2 m}$, generated by $\boldsymbol{\delta}_{3}, \boldsymbol{\delta}_{4} / 2$.

\subsection{3 $\quad \mathbb{Z}_{4} \oplus \mathbb{Z}_{2}^{\oplus 2}$ torsion and $\mathbb{Z}_{2}^{\oplus 3}$ torsion}

Now focus on the principally polarized case $(m, n)=(1,1)$. From the braiding operations discussed in App. A, we have

$$
\begin{aligned}
\mathbf{A}^{12} \mathbf{B}_{1} \mathbf{C}_{1} \mathbf{B}_{2} \mathbf{C}_{2} \mathbf{B}_{3} \mathbf{C}_{3} \mathbf{B}_{4} \mathbf{C}_{4} & \cong \mathbf{A}^{3} \mathbf{B}_{1} \mathbf{C}_{1} \mathbf{A}^{3} \mathbf{B}_{2} \mathbf{C}_{2} \mathbf{A}^{3} \mathbf{B}_{3} \mathbf{C}_{3} \mathbf{A}^{3} \mathbf{B}_{4} \mathbf{C}_{4} \\
& \cong \mathbf{D}_{1}^{4} \mathbf{E}_{1} \mathbf{D}_{2}{ }^{4} \mathbf{E}_{2} \mathbf{D}_{3}{ }^{4} \mathbf{E}_{3} \mathbf{D}_{4}{ }^{4} \mathbf{E}_{4},
\end{aligned}
$$

where the $\mathbf{E}_{i}$ are defined in App. A and have vanishing cycles of the form $(1,2, *, *)$. In the basis corresponding to the last collection of Eq. (3.46), the generators (3.23) of $J_{\text {null }}$ become

$$
\begin{aligned}
& \boldsymbol{\delta}_{1}=\left(1^{4},-2 ;(-1)^{4}, 2 ; 1^{4},-2 ;(-1)^{4}, 2\right), \\
& \boldsymbol{\delta}_{2}=\left(0^{4},-1 ;(-1)^{4}, 3 ; 0^{4},-1 ; 1^{4},-1\right), \\
& \boldsymbol{\delta}_{3}=\left(0^{10} ;(-1)^{4}, 2 ; 1^{4},-2\right), \\
& \boldsymbol{\delta}_{4}=\left(1^{4},-2 ; 2^{4}, 4 ; 2^{4},-4 ;(-1)^{4}, 2\right) .
\end{aligned}
$$

The loop junction lattice $J_{\text {loop }}$ is Eq. (3.27) with $m=1$.

Now, suppose that we coalesce quadruples of fibers to obtain the collection

$$
\left(\mathbf{D}_{1}^{4}\right) \mathbf{E}_{1}\left(\mathbf{D}_{2}^{4}\right) \mathbf{E}_{2}\left(\mathbf{D}_{3}^{4}\right) \mathbf{E}_{3}\left(\mathbf{D}_{4}^{4}\right) \mathbf{E}_{4}
$$

Then,

$$
J_{\text {Kodaira }}=A_{3}^{-\oplus 4},
$$

corresponding to 4 singular fibers equal to the compactified Jacobian of a genus- 2 curve with an $\mathrm{I}_{4}$ type degeneration, or equivalentally, 4 elliptic curves of $A_{3}$ singularities. For weakly integral null junctions, the charges of the $\mathbf{D}_{i}$ must be 1/4-integral and those of the $\mathbf{E}_{i}$ must be integral. This gives weakly integral null junctions $\boldsymbol{\delta}_{1} / 4-\boldsymbol{\delta}_{2} / 2, \boldsymbol{\delta}_{3} / 2$, and $\boldsymbol{\delta}_{4} / 2$, with

$$
\mathrm{MW}_{\text {tor }}=J_{\text {null }}^{\text {weak }} / J_{\text {loop }}=\left(\boldsymbol{\delta}_{1} / 4-\boldsymbol{\delta}_{2} / 2\right) \mathbb{Z}_{4} \oplus\left(\boldsymbol{\delta}_{3} / 2\right) \mathbb{Z}_{2} \oplus\left(\boldsymbol{\delta}_{4} / 2\right) \mathbb{Z}_{2} .
$$

In terms of the coordinates $y^{i}$ on the abelian fiber, the explicit torsion sections are

$$
\left(y^{1}, y^{2}, y^{3}, y^{4}\right)=(1 / 4,1 / 2,0,0),(0,0,1 / 2,0), \text { and }(0,0,0,1 / 2)
$$


respectively.

If instead of coalescing quadruples, we coalesce pairs of $\mathbf{D}_{i}$ fibers,

$$
\left(\mathbf{D}_{1}^{2}\right)\left(\mathbf{D}_{1}^{2}\right) \mathbf{E}_{1}\left(\mathbf{D}_{2}^{2}\right)\left(\mathbf{D}_{2}^{2}\right) \mathbf{E}_{2}\left(\mathbf{D}_{3}^{2}\right)\left(\mathbf{D}_{3}^{2}\right) \mathbf{E}_{3}\left(\mathbf{D}_{4}^{2}\right)\left(\mathbf{D}_{4}^{2}\right) \mathbf{E}_{4}
$$

then

$$
J_{\text {Kodaira }}=A_{1}^{-\oplus 8},
$$

corresponding to 8 singular fibers equal to the compactified Jacobian of a genus- 2 curve with an $\mathrm{I}_{2}$ type degeneration, or equivalentally, 4 elliptic curves of $A_{1}$ singularities. The Mordell-Weil torsion is reduced from $\mathbb{Z}_{4} \oplus \mathbb{Z}_{2}{ }^{\oplus 2}$ to $\mathbb{Z}_{2}{ }^{\oplus 3}$ :

$$
\mathrm{MW}_{\text {tor }}=J_{\text {null }}^{\text {weak }} / J_{\text {loop }}=\left(\boldsymbol{\delta}_{1} / 2\right) \mathbb{Z}_{2} \oplus\left(\boldsymbol{\delta}_{3} / 2\right) \mathbb{Z}_{2} \oplus\left(\boldsymbol{\delta}_{4} / 2\right) \mathbb{Z}_{2} \text {. }
$$

In Sec. 4, we will reproduce this last result from the relative Jacobian construction of $\mathcal{X}_{1,1}$.

\subsection{Connections to other Calabi-Yau manifolds with nontrivial $\pi_{1}$}

Let us focus on the example in Sec. 3.5.1 in the principally polarized case $(m, n)=(1,1)$. The collection is

$$
\mathbf{A}^{8} \mathbf{B}_{1} \mathbf{C}_{1} \mathbf{B}_{2} \mathbf{C}_{2}\left(\mathbf{B}_{3}^{2}\right)\left(\mathbf{D}_{3}^{2}\right)\left(\mathbf{B}_{4}^{2}\right)\left(\mathbf{D}_{4}^{2}\right)
$$

with

$$
J_{\text {Kodaira }}=A_{1}^{-\oplus 4} \quad \text { and } \quad \mathrm{MW}_{\text {tor }}=J_{\text {null }}^{\text {weak }} / J_{\text {loop }}=\mathbb{Z}_{2} .
$$

In this case, the Calabi-Yau manifold $\mathcal{X}_{1,1}$ has a $\mathbb{Z}_{2}$ isometry. In terms of the coordinates $y^{1}, y^{2}, y^{3}, y^{4}$ on the abelian surface fiber, the isometry is $y^{3} \mapsto y^{3}+1 / 2$.

We now quotient by this isometry and ask what resulting topology is obtained. Since we would like preserve the condition that the fiber coordinates are periodic modulo 1 , we will rescale the $y^{3}$ coordinate so that $y_{\text {new }}^{3}=2 y_{\text {old }}^{3}$. This is implemented by conjugating all monodromy matrices:

$$
K_{\text {old }} \mapsto K_{\text {new }}=T K_{\text {old }} T^{-1}, \quad \text { where } \quad T=\operatorname{diag}(1,1,2,1) .
$$

The conjugation leaves $K_{\mathbf{A}}, K_{\mathbf{B}_{2}}$ and $K_{\mathbf{C}_{2}}$ unchanged, and maps the monodromy matrices 
$K_{\mathbf{C}_{1}}, K_{\mathbf{B}_{1}}, K_{\left(\mathbf{D}_{3}\right)^{2}}, K_{\left(\mathbf{B}_{3}\right)^{2}}, K_{\left(\mathbf{D}_{4}\right)^{2}}$, and $K_{\left(\mathbf{B}_{4}\right)^{2}}$ to

$$
\begin{aligned}
& K_{\widetilde{\mathbf{C}}_{1}}=\left(\begin{array}{cccc}
2 & -1 & 0 & 1 \\
1 & 0 & 0 & 1 \\
-2 & 2 & 1 & -2 \\
0 & 0 & 0 & 1
\end{array}\right), \quad K_{\widetilde{\mathbf{B}}_{1}}=\left(\begin{array}{cccc}
0 & -1 & 0 & -1 \\
1 & 2 & 0 & 1 \\
-2 & -2 & 1 & -2 \\
0 & 0 & 0 & 1
\end{array}\right), \\
& K_{\widetilde{\mathbf{D}}_{3}}=\left(\begin{array}{cccc}
1 & 0 & 0 & 0 \\
2 & 1 & -1 & 0 \\
0 & 0 & 1 & 0 \\
-2 & 0 & 1 & 1
\end{array}\right), \quad K_{\widetilde{\mathbf{B}}_{3}}=\left(\begin{array}{cccc}
-1 & -2 & 1 & 0 \\
2 & 3 & -1 & 0 \\
0 & 0 & 1 & 0 \\
-2 & -2 & 1 & 1
\end{array}\right) \text {, } \\
& K_{\widetilde{\mathbf{D}}_{4}}=\left(\begin{array}{cccc}
1 & 0 & 0 & 0 \\
2 & 1 & -1 & 2 \\
-4 & 0 & 3 & -4 \\
-2 & 0 & 1 & -1
\end{array}\right), \quad K_{\widetilde{\mathbf{B}}_{4}}=\left(\begin{array}{cccc}
-1 & -2 & 1 & -2 \\
2 & 3 & -1 & 2 \\
-4 & -4 & 3 & -4 \\
-2 & -2 & 1 & -1
\end{array}\right) \text {, }
\end{aligned}
$$

respectively. The resulting monodromy matrices are all $S L(4, \mathbb{Z})$ similar to $K_{\mathbf{A}}$ and therefore correspond to irreducible singular fibers of a new collection,

$$
\mathbf{A}^{8} \widetilde{\mathbf{B}}_{1} \widetilde{\mathbf{C}}_{1} \mathbf{B}_{2} \mathbf{C}_{2} \widetilde{\mathbf{B}}_{3} \widetilde{\mathbf{D}}_{3} \widetilde{\mathbf{B}}_{4} \widetilde{\mathbf{D}}_{4} .
$$

The similarity transformation is easy to see in the case of $K_{\widetilde{\mathbf{B}}_{1}}$ and $K_{\widetilde{\mathbf{C}}_{1}}$, since these are identical to the matrices $K_{\mathbf{B}_{1}}$ and $K_{\mathbf{C}_{1}}$ of the $(m, n)=(1,2)$ case. For the remaining $K_{\tilde{\mathbf{X}}}$, an explicit choice of matrices realizing the similarity transformation $K_{\widetilde{\mathbf{X}}}=S_{\widetilde{\mathbf{x}}} K_{\mathbf{A}} S_{\widetilde{\mathbf{x}}}^{-1}$ is

$$
\begin{aligned}
S_{\widetilde{\mathbf{D}}_{3}}=\left(\begin{array}{cccc}
0 & 0 & 1 & 0 \\
1 & 0 & 0 & 1 \\
0 & 1 & 2 & 0 \\
-1 & 0 & 0 & 0
\end{array}\right), & S_{\widetilde{\mathbf{B}}_{3}}=\left(\begin{array}{cccc}
1 & 0 & 0 & 1 \\
-1 & 0 & -1 & -1 \\
0 & -1 & -2 & 0 \\
1 & 0 & 0 & 0
\end{array}\right), \\
S_{\widetilde{\mathbf{D}}_{4}} & =\left(\begin{array}{cccc}
0 & 0 & -1 & 0 \\
1 & 0 & 0 & 0 \\
-2 & 1 & -2 & -2 \\
-1 & 0 & 0 & -1
\end{array}\right), \quad S_{\widetilde{\mathbf{B}}_{4}}=\left(\begin{array}{cccc}
1 & 0 & 0 & 0 \\
-1 & 0 & 1 & 0 \\
2 & -1 & 2 & 2 \\
1 & 0 & 0 & 1
\end{array}\right),
\end{aligned}
$$

The vanishing cycles are given by $(1,-1,2,0)$ and $(1,1,-2,0)$ for $\widetilde{\mathbf{B}}_{1}$ and $\widetilde{\mathbf{C}}_{1}$, respectively, and by the first column of the corresponding matrix in Eq. (3.60) for $\widetilde{\mathbf{B}}_{3}, \widetilde{\mathbf{D}}_{3}, \widetilde{\mathbf{B}}_{3}$, and $\widetilde{\mathbf{D}}_{3}$.

From the rescaling of $y^{3}$, the Hodge form on the quotient (obtained from $2 \omega_{\text {old }}$ ) is

$$
\omega_{\text {new }}=2 d y^{1} \wedge d y^{2}+d y^{3} \wedge d y^{4}
$$


of polarization $(2,1)$. Since the $\mathbb{Z}_{2}$ is freely acting, the quotient is again a Calabi-Yau manifold, with trivial $\mathrm{MW}_{\text {tor }}$ and $\pi_{1}=\mathbb{Z}_{2}$. From these properties, we see that it is a new Calabi-Yau manifold, distinct from the set of $\mathcal{X}_{m, n}$ dual to $T^{6} / \mathbb{Z}_{2}$.

In this example, the $\mathbb{Z}_{2}$ action on a reducible (pairwise coalesced) fiber exchanges the two components, leaving an irreducible fiber. This is case 2 below. More generally, there are three possibilities for the action of an element of $\mathrm{MW}_{\text {tor }}$ on a reducible fiber:

1. On each singular elliptic curve of the reducible fiber, the isometry acts freely by translation. In this case, there is no change in the type of the reducible fiber. The monodromy matrix factorizes into the same number of irreducible matrices (each similar to $K_{\mathbf{A}}$ ) before and after quotienting.

2. The isometry permutes the components of the reducible fiber. In this case, if there is a single orbit, then the fiber becomes irreducible after quotienting and the monodromy also becomes irreducible (similar to $K_{\mathbf{A}}$ ). If there is more than one orbit, then there is one irreducible component for each orbit.

3. The isometry is along the vanishing cycle (i.e., each point of the singular locus of the reducible fiber is a fixed point of the isometry). In this case, the singular fiber becomes "more singular," i.e., reducible into more components after quotienting, and the monodromy matrix factorizes into more irreducible factors after quotienting than before.

\section{Algebraic construction in the principally polarized case}

In this section, we change gears and provide a second construction of the type IIA CalabiYau geometry dual to $T^{6} / \mathbb{Z}_{2}$, this time taking an explicit algebro-geometric approach and focusing on the principally polarized case $m, n=1,1$. Following Saito [41, 42, 43], we construct a principally polarized abelian surface fibration over $\mathbb{P}^{1}$ as the relative Jacobian of a genus-2 fibered surface $S$. We show that it satisfies all of the required properties to be the Calabi Yau manifold $\mathcal{X}_{1,1}$ described in the previous section. By Wall's theorem [35] and

its extension due to Z̆ubr, a Calabi-Yau manifold is determined up to homotopy type by its Hodge numbers, second Chern class, and intersection numbers [35, 54]. We show that all of these quantities agree with those of $\mathcal{X}_{1,1}$, and in addition, compute the Mordell-Weil lattice from this perspective.

The construction begins with a pencil of genus- 2 curves - that is, with surface $S$, fibered over $\mathbb{P}^{1}$ via a projection map $\rho: S \rightarrow \mathbb{P}^{1}$, whose generic fibers $C_{p}=\rho^{-1}(p)$ are smooth curves of genus 2 .

Associated to each fiber $C_{p}$ is its Jacobian $\mathcal{J}_{C_{p}} \cong T^{4}$, which is a principally polarized abelian surface. (See App. F for a review of complex curves and their Jacobians.) The 
relative Jacobian $\mathcal{J}_{S / \mathbb{P}^{1}}$ is an abelian surface fibration over $\mathbb{P}^{1}$, obtained from $S$ by replacing each fiber $C_{p}$ with $\mathcal{J}_{C_{p}}$. For the appropriate choice of $S$, we show that $\mathcal{J}_{S / \mathbb{P}^{1}}$ is the desired Calabi-Yau 3-fold.

\subsection{The surface $S$}

Our choice of $S$ is as follows. Let $L^{2}$ be a line bundle of degree $(6,2)$ over $\mathbb{P}_{s, t}^{1} \times \mathbb{P}_{u, v}^{1}$, and let $f(s, t ; u, v)$ be a homogeneous polynomial of degree $(6,2)$, so that $f$ defines a section of $L^{2}$. Then, $f^{1 / 2}$ defines a 2 -fold section of the line bundle $L$ of degree $(3,1)$, branched over the curve $B=\{f=0\}$ in $\mathbb{P}^{1} \times \mathbb{P}^{1}$. I.e., it is a double cover of $\mathbb{P}^{1} \times \mathbb{P}^{1}$ branched over $B$. This double cover is the desired surface $S$.

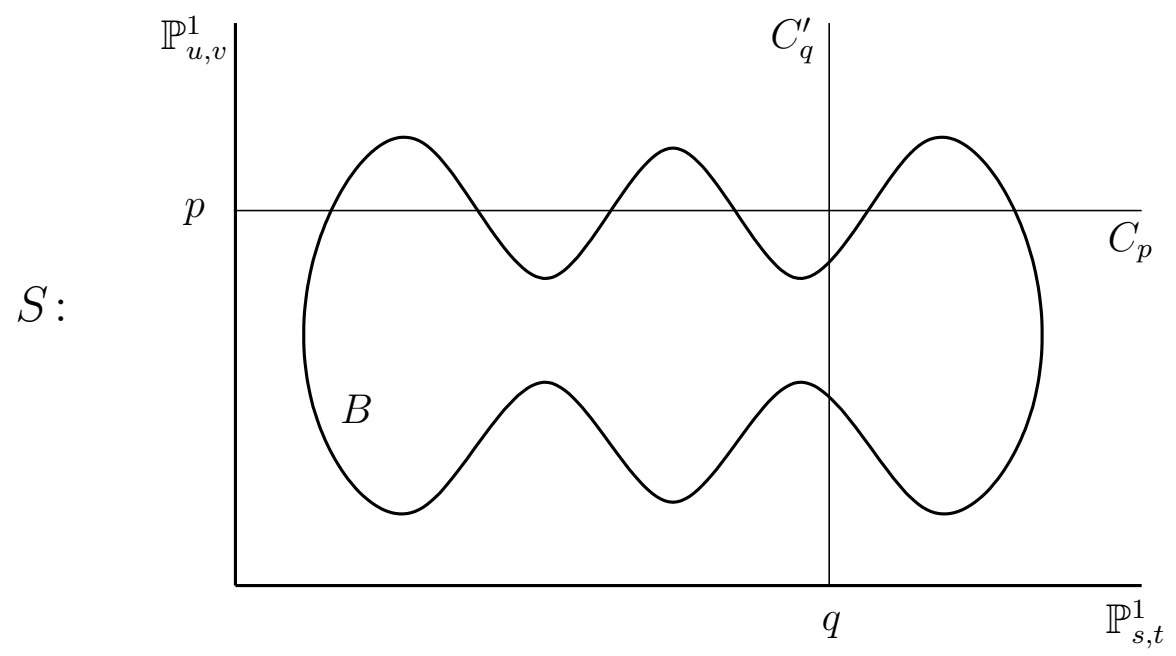

Figure 13: The surface $S$ is a double cover of $\mathbb{P}_{s, t}^{1} \times \mathbb{P}_{u, v}^{1}$ branched over a degree $(6,2)$ curve $B$. The fiber $C_{p}$ over a point $p \in \mathbb{P}_{u, v}^{1}$ is a double cover of $\mathbb{P}_{u, v}^{1}$ with 6 branch points (genus-2), and the fiber $C_{q}^{\prime}$ over a point $q \in \mathbb{P}_{s, t}^{1}$ is a double cover of $\mathbb{P}_{u, v}^{1}$ with 2 branch points (genus- 0 ).

The surface $S$ can be viewed as a fibration in at least two ways, corresponding to the natural projections $\rho: S \rightarrow \mathbb{P}_{u, v}^{1}$ and $\rho^{\prime}: S \rightarrow \mathbb{P}_{s, t}^{1}$. In the first case, the fiber $C_{p}$ over a generic point $p=[u, v]$ is a double cover of $\mathbb{P}_{s, t}^{1}$ branched over the 6 points $[s, t]$ where $f(s, t ; u, v)=0$. In this case, $g\left(C_{p}\right)=2 .{ }^{28}$ This is the desired genus-2 fibration. In the second case, the fiber $C_{q}^{\prime}$ over a generic point $q=[s, t]$ is a double cover of $\mathbb{P}_{u, v}^{1}$ branched over 2 points, which is a $\mathbb{P}^{1}$.

\footnotetext{
${ }^{28}$ Recall that an elliptic curve $\left(T^{2}\right.$, with $\left.g=1\right)$ is a double cover of $\mathbb{P}^{1}$ branched over four points. Likewise, a genus $g \geq 1$ curve is a double cover of $\mathbb{P}^{1}$ branched over $2 g+2$ points.
} 


\section{Singular fibers of $S$}

To determine the number of singular fibers of the genus-2 fibration $C \rightarrow S \stackrel{\rho}{\rightarrow} \mathbb{P}_{u, v}^{1}$, we note that

$$
\begin{aligned}
\chi(S) & =\chi(C) \chi\left(\mathbb{P}^{1}\right)+n_{\text {sing }}\left(\chi\left(C_{\text {sing }}\right)-\chi(C)\right) \\
& =(-2)(2)+n_{\text {sing }}(1)=n_{\text {sing }}-4,
\end{aligned}
$$

assuming that all $n_{\text {sing }}$ singular fibers are irreducible. ${ }^{29}$ On the other hand, since $S$ is the double cover of $\mathbb{P}^{1} \times \mathbb{P}^{1}$ ramified over $B,{ }^{30}$

$$
\begin{aligned}
\chi(S) & =2 \chi\left(\mathbb{P}^{1} \times \mathbb{P}^{1}\right)-\chi(B)=2 \chi\left(\mathbb{P}^{1}\right)^{2}-\chi(B) \\
& =2(2)^{2}-(-8)=16 .
\end{aligned}
$$

Equating the two expressions, we find that $n_{\text {sing }}=20$. In the same manner, it can be shown that there are 12 singular fibers of the fibration $C^{\prime} \rightarrow S \stackrel{\rho^{\prime}}{\rightarrow} \mathbb{P}_{s, t}^{1}$.

\section{Sections $\mathbb{P}^{1} \hookrightarrow S$}

A section $\ell$ of the genus- 2 fibration is a rational curve $\ell \subset S$ that projects 1 -to-1 to $\mathbb{P}_{u, v}^{1}$ and therefore intersects each fiber in exactly one point. There are 24 such curves. To identify them, first consider the projection $\rho^{\prime}: S \rightarrow \mathbb{P}_{s, t}$. The generic fiber $C^{\prime}$ is a smooth $\mathbb{P}^{1}$ that can be thought of as a double cover of $\mathbb{P}_{u, v}^{1}$ ramified at its two points of intersection with $B$. So, it projects 2 -to- 1 rather than 1 -to- $1 \mathbb{P}_{u, v}^{1}$ and intersects each genus- 2 curve $C$ (i.e., locus of fixed $[u, v])$ twice. However, on 12 singular fibers, the two ramification points coincide, and the fiber is a nodal $\mathbb{P}^{1}$ : it consists of two $\mathbb{P}^{1} \mathrm{~s}$ intersecting in a point, one on each branch of the double cover. Each of the two $\mathbb{P}^{1}$ s projects 1 -to- 1 to $\mathbb{P}_{u, v}^{1}$ and intersects each genus-2 curve once. This gives $2 \times 12=24$ sections of the genus- 2 fibration, denoted by $\ell_{I}, \ell_{I}^{\prime}$, for $I=1, \ldots, 12$. In fact, these are the only sections. Indeed, the image in $\mathbb{P}_{s, t}^{1} \times \mathbb{P}_{u, v}^{1}$ of any section is a section of $\mathbb{P}_{s, t}^{1} \times \mathbb{P}_{u, v}^{1}$ over $\mathbb{P}_{u, v}^{1}$, so it must be a copy of $\mathbb{P}_{s, t}^{1}$. But away from the 12 reducible fibers, the inverse image in $S$ of a copy of $\mathbb{P}_{s, t}^{1}$ is an irreducible two-section, so it does not contain any sections.

\section{Cohomology of $S$}

The cohomology of $S$ is easily calculated from the Leray spectral sequence for the projection $\pi: S \rightarrow \mathbb{P}_{s, t}^{1}$. (See App. $\mathrm{H}$ for background on direct images and their relation to Leray

\footnotetext{
${ }^{29}$ That is, we assume that $C_{\text {sing }}$ is a genus- 2 curve in which a single 1-cycle has contracted, or equivalently, an elliptic curve with 2 points identified. This gives a rational double point singularity of $C_{\text {sing }}$.

${ }^{30}$ Note that $B$ is of genus 5 . This is a special case of the result that $g=(\alpha-1)(\beta-1)$ for a degree $(\alpha, \beta)$ curve in $\mathbb{P}^{1} \times \mathbb{P}^{1}$ (cf. App. G).
} 
spectral sequences.) As we have just seen, the generic fiber is a $\mathbb{P}^{1}$, while precisely 12 fibers are reducible, $\mathbb{P}^{1} \cup \mathbb{P}^{1}$. We see that the derived image sheaves are:

$$
R^{0}=\mathbb{Z}, \quad R^{1}=0
$$

and $R^{2}$ is the direct sum of $\mathbb{Z}$ and 12 skyscraper sheaves supported at the 12 singular fibers. It follows that the Leray spectral sequence degenerates at $E^{2}$, and:

$$
h^{0}(S)=1, \quad h^{1}(S)=0, \quad h^{2}(S)=h^{1,1}(S)=2+12=14 .
$$

Note that

$$
\ell_{I}+\ell_{I}^{\prime}=C^{\prime} \quad \text { in } H_{2}(S, \mathbb{Z})
$$

where $C^{\prime}$ is the class of the generic $\mathbb{P}^{1}$ fiber over $\mathbb{P}_{s, t}^{1}$. Therefore, the sections $\ell_{I}, \ell_{I}^{\prime}$ of the genus-2 fibration span a 13 dimensional sublattice of $H_{2}(S, \mathbb{Z})$. Adding the generic genus-2 fiber $C$ gives the full lattice $H_{2}(S, \mathbb{Z})$.

\subsection{The 3-fold $\mathcal{X}$}

Starting from the genus-2 fibration $C \rightarrow S \stackrel{\rho}{\rightarrow} \mathbb{P}^{1}$, we define a 3 -fold $\mathcal{X}$ as the relative Jacobian,

$$
\mathcal{X}=\mathcal{J}_{S / \mathbb{P}^{1}}=\operatorname{Pic}^{0}\left(S / \mathbb{P}^{1}\right) .
$$

As already mentioned, this means that $\mathcal{X}$ is obtained from $S$ by replacing the genus- 2 fiber $C_{p}=\rho^{-1}(p)$ over each generic point $p$ with its Jacobian abelian surface $A_{p}=\mathcal{J}_{C_{p}}$. Singular fibers are replaced by the compactifications of their Jacobians. Let $\pi$ denote the corresponding projection map. Then, $\mathcal{X}$ is an abelian surface fibration $A \rightarrow \mathcal{X} \stackrel{\pi}{\rightarrow} \mathbb{P}^{1}$.

\section{Number and type of singular fibers of $\mathcal{X}$}

Like $S$, the 3 -fold $\mathcal{X}$ has 20 singular fibers, in agreement with the number of singular fibers of $\mathcal{X}_{1,1}$ in Sec. 2. Each is topologically of $\mathrm{I}_{1} \times T^{2}$ type. However, the complex structure does not in general respect this factorization. Instead, the singular fiber should be viewed as the $\mathbb{P}^{1}$ bundle $\mathbb{P}^{1}(\mathcal{O}(p) \oplus \mathcal{O}(q))$ over an elliptic curve $E$, with the zero section $[0, *]$ glued to the section at infinity $[*, 0]$. Here $p$ and $q$ are two points on $E .^{31}$ This leaves a unique section $E_{0} \cong E_{\infty}$. This elliptic curve is the singular locus of the singular fiber.

To derive this form for the singular fibers of the $\mathcal{X}$, let us first consider the singular fibers of $S$ and then find their compactified Jacobians. Recall that the generic $\mathrm{I}_{1}$ degeneration of an elliptic fibration can be viewed as a $\mathbb{P}^{1}$ with two points identified; these are the $2 g+2=2$

\footnotetext{
${ }^{31}$ When $p=q$, the fiber factorizes as $E$ times $\mathrm{I}_{1}$, where the $\mathrm{I}_{1}$ is realized as $\mathbb{P}^{1}$ with the points 0 and $\infty$ identified. But when $p \neq q$ we instead identify $\infty \in \mathbb{P}_{r}^{1}$ in the fiber at each point $r \in E$ with $\infty \in \mathbb{P}_{r+(q-p)}^{1}$ in a different fiber at the shifted point $r+(q-p) \in E$.
} 
branch points in the presentation of $\mathbb{P}^{1}$ as branched double cover of $\mathbb{P}^{1}$. Likewise, a genus-2 curve $C$ is a branched double cover of $\mathbb{P}^{1}$ with 6 branch points, so the generic singularity is one in which 2 of these points coincide. It can be viewed as an elliptic curve $E$ with two points $p$ and $q$ identified

$$
C \cong E /\{p \sim q\} \quad \text { (generic degeneration of the genus-2 curve } C \text { ). }
$$

The normalization map $\nu: E \rightarrow C$ identifies the points $p$ and $q$.

A line bundle on $C$ pulls back via the normalization map $\nu$ to a line bundle on $E$. In fact, specifying a line bundle on $C$ is the same as specifying a line bundle $L$ on $E$ together with a gluing of the fibers of $L$ at $p, q$. The set of these gluings is a copy of the group of isomorphisms from the line $C$ to itself, which is given by the multiplicative group $C^{*}$. It follows that $\operatorname{Pic}(C)$ is an extension of $\operatorname{Pic}(E)$ by $C^{*}$. In order to compactify, we must allow torsion free sheaves on $C$ that are not necessarily line bundles. The effect is to replace each fiber $C^{*}$ by its compactification $\mathbb{P}^{1}$. Note what happens when the gluing parameter $t \in C^{*}$ goes to 0 : the map from $L_{p}$ to $L_{q}$, which is an isomorphism for most $t$, becomes the 0 map as $t \rightarrow 0$. The result is the torsion free sheaf $\nu_{*}\left(L \otimes \mathcal{O}_{E}(-p)\right)$. Its fiber at the singular point has rank 2 rather than 1 , and consists of the direct sum of the fiber of $L \otimes \mathcal{O}_{E}(-p)$ at $p$ with the fiber of $L$ at $q$. On the other hand, when the gluing parameter $t \in C^{*}$ goes to $\infty$, the inverse map from $L_{q}$ to $L_{p}$, which is an isomorphism for most $t$, becomes the 0 map as $t \rightarrow \infty$. The result is now the torsion free sheaf $\nu_{*}\left(L \otimes \mathcal{O}_{E}(-q)\right)$. Its fiber at the singular point consists of the direct sum of the fiber of $L$ at $p$ with the fiber of $L \otimes \mathcal{O}_{E}(-q)$ at $q$. In other words, the 0 and $\infty$ sections of the $\mathbb{P}^{1}$ fibration over $\operatorname{Pic}(E)$ are glued to each other, but the gluing is not the obvious one: it involves a shift of $\mathcal{O}_{E}(p-q)$. This is summarized in the following diagram:

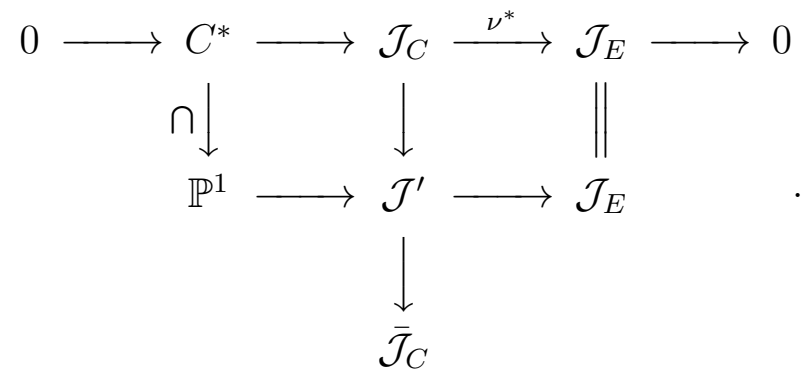

\section{Hodge numbers}

The number of complex structure deformations of $S$ is the choice of degree $(6,2)$ polynomial $f(s, t ; u, v)$ modulo equivalences:

$$
h^{1,1}(S)=(6+1)(2+1)-(1 \text { overall rescaling })-\left(3+3 \text { from } S L(2, \mathbb{C})^{2}\right)=14 .
$$

The complex structure deformations of $\mathcal{X}$ are in 1-to-1 correspondence with those of $S$. Therefore, $h^{2,1}(\mathcal{X})=14$. But we have just seen that every fiber of $\mathcal{X}$ over $\mathbb{P}^{1}$ is either an 
abelian surface, which is topologically $T^{4}$, or it is singular, in which case it is topologically $T^{2}$ times a nodal curve. Either way, the Euler characteristics of all fibers vanish. It follows that the Euler characteristic of $\mathcal{X}$ vanishes as well, so $h^{1,1}=h^{2,1}=14$. The Hodge numbers of $\mathcal{X}$ agree with those $\mathcal{X}_{1,1}$.

\section{The Calabi-Yau condition}

We wish to show that the manifold $\mathcal{X}$ has trivial canonical bundle. Consider any $\mathbb{P}^{1}$ section of the abelian fibration $\mathcal{X}$. By the adjunction formula,

$$
K_{\mathcal{X} \mid \mathbb{P}^{1}}=K_{\mathbb{P}^{1}} \otimes \operatorname{det}\left(N_{\mathbb{P}^{1}}^{*}\right)=\mathcal{O}_{\mathbb{P}^{1}}(-2) \otimes \operatorname{det}\left(N_{\mathbb{P}^{1}}^{*}\right)
$$

where $N_{\mathbb{P}^{1}}^{*}$ is the conormal bundle to $\mathbb{P}^{1}$ in $\mathcal{X}$. Therefore, $\mathcal{X}$ is a Calabi-Yau manifold if $\operatorname{det}\left(N_{\mathbb{P}^{1}}^{*}\right)=\mathcal{O}_{\mathbb{P}^{1}}(2)$. To compute $N_{\mathbb{P}^{1}}^{*}$, we note that

$$
N_{\mathbb{P}^{1}}^{*}=\rho_{*} K_{S / \mathbb{P}^{1}}
$$

where $\rho_{*}$ is the direct image functor ${ }^{32}$ of the projection map $\rho: S \rightarrow \mathbb{P}_{u, v}^{1}$. In App. I, it is shown that this gives $N_{\mathbb{P}^{1}}=\mathcal{O}_{\mathbb{P}^{1}}(-1) \oplus \mathcal{O}_{\mathbb{P}^{1}}(-1)$, from which the desired result follows.

\subsection{Checks}

\subsubsection{Intersection numbers}

In App. J, it is shown that to each of the 24 sections $\ell_{I}, \ell_{I}^{\prime}$, for $I=1, \ldots, 12$, we can associate a theta surface $\Theta_{I}$ or $\Theta_{I}^{\prime} \in \mathcal{X}$. The theta surfaces are embeddings of $S$ in the Calabi-Yau threefold $\mathcal{X}$. They satisfy homology relations analogous to those of Eq. 4.5,

$$
\Theta_{I}+\Theta_{I}^{\prime}=D \quad \text { in } H_{4}(\mathcal{X}, \mathbb{Z})
$$

where $D$ is independent of $I$.

The theta surfaces together with generic abelian fiber $A$ form a basis of $H_{4}(\mathcal{X}, \mathbb{Z})$. Their double and triple intersections are computed in App. J. The result for the triple intersections of theta surfaces is

$$
\begin{aligned}
& \Theta_{I} \cdot \Theta_{J} \cdot \Theta_{K}=-1, \\
& \Theta_{I} \cdot \Theta_{J} \cdot \Theta_{J}=\Theta_{I} \cdot \Theta_{J}^{\prime} \cdot \Theta_{J}^{\prime}=-2, \\
& \Theta_{I} \cdot \Theta_{I} \cdot \Theta_{I}^{\prime}=\Theta_{I} \cdot \Theta_{J} \cdot \Theta_{J}^{\prime}=0, \\
& \Theta_{I} \cdot \Theta_{I} \cdot \Theta_{I}=-4,
\end{aligned}
$$

\footnotetext{
${ }^{32}$ See App. $\mathrm{H}$ for background on the direct image $\rho_{*}$ and higher direct images (derived functors) $R^{i} \rho_{*}$.
} 
for $I, J, K$ distinct, together with equations obtained from these by exchange of $\Theta$ and $\Theta^{\prime}$. For triple intersections in which $A$ appears, we have $A^{2}=0$, and

$$
A \cdot \Theta_{I} \cdot \Theta_{J}=A \cdot \Theta_{I} \cdot \Theta_{J}^{\prime}=A \cdot \Theta_{I}^{\prime} \cdot \Theta_{J}^{\prime}=2,
$$

for all $I, J$, not necessarily distinct.

With the identifications,

$$
\mathcal{E}_{I}=\left(\Theta_{I}-\Theta_{I}^{\prime}\right) / 2, \quad H=\left(\Theta_{I}+\Theta_{I}^{\prime}\right) / 2+A / 6,
$$

these intersections precisely match the result (3.8) obtained by classical supergravity duality in Ref. [46]. It is important to confirm that the integrality matches as well. The factors of $1 / 2$ are exactly as expected. The fact that $\mathcal{E}_{I}$ is half of an integer divisor is equivalent to the statement in the $T^{6} / \mathbb{Z}_{2}$ dual that a string stretched between a single D3-brane and its image represents half of a root of $S O(2 M)$ (cf. Sec. 2.4). The factor of $1 / 2$ in $H$ can be understood in a similar way. The appearance of the $A / 6$ term in $H$ is more subtle and requires a careful definition of warped volume in the $T^{6} / \mathbb{Z}_{2}$ dual to justify its appearance. A proper treatment of this subtlety is an essential ingredient of the analysis of duality map between D3 instantons and worldsheet instantons under investigation in Ref. [25], to which the reader is referred for further details. ${ }^{33}$

\subsubsection{Second Chern class $c_{2}(\mathcal{X})$}

The second Chern class $c_{2}(\mathcal{X})$ is the sum of the 20 elliptic curves $E_{i}$ that are singular loci of the singular compactified Jacobians. Its intersection with a theta surface, for any of the 24 possible choices $\Theta_{I}$ or $\Theta_{I}^{\prime}$, is 20, and its intersection with the generic abelian fiber $A$ is zero. Thus, $H \cdot c_{2}=20$ for $H$, as given by Eq. (3.9).

To derive $c_{2}$, we note that the normal bundle sequence has a very simple modification: the vertical subsheaf of the tangent bundle is still a line subbundle, which is still $\pi^{*}$ of the normal bundle to $\mathbb{P}^{1}$ in $\mathcal{X}$ (for any $\mathbb{P}^{1}$ section of the fibration); the horizontal piece is still $\pi^{*} T_{\mathbb{P}^{1}}$, but the map now is not surjective - instead there is a cokernel which is the structure sheaf of the 20 elliptic curves.

$$
\left.0 \rightarrow N_{\mathbb{P}^{1}} \rightarrow T_{\mathcal{X}} \rightarrow \pi^{*} T_{\mathbb{P}^{1}} \rightarrow \pi^{*} T_{\mathbb{P}^{1}}\right|_{\bigcup_{i=1}^{20} E_{i}} \rightarrow 0
$$

Here, $\Delta=\left\{p_{1}, \ldots, p_{20}\right\}$ is the discriminant locus of the genus-2 fibration $\rho: S \rightarrow \mathbb{P}^{1}$, and the elliptic curves $E_{i} \in \pi^{-1}\left(p_{i}\right)$ are the singular loci of the degenerate fibers of $\pi: \mathcal{X} \rightarrow \mathbb{P}^{1}$.

\footnotetext{
${ }^{33}$ Note that Ref. [46] missed the subtlety responsible for the $A / 6$ term in $H$. There, $H$ was naively identified with a single theta surface, based on the fact that $A \cdot H$ gives the homology class of $C$. However, this intersection is preserved when $H$ is shifted by a multiple of $A$ or replaced by a weighted sum of theta surfaces of total weight 1. Indeed, $H$ in Eq. (4.15) differs from a single theta surface in exactly these two ways.
} 
Note that in the discussion of the first Chern class (Calabi-Yau condition) above in Sec. 4.2, there was no special contribution from singular fibers. This is intuitively clear. The $\mathbb{P}^{1}$ sections intersect singular fibers at smooth points, so the singular fibers should not affect $c_{1}$.

\subsubsection{Mordell-Weil lattice: $D_{12}^{-}$}

As discussed in Sec. 2 and App. B, the Mordell-Weil group $\operatorname{MW}(\mathcal{X})$ is the group of rational sections of the Abelian surface fibration $\mathcal{X}$, and the Mordell-Weil lattice $\mathrm{MW}(\mathcal{X}) / \mathrm{MW}_{\text {tor }}(\mathcal{X})$ is the lattice of sections modulo torsion. To determine this lattice, we would like to relate the sections of $\mathcal{X}$ to those of the genus-2 fibration $S$. The sections of $\mathcal{X}$ and $S$ do not quite encode the same data. In particular, the sections of the abelian fibration $\mathcal{X}$ form a rank 12 lattice, while those of $S$ form a finite set (just the 12 pairs $\ell_{I}, \ell_{I}^{\prime}$ of Sec. 4.1). However, given a choice of section $\ell_{0}$ to play the role of zero section, we can map each of the 24 sections of $S$ to a section of $\mathcal{X}$, as we now show. This allows us to describe the Mordell-Weil lattice of $\mathcal{X}$ as a sublattice of $H_{2}(S, \mathbb{Z})$.

The 24 sections $\ell_{I}, \ell_{I}^{\prime} \subset S$ can be thought of as elements of $\Gamma\left(\mathbb{P}^{1}, \operatorname{Pic}^{1}\left(S / \mathbb{P}^{1}\right)\right) .{ }^{34}$ Since $\mathcal{X}=\operatorname{Pic}^{0}\left(S / \mathbb{P}^{1}\right)$, we similarly have

$$
\operatorname{MW}(\mathcal{X})=\Gamma\left(\mathbb{P}^{1}, \operatorname{Pic}^{0}\left(S / \mathbb{P}^{1}\right)\right) .
$$

To relate the two, we note that $\operatorname{Pic}^{1}\left(S / \mathbb{P}^{1}\right) \cong \operatorname{Pic}^{0}\left(S / \mathbb{P}^{1}\right)$ via a noncanonical isomorphism,

$$
\operatorname{Pic}^{1}\left(S / \mathbb{P}^{1}\right) \stackrel{\otimes\left[\ell_{0}\right]^{-1}}{\longrightarrow} \operatorname{Pic}^{0}\left(S / \mathbb{P}^{1}\right), \quad \ell \mapsto \ell-\ell_{0},
$$

where $\ell_{0} \in\left\{\ell_{I}, \ell_{I}^{\prime}\right\}$. The isomorphism depends on the choice of which of the 24 sections $\ell_{I}, \ell_{I}^{\prime}$ maps to the zero section of $\mathcal{X}$.

This isomorphism endows $\operatorname{Pic}^{1}\left(S / \mathbb{P}^{1}\right)$ with the structure of an abelian group and allows us to relate the Mordell-Weil lattice of $\mathcal{X}$ to the Néron-Severi lattice ${ }^{35}$ of $S$ via

$$
\operatorname{MW}(\mathcal{X}) \cong K^{\perp} \subset \mathrm{NS}(S)
$$

where $K$ is spanned by $\ell_{0}$, the generic genus-2 fiber $C$, and the components of reducible fibers (in the case that there exist special fibers with multiple components). Here, the intersection pairing on $S$ is used to define both the orthogonal complement $K^{\perp}$ and its height pairing.

\footnotetext{
${ }^{34}$ See App. F for the definition of $\operatorname{Pic}^{n}\left(S / \mathbb{P}^{1}\right)$ and further elaboration of this statement.

${ }^{35}$ The Néron-Severi lattice is roughly the same as the algebraic (i.e., 1,1$)$ part of $H_{2}(S, \mathbb{Z})$. There are several closely related definitions of equivalence classes of divisors, for example, homological equivalence, linear equivalence (same line bundle) and numerical equivalence (same intersections). The Néron-Severi lattice is the lattice of algebraic equivalence classes of divisors, however, we will not need the technical definition of algebraic equivalence here, since on a projective variety, homological equivalence of divisors is equivalent to algebraic equivalence. (See Ref. [28], p. 462.) In this case, Néron-Severi lattice is $\operatorname{NS}(S) \cong$ $H^{1,1}(S) \cap H^{2}(S, \mathbb{Z}) \cong \operatorname{Pic}(S) / \operatorname{Pic}^{0}(S)$.
} 
Let us assume for simplicity that there are no reducible fibers. Then the Mordell-Weil lattice is just the orthogonal complement of $\ell_{0}$ and $C$ in $\operatorname{NS}(S)$. From the intersections

$$
\ell \cdot \ell=-1, \quad \ell \cdot C=1, \quad C \cdot C=0,
$$

for any section $\ell$ of $S$, the map from $\operatorname{NS}(S)$ to $\operatorname{MW}(\mathcal{X})$ is

$$
v \mapsto v^{\perp}=v-(v \cdot C) \ell_{0}-\left(v \cdot\left(\ell_{0}+C\right)\right) C .
$$

The resulting lattice is the root lattice of $D_{12}$. To see this, choose $\ell_{0}=\ell_{12}^{\prime}$ for concreteness. Then, from

$$
\ell_{I} \cdot \ell_{J}^{\prime}=\delta_{I J}, \quad \ell_{I} \cdot \ell_{J}=\ell_{I}^{\prime} \cdot \ell_{J}^{\prime}=-\delta_{i j},
$$

we have

$$
\begin{aligned}
\ell_{I} & \mapsto \ell_{i}^{\perp}=\ell_{I}-\ell_{12^{\prime}}-C, \\
\ell_{I}^{\prime} & \mapsto \ell_{i}^{\perp}=\ell_{I}^{\prime}-\ell_{12^{\prime}}-C,
\end{aligned}
$$

for $i=1, \ldots, 11$, and

$$
\ell_{12} \mapsto \ell_{12}^{\perp}=\ell_{12}-\ell_{12}^{\prime}-2 C,
$$

with $\ell_{12}^{\prime} \mapsto \ell_{12}^{\prime \perp}=0$ and $C \mapsto C^{\perp}=0$.

The $D_{12}$ roots are $v_{I}=\ell_{I}^{\perp}-\ell_{I+1}^{\perp}$, for $I=1, \ldots, 11$ and $v_{12}=\ell_{11}^{\perp}$. In terms of the sections $\ell_{I}$ and $\ell_{I}^{\prime}$,

$$
\begin{aligned}
v_{I} & =\ell_{I}-\ell_{I+1}=\ell_{I+1}^{\prime}-\ell_{I}^{\prime}, \quad I=1, \ldots, 10, \\
v_{11} & =\ell_{11}-\ell_{12}+C \\
v_{12} & =\ell_{11}-\ell_{12}^{\prime}-C .
\end{aligned}
$$

The roots $v_{I}$ generate a 12 dimensional sublattice of $\operatorname{NS}(S)$. Their intersection matrix is minus the Cartan matrix of $D_{12}$.

\subsection{Mordell-Weil torsion and connection to other CY manifolds}

In the junction description, we have seen a number of examples in Sec. 3.5 with enhanced Mordell-Weil torsion from coalesced singular fibers. The simplest example is $\mathbb{Z}_{2}$ torsion, which arises when 4 pairs of singular fibers of $\mathcal{X}_{m, n}$ coalesce, to give 4 reducible singular fibers (of topological type $\mathrm{I}_{2} \times T^{2}$ ) and 4 elliptic curve of $A_{1}$ singularities of the threefold.

In the case of $\mathcal{X}_{1,1}$, it should be possible to reproduce the results of Sec. 3.5 in the present description in terms of the relative Jacobian of $S$. In this construction, the surface $S$ is a branched double cover of $\mathbb{P}_{s, t}^{1} \times \mathbb{P}_{u, v}^{1}$, so the singularity structure of $S$, and hence of $\mathcal{X}$, is completely determined by the degree $(6,2)$ branch curve $B$. Coalesced fibers, in the language of Sec. 3.5, arise when $B$ becomes singular in some way. For surfaces, the correspondence 
between singularities of a double cover and those of the branch curve is well understood. (See, for example, Ref. [5] Sec. III.7.) One way that $B$ can develop singularities, is if it is reducible, i.e., if its defining polynomial factorizes. Then singularities arise from intersections of different components of $B$. This is not the only way in which $B$ can develop singularities, but it will be sufficient for our purposes.

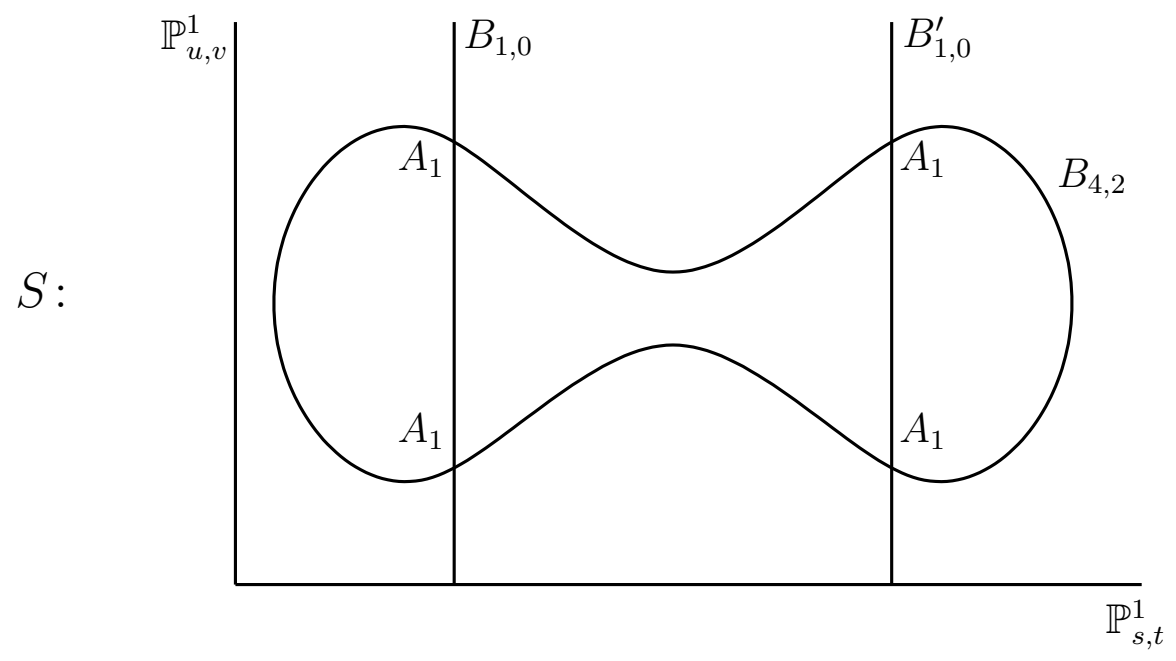

Figure 14: The surface $S$ is a double cover of $\mathbb{P}_{s, t}^{1} \times \mathbb{P}_{u, v}^{1}$ branched over $B$. When the branch curve $B$ factorizes into a degree $(4,2)$ curve $B_{4,2}$ (of genus 3 ) and two $(1,0)$ curves $B_{1,0}$ and $B_{1,0}^{\prime}$ (of genus 0 ), the difference $B_{1,0}-B_{1,0}^{\prime}$ is a $\mathbb{Z}_{2}$ torsion section of $S$.

To reproduce the simplest case of $\mathbb{Z}_{2}$ Mordell-Weil torsion, consider the factorization $(6,2)=(4,2)+(1,0)+(1,0)$, so that the branch curve $B$ has irreducible components $B_{4,2}$, $B_{1,0}$ and $B_{1,0}^{\prime}$, with the degree of each curve in $\mathbb{P}_{s, t}^{1} \times \mathbb{P}_{u, v}^{1}$ given by its subscript. Then $B$ has an $A_{1}$ rational double point at each of the four points of intersection of the components of $B$ (cf. Fig. 14), and from Ref. [5] Sec. III.7, so does the surface $S$. Note that the $(1,0)$ curves are sections of the genus- 2 fibration $S \rightarrow \mathbb{P}_{u, v}^{1}$. Moreover, $2 B_{1,0}$ and $2 B_{1,0}^{\prime}$ are each double covers of $\mathbb{P}_{u, v}^{1}$, so each is homologous to $C^{\prime}$, the genus-0 fiber of the fibrations $S \rightarrow \mathbb{P}_{s, t}^{1}$, modulo irrelevant vertical components. It follows that the difference $2\left(B_{1,0}^{\prime}-B_{1,0}^{\prime}\right)$ is trivial in the Mordell-Weil group, and $B_{1,0}^{\prime}-B_{1,0}^{\prime}$ is a $\mathbb{Z}_{2}$ torsion class. Quotienting by this isometry gives a new Calabi-Yau manifold with Hodge numbers $h^{1,1}=h^{1,2}=10$, trivial Mordell-Weil torsion, and fundamental group $\pi_{1}=\mathbb{Z}_{2}$. The $\mathbb{Z}_{2}$ action on the (resolved) reducible fibers is fixed point free, and permutes the two components.

To obtain $\mathbb{Z}_{2}{ }^{\oplus 3}$ Mordell-Weil torsion, an analogous construction goes through with the factorization $(6,2) \rightarrow(2,2)+(1,0)+(1,0)+(1,0)+(1,0)$. In this case the branch curve $B$ consists of an elliptic curve $B_{2,2}$ (cf. the genus formula of App. G), and rational curves $B_{1,0}^{i}$, for $i=1,2,3,4$, each of which is a section of the genus-2 fibration $S \rightarrow \mathbb{P}_{u, v}^{1}$. Again, $2 B_{1,0}^{i}=C^{\prime}$ in homology, modulo irrelevant vertical components on the resolution, so $\mathrm{MW}_{\text {tor }}=\mathbb{Z}_{2}{ }^{\oplus 3}$, 
generated by the three linearly independent differences of the $B_{1,0}^{i}$. This is the algebrogeometric description of the Mordell-Weil torsion in the second example of Sec. 3.5.3.

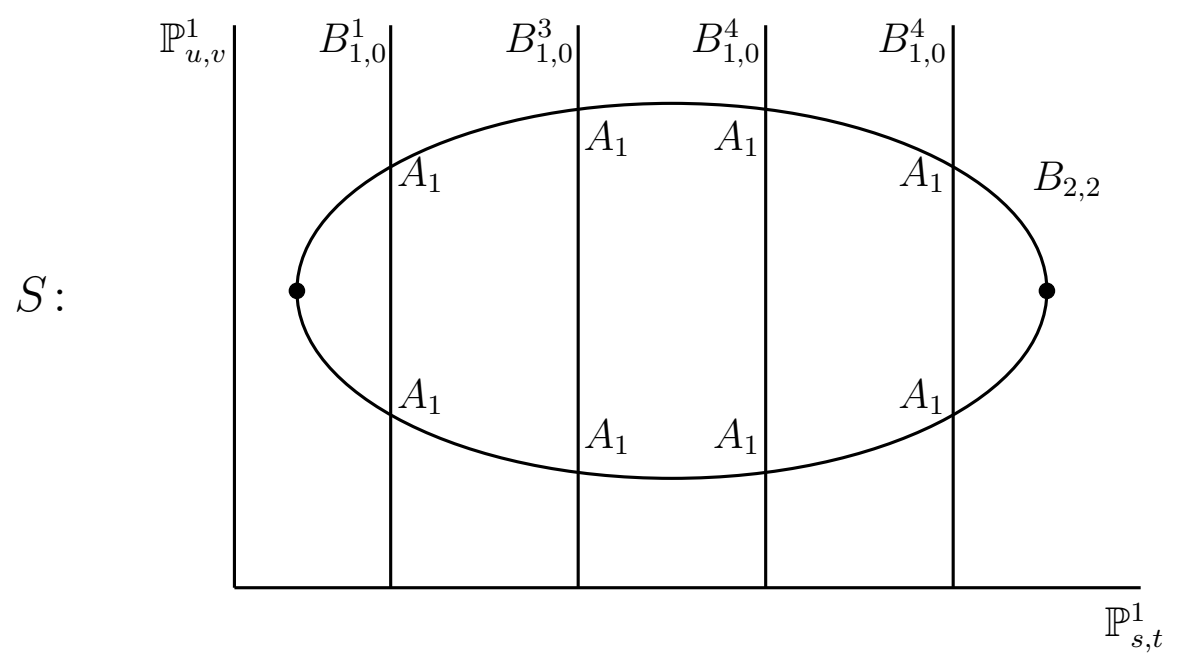

Figure 15: The surface $S$ is a double cover of $\mathbb{P}_{s, t}^{1} \times \mathbb{P}_{u, v}^{1}$ branched over $B$. When the branch curve $B$ factorizes into a degree $(2,2)$ elliptic curve $B_{2,2}$ and four $(1,0)$ rational curves $B_{1,0}^{i}$, the differences $B_{1,0}^{i}-B_{1,0}^{j}$ generate a $\left(\mathbb{Z}_{2}\right)^{\oplus 3}$ of torsion sections of $S$. Ramification points of the projection $B \rightarrow \mathbb{P}_{s, t}^{1}$ are shown in bold.

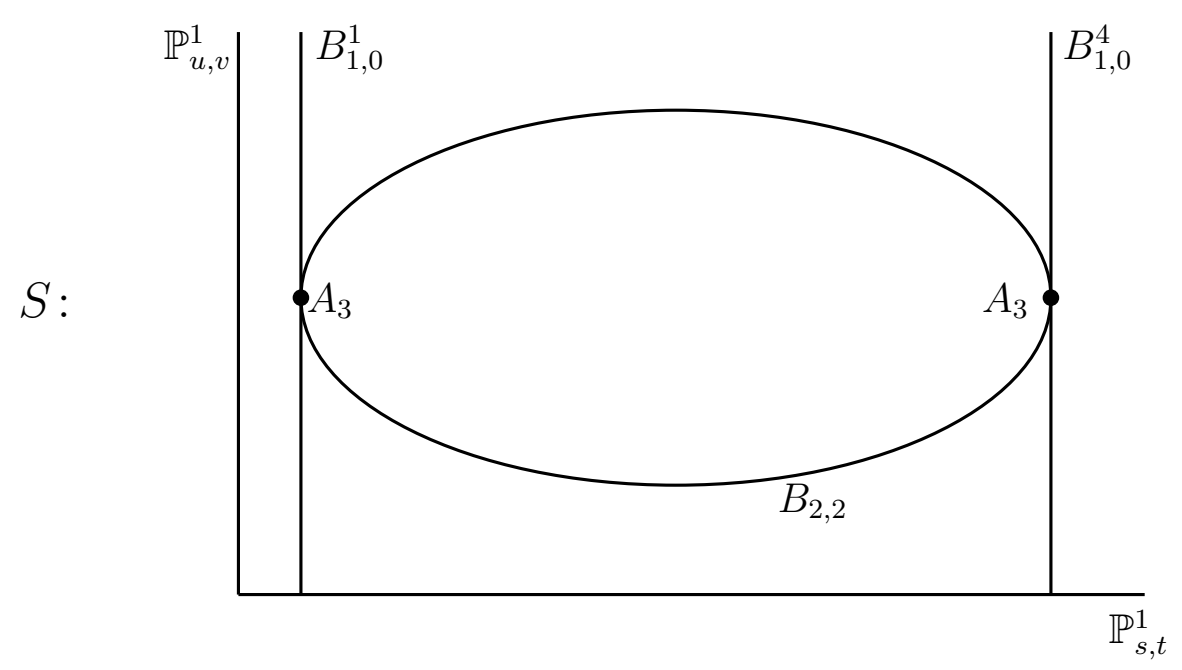

Figure 16: When the rational curve $B_{1,0}^{i}$ of Fig. 15 is placed at one of the four ramifications points of $B_{2,2} \rightarrow \mathbb{P}_{s, t}^{1}$, the resulting tacnode of the branch curve yields an $A_{3}$ singularity of the surface $S$. There are four such ramification points, but only two are visible in the schematic diagram above.

One can ask whether a further enhancement of $\mathbb{Z}_{2}^{\oplus 3}$ to $\mathbb{Z}_{4} \oplus \mathbb{Z}_{2}{ }^{\oplus 2}$ is possible, as in 
Sec. 3.5.3, by coalescing pairs of $A_{1}$ singularities of $S$ into $A_{3}$ singularities. We can indeed obtain this singularity structure by moving each $B_{1,0}^{i}$ to a ramification points of the elliptic curve $B_{2,2}$, relative to the projection $B_{2,2} \rightarrow \mathbb{P}_{s, t}^{1}{ }^{36}$ Each intersection point is then a tacnode of $B$, which from Ref. [5] Sec. III.7 gives an $A_{3}$ singularity of $S .{ }^{37}$ While this picture is tantalizing, and we have succeeded in reproducing the desired singularity structure, we have not been able to identify a $\mathbb{Z}_{4}$ isometry of the resulting reducible fibers of $S$. We leave the task of reproducing the $\mathbb{Z}_{4} \oplus \mathbb{Z}_{2}{ }^{\oplus 2}$ Mordell-Weil torsion group from the relative Jacobian construction as an open problem.

Finally, consider the remaining factorizations of the $(6,2)$ branch curve into a $(*, 2)$ component and some number of $(1,0)$ components:

$$
(5,2)+(1,0), \quad(3,2)+(1,0)^{\oplus 3}, \quad \text { and } \quad(1,2)+(1,0)^{\oplus 5} .
$$

The last two factorizations give $\mathbb{Z}_{2}{ }^{\oplus 2}$ and $\mathbb{Z}_{2}{ }^{\oplus 4}$ Mordell-Weil torsion, respectively, as obtained in Sec. 3.5.2 for $m=1$. The first factorization gives two $A_{1}$ singularities of $S$ and no MordellWeil torsion; this is exactly the result of continuing Eq. (3.45) one step further, so that the only coalesced fibers are $\left(\mathbf{B}_{1}^{2}\right)$ and $\left(\mathbf{D}_{1}^{2}\right)$.

\section{Conclusions and future directions}

We have seen two explicit constructions of the type IIA Calabi-Yau duals $\mathcal{X}_{m, n}$ of the type IIB $T^{6} / \mathbb{Z}_{2}$ orientifold:

1. A monodromy and junction based description.

2. An algebro-geometric description as the relative Jacobian of a genus-2 fibered surface $\mathrm{S}$ (in the principally polarized case, $m, n=1,1$ ).

In each case, we have computed the Mordell-Weil lattice of sections, to obtain the required $D_{M}$ lattice. From a mathematical standpoint, we have shown that the junction description provides an efficient algorithm for computing the lattice of rational sections of an abelian surface fibration in terms of tree graphs on the base - a generalization of F-theory string junction technology to $T^{4}$ fibrations. For the relative Jacobian construction, we have checked that all criteria are satisfied for the application of Wall's theorem-which classifies the threefold up to homotopy type (Hodge numbers, second Chern class, intersection numbers) - and have reproduced the $D_{12}$ Mordell-Weil lattice from this perspective.

\footnotetext{
${ }^{36}$ Only two such points are visible in the cartoon of Fig. 16, but this is a deficiency of the cartoon. We know that there are really four ramification points, exactly the number necessary for one $B_{1,0}^{i}$ to be tangent at each. (Recall that an elliptic curve (genus 1 ) is the double cover of $\mathbb{P}^{1}$ with $2 g+2=4$ branch points.)

${ }^{37}$ To apply Ref. [5], we need to use the fact that the proper transform of a tacnode is as given in the first of the diagrams listed in Sec. III.7.
} 
Applications to ongoing and future work are as follows:

D-brane instantons. Having achieved the explicit constructions, the rational curves of the $\mathcal{X}_{m, n}$ are now well understood, which lays the groundwork for the computation of worldsheet instanton corrections to the $\mathcal{N}=2$ prepotential. Worldsheet instantons wrapping $\mathbb{P}^{1}$ sections of the $T^{4}$ fibration are dual to Euclidean D3-instantons in the type IIB $T^{6} / \mathbb{Z}_{2}$ orientifold (and to D2-instantons in an intermediate type IIA dual). Therefore, they provide a duality check [25] of the modified rules for D-brane instanton corrections in warped compactification due to flux [6] and brane intersections [24] — in similar spirit to Ref. [11].

Warped KK reduction. The clasical supergravity description of $T^{6} / \mathbb{Z}_{2}$ with $\mathcal{N}=2$ flux is exactly dual to the description of a type IIA Calabi-Yau compactification in terms of an explicit, first order approximation to the Calai-Yau metric (3.10) with known harmonic forms [46]. The low lying massive modes for large Calabi-Yau base are also known. Therefore, the known procedure for ordinary Kaluza-Klein reduction to $4 \mathrm{D}$ can be re-expressed in terms of the dual variables to deduce warped $\mathrm{KK}$ reduction for $T^{6} / \mathbb{Z}_{2}$ [7]. A simpler warm-up problem applies a similar duality to deduce the warped KK reduction ansatz for the type IIA $T^{3} / \mathbb{Z}_{2}$ orientifold from the standard compactification of M-theory on a K3 surface [7].

Extended SUSY breaking by topology. In any type II Calabi-Yau compactification, extended supersymmetry is broken to $4 \mathrm{D} \mathcal{N}=2$ at the compactification scale by the CalabiYau geometry. In $T^{6} / \mathbb{Z}_{2}$, the quantized flux spontaneously breaks $\mathcal{N}=4$ supersymmetry, at a scale hierarchically lower than the compactification scale for large volume. In the dual type IIA compactification on $\mathcal{X}_{m, n}$, this gives a precise sense in which the Calabi-Yau topology spontaneously breaks $\mathcal{N}=4$ to $\mathcal{N}=2$ for large $\mathbb{P}^{1}$ base. The Calabi-Yau compactification, with $S U(3)$ Levi-Civita holonomy, can be viewed as a $S U(2)$ structure compactification, the formalism for which was worked out in Ref. [52] (and subsequent work by the Hamburg group, to appear). Applying this $S U(2)$ structure formalism to the compactification on the approximate first order metric of $\mathcal{X}_{m, n}$ is an essential step of work described in the previous paragraph, but is interesting in its own right as a concrete example of spontaneous supersymmetry breaking by topology.

Heterotic model building on new non simply connected manifolds. The Calabi-Yau duals $\mathcal{X}_{m, n}$ have $\pi_{1}=\mathbb{Z}_{n} \times \mathbb{Z}_{2}$ for $n=1,2,3,4$. Moreover, at special points in moduli space, these Calabi-Yau manifolds develop enhanced isometry groups (cf. Secs. 3.5 and 4.4), the quotients by which yield new Calabi-Yau manifolds with other fundamental groups. Since few CalabiYau manifolds with nontrivial $\pi_{1}$ are known [31], these constructions are mathematically interesting. ${ }^{38}$ In addition, they furnish a new class of compactification manifolds for Wilson line breaking of GUT groups in heterotic models [20, 43].

\footnotetext{
${ }^{38}$ From a mathematical standpoint these manifolds are also interesting in that Calabi-Yau manifolds with $T^{4}$ fibrations do not arise as hypersurfaces in $4 \mathrm{D}$ toric varieties, so only a few examples exist compared a much larger class of known K3 fibrations.
} 
Finally, let us point out two additional connections to recent work:

D(imensional) duality. Ref. [27] considered compactifications that interpolated between a compactification in the critical dimension on a Riemann surface $C$ of genus $g$ and a supercritical compactification on its Jacobian torus $\mathcal{J}_{C}$ with a timelike linear dilaton. One might expect similar compactifications to exist, connecting a subcritical compactification on $C$ to an asymptotic region with compactified on $\mathcal{J}_{C}$ with an asymptotically constant linear dilaton. Such compactifications (fibered over a $\mathbb{P}^{1}$ base) provide a context in which not only Calabi-Yau $\mathcal{X}_{1,1}$, but also the auxilliary surface $S$ of Sec. 4, is part of the physical compactification geometry.

T-fold compactifications. T-fold compactifications are nongeometric compactifications similar to compactifications on $T^{n}$ fibrations, except that the transition functions lie in the T-duality group $O(n, n ; \mathbb{Z})$ rather than its geometric subgroup $G L(n ; \mathbb{Z})[36]$. For $n=3$, the component of the T-duality group continuously connected to the identity is $S O(3,3 ; \mathbb{Z})_{+} \cong$ $S L(4 ; \mathbb{Z})$. So, in this case an $n=3 \mathrm{~T}$-fold encodes the same data as $T^{4}$ fibration over the same base [56]. Thus, the collections of $S L(4, \mathbb{Z})$ monodromies defining the Calabi-Yau manifolds $\mathcal{X}_{m, n}$ in Sec. 3.1 can alternatively be taken to define T-fold compactifications. In fact, we have already seen this as part of the duality map described in Sec. 3.1. The connection between the $T^{4}$ fibration and $n=3 \mathrm{~T}$-fold compactification is that M-theory on the former is type IIA compactified on the latter. Thus, starting from M-theory on $\mathcal{X}_{m, n}$, For one choice of M-theory circle, we recover a type IIA T-fold compactification that happens to be purely geometric - the intermediate D6/O6 orientifold with flux in the duality chain 2 of Sec. 3.1. For other choices of the M-theory circle, the T-fold is expected to be nongeometric.

\section{Acknowledgements}

We are grateful to P. Argyres, K. Becker, V. Bouchard, V. Braun, O. DeWolfe, D. Freed, K. Hori, M.-H. Saito and G. Segal for conversations. The research of R.D. is supported by NSF grants DMS 0612992 and Research and Training Grant DMS 0636606. The work of P.G. and M.S. is supported in part by the DOE under contract DE-FG02-95ER40893, the National Science Foundation under Grant No. PHY99-07949, the National Science and Engineering Council (NSERC) of Canada, and by a start-up grant at Bryn Mawr College. P.G. thanks ETH Zurich, the CERN TH Division, Perimeter Institute, and the University of British Columbia for hospitality. M.S. thanks the University of Toronto, the Aspen Center

for Physics, and the CERN TH divison for hospitality during the course of this work, as well as the University of Pennsylvania for continued hospitality. 


\section{A Braiding operations and monodromy matrices}

\section{A.1 Elliptic fiber}

As described at the end of Sec. 2.3, when a $(p, q) 7$-brane is transported across the branch cut of another 7 -branes, its $(p, q)$ type changes. The reason is simple: Consider a $(p, q)$ string ending on a $(p, q)$ 7-brane, and transport the 7-brane through a branch cut. From Eq. (2.3), we know that the $(p, q)$ charge of the string transforms when it crosses the branch cut. Therefore, the charge of 7-brane on which it ends must transform in the same way. We will use the symbol $\cong$ to denote equivalences under such braiding operations, i.e., under brane motions of type IIB string theory or motions of singular fibers of elliptic fibrations. The basic relation is

$$
\mathbf{X}_{\mathbf{z}_{1}} \mathbf{X}_{\mathbf{z}_{1}} \cong \mathbf{X}_{\mathbf{z}_{2}} \mathbf{X}_{\mathbf{z}_{1}^{\prime}}
$$

via motion of the brane or singular fiber $\mathbf{X}_{\mathbf{z}_{1}}$ counterclockwise through the branch cut of $\mathbf{X}_{\mathbf{z}_{2}}$. Here, $\mathbf{z}_{i}=\left(\begin{array}{c}p_{i} \\ q_{i}\end{array}\right)$, and

$$
\mathbf{z}_{1}^{\prime}=K_{\left[p_{1}, q_{1}\right]} \mathbf{z}_{1} .
$$

The transformation of the monodromy matrix follows by writing

$$
K_{\mathbf{z}_{2}} K_{\mathbf{z}_{1}}=K_{\mathbf{z}_{1}^{\prime}} K_{\mathbf{z}_{2}}
$$

from which we deduce that

$$
K_{\mathbf{z}_{1}^{\prime}}=K_{\mathbf{z}_{2}} K_{\mathbf{z}_{1}} K_{\mathbf{z}_{2}}^{-1} .
$$

The monodromy matrix of $\mathbf{X}_{\mathbf{z}_{1}}$ is conjugated by that of the branch cut it crosses.

As examples of braiding, we now explain how to realize the $S O(2 N)$ enhancement from $N<4$ D7-branes at an O7-plane, in terms of nonperturbative description of the type IIB $T^{2} / \mathbb{Z}_{2}$ orientifold, with $24(p, q) 7$-branes (F-theory on $\mathrm{K} 3$, with $24 \mathrm{I}_{1}$ fibers). It is not immediately apparent how the enhancement occurs in the nonperturbative description, since the collection $\mathbf{A}^{N} \mathbf{B C}$ cannot be coalesced for $N<4$. We provide explicit brane motions that make the $D_{1} \cong A_{1}, D_{2} \cong A_{1} \oplus A_{1}$ and $D_{3} \cong A_{3}$ lattices manifest on subcollections of $(p, q)$ 7-branes.

In addition to $\mathbf{A}, \mathbf{B}$ and $\mathbf{C}$ defined in Sec. 2.3, it is also convenient below to define $\mathbf{D}=\mathbf{X}_{0,1}$ and $\mathbf{E}=\mathbf{X}_{1,2}$, with monodromy matrices

$$
K_{\mathbf{D}}=\left(\begin{array}{ll}
1 & 0 \\
1 & 1
\end{array}\right) \quad \text { and } \quad K_{\mathbf{E}}=\left(\begin{array}{ll}
3 & -1 \\
4 & -1
\end{array}\right)
$$

respectively. Note the following useful braiding relations,

$$
\begin{aligned}
& \mathrm{DA} \cong \mathrm{AB} \cong \mathrm{BD}, \quad \mathrm{CD} \cong \mathrm{DE}, \\
& \mathrm{DC} \cong \mathrm{CA} \cong \mathrm{AD}, \quad \mathrm{BC} \cong \mathrm{CX}_{3,1},
\end{aligned}
$$


which can be combined to give

$$
\mathrm{CA}^{2} \cong \mathbf{A}^{2} \mathbf{B} \text { and } \mathbf{A B C} \cong \mathbf{B C A}
$$

Most of these relations are used below.

For $N=1$, we have

$$
\mathrm{ABC} \cong \mathrm{BCA} \cong \mathrm{BAD} \cong \mathrm{AD}^{2} \cong \mathrm{C}^{2} \mathrm{~A}
$$

Coalescing the $\mathbf{D}^{2}$ or $\mathbf{C}^{2}$ in the last two expressions gives $A_{1} \cong D_{1}$ ( $\mathrm{I}_{2}$ reducible fiber).

For $N=2$, we have

$$
\mathrm{A}^{2} \mathrm{BC} \cong \mathrm{BCA}^{2} \cong \mathrm{BA}^{2} \mathrm{~B} \cong \mathrm{B}^{2} \mathrm{D}^{2}
$$

Coalescing the $\mathbf{B}^{2}$ and $\mathbf{D}^{2}$ gives $A_{1} \oplus A_{1} \cong D_{2}\left(\mathrm{I}_{2}\right.$ fiber at two different points on $\left.\mathbb{P}^{1}\right)$.

For $N=3$, we have

$$
\begin{aligned}
\mathrm{A}^{3} \mathrm{BC} & \cong \mathrm{ABCA}^{2} \cong \mathrm{ABA}^{2} \mathrm{~B} \cong \mathrm{AB}^{2} \mathrm{D}^{2} \\
& \cong \mathrm{DABD}^{2} \cong \mathrm{DBD}^{3} \cong \mathrm{D}^{4} \mathrm{E}
\end{aligned}
$$

Coalescing the $\mathbf{D}^{4}$ gives $A_{3} \cong D_{3}$ ( $\mathrm{I}_{4}$ reducible fiber).

For $N \geq 4, \mathbf{A}^{N} \mathbf{B C}$ can be coalesced to give a $D_{N}$ singularity (I ${ }_{N-4}^{*}$ fiber) of the elliptic surface.

\section{A.2 Abelian surface fiber}

For the abelian surface fibrations $\mathcal{X}_{m, n}$ studied in this paper, we similarly define $\mathbf{X}_{[p, q], i}$ fibers by the monodromy matrices

$$
\begin{gathered}
K_{[p, q], i}=T_{i} K_{[p, q], 2} T_{i}^{-1} \text { for } i=1,3,4, \\
K_{[p, q], 2}=K_{[p, q]} \oplus I_{2 \times 2}=\left(\begin{array}{cc}
K_{p, q} & \\
& I_{2 \times 2}
\end{array}\right),
\end{gathered}
$$

where the matrices $T_{i}(m, n)$ are given in Eq. (D.11) below. The vanishing cycles of the $\mathbf{X}_{[p, q], i}$ fibers are of the form $(p, q, *, *)$. Explicitly, for $i=2$ the vanishing cycle is $(p, q, 0,0)$, while for $i \neq 2$ it is $(p, q, 0,0) T_{i}^{\mathrm{T}}$. Defining $\mathbf{A}_{i}, \mathbf{B}_{i}, \mathbf{C}_{i}, \mathbf{D}_{i}$ and $\mathbf{E}_{i}$ in this way, we find that $K_{\mathbf{A}_{i}}$ is independent of $i$, so we drop the subscript. Then, by similarity transformation (using $T_{i}$ ), the braiding relations for elliptic fibrations in the previous subsection all remain valid for abelian surface fibrations, provided that fixed $i$ is used throughout each relation.

\section{B Complex tori, abelian varieties, and the Mordell-Weil lattice}

First, note the following definitions [28, 23]: 
- Complex torus. Starting from the vector space $\mathbb{C}^{g}$, and a discrete lattice $\Lambda \subset C^{g}$ of maximal rank $2 g$, we define a complex torus by the quotient $T^{2 g}=\mathbb{C}^{g} / \Lambda$. For example, a complex $T^{2}$ is the quotient of $\mathbb{C}$ by $\Lambda=\mathbb{Z}+\tau \mathbb{Z}$.

- Group law. Addition of vectors endows $\mathbb{C}^{g}$ with the structure of an abelian group, such that $\Lambda$ is a subgroup, and $T^{2 g}$ a quotient group. The group law $T^{2 g} \times T^{2 g} \rightarrow T^{2 g}$ is just addition of points modulo $\Lambda$.

- Abelian variety. A complex torus is called an abelian variety if it admits an embedding in projective space. For a $T^{2}$, this embedding is always possible and the abelian variety is called an elliptic curve.

- Weierstrass model. An elliptic curve $E$ over the complex numbers is given by the Weierstrass model,

$$
z y^{2}=4 x^{3}-g_{2} x z^{2}-g_{3} z^{3}, \quad g_{2}, g_{3}, \in \mathbb{C},
$$

where $[x, y, z] \in \mathbb{P}^{2}$. In this case, the explicit map from points on the complex torus $t \in \mathbb{C}^{2} /(\mathbb{Z}+\tau \mathbb{Z})$ to points on the elliptic curve is $[x, y, z]=\left[\wp(t), \wp^{\prime}(t), 1\right]$, where $\wp$ is the Weierstrass $\wp$-function. The complex modulus $\tau$ is determined by the Weierstass $j$-function.

- Rational points. When the coeffients of the defining polynomials of an abelian variety $A$ over $\mathbb{C}$ are rational numbers, we can consider the subgroup $A(\mathbb{Q})$ of rational points on $A$-those points whose projective coordinates are also rational. (For example if $g_{2}, g_{3} \in \mathbb{Q}$ in the Weierstrass model, the rational points are solutions with $x, y \in \mathbb{Q}$.)

- Mordell's theorem. The group of rational points of an abelian variety is known as the Mordell-Weil group. Mordell's theorem states that this group is finitely generated:

$$
A(\mathbb{Q})=\mathbb{Z}^{\oplus r} \oplus A(\mathbb{Q})_{\text {tor }}, \quad \text { where } \quad A(\mathbb{Q})_{\text {tor }}=\oplus_{i=1}^{\operatorname{dim} A} \mathbb{Z}_{m_{i}}
$$

for positive integers $m_{i}$.

- Mordell-Weil lattice. An abelian variety $A$ over $\mathbb{C}$ together with a symmetric divisor, determines a canonical height function on the points of $A$, and a corresponding inner product. This inner product gives $A(\mathbb{Q}) / A(\mathbb{Q})_{\text {tor }}$ the structure of a lattice, known as the Mordell-Weil lattice. (For further details, including the definitions of height function and symmetric divisor, which will not concern us here, see Ref. [34].)

We can also work over a general number field $k{ }^{39}$ In this case, an abelian variety is a defined to be a projective variety - the simultaneous solution to polynomial equations in $k$-projective

\footnotetext{
${ }^{39} \mathrm{~A}$ number field is a finite extension of $\mathbb{Q}$.
} 
space - together with the additional structure of an abelian group. In the discussion of complex tori, we obtain one such interpretation for each embedding of $k$ in $\mathbb{C}$. The remaining definitions can be carried over to the more general case, and Mordell's theorem remains valid.

One reason to consider this generalization is that it allows us to consider an abelian fibration

$$
\pi: \mathcal{X} \rightarrow \mathcal{B} \text { with abelian fiber } A
$$

to be an abelian variety in its own right:

- Rational functions. Given a field $k$, let $K[x]$ denote the ring of polynomial functions of $x$ with coefficients in $k$. The field of rational functions $K(x)$ consists of functions that are ratios of polynomials in $K[x]$.

- Abelian fibration over $\mathcal{B}$. Consider an abelian variety over the function field $K=k(\mathcal{B})$ : the field of rational functions on the base $\mathcal{B}$. The defining polynomials are functions on $\mathcal{B}$. By evaluating these functions, we obtain an abelian variety over $k$ at each point of $\mathcal{B}$, i.e., an abelian fibration. A single rational point over $K$ gives a rational point of the fiber over every point of the base, i.e., a section. The Mordell-Weil group is thus the group of rational sections of the fibration modulo constant sections.

For example, in the Weierstrass model, if we take $g_{2}(w), g_{3}(w) \in \mathbb{C}(w)$ to be rational functions on $\mathbb{P}^{1}$, then the rational points are those solutions $[x(w), y(w), 1]$ such that $x$ and $y$ are also rational functions on $\mathbb{P}^{1}$. For each point $w \in \mathbb{P}^{1}$, the Weierstrass model gives a single elliptic curve over $\mathbb{C}$. Each rational point $[x(w), y(w), 1]$ gives a section: a map from $\mathbb{P}^{1}$ to the elliptic fibration. For this reason, we generally use the terminology rational section rather than rational point.

Given a complex torus $\mathbb{C}^{g} / \Lambda \cong T^{2 g}$, it is natural to ask under what conditions the torus is an abelian variety, i.e., can be embedded in complex projective space. This leads to the following definitions:

- Hodge form. A complex torus is an abelian variety if there exists a Hodge form $\omega$ : a closed positive form of type $(1,1)$ representing an integer cohomology class. (See Ref. [28], p. 302.).

- Polarization. When such a form exists, the cohomology class [ $\omega]$ is called a polarization, and can be represented by an invariant form

$$
\omega=\sum_{i=1}^{g} \delta_{i} d x^{i} \wedge d y^{i} \quad \text { with } \quad \delta_{i} \mid \delta_{i+1},
$$

in terms of coordinates $x^{i} \cong x^{i}+1$ and $y^{i} \cong y^{i}+1$ dual to a suitably chosen integer basis of the lattice $\Lambda$. The positive integers $\delta_{i}$ are called the elementary divisors of the polarization. The class $[\omega]$ is a principal polarization if $\delta_{i}=1$ for all $i$ [28]. 


\section{Mordell-Weil height pairing from intersections}

As mentioned in the previous section, the Mordell-Weil group modulo torsion $\mathrm{MW} / \mathrm{MW}_{\text {tor }}$ of an abelian fibration $\mathcal{X}$ can be given the structure of a lattice. The lattice inner product is determined by the homological intersection pairing on a surface in $\mathcal{X}$. However, this first requires a map from $\mathrm{MW}(\mathcal{X})$ to $H_{2}(\mathcal{X})$. Here, we describe the map and resulting inner product, including a slight subtlety regarding the integrality of the map when $\mathcal{X}$ has reducible fibers (related to the weakly integral junctions of Sec. 2.6).

\section{C.1 Elliptic fibration over a curve}

The theory of the Mordell-Weil lattice of an elliptic surface $\pi: \mathcal{X} \rightarrow \mathcal{B}$ was given by Shioda in Refs. [50, 51]. We follow Ref. [51] closely. Let $P$ and $Q$ denote rational sections of the elliptic fibration, $O$ the zero section, and $[P],[Q],[O]$ the corresponding divisor classes in $\operatorname{NS}(\mathcal{X})$. Let $F$ denote the class of the generic fiber. We assume that there is at least one singular fiber.

\section{Irreducible fibers}

In the case that the singular fibers are all irreducible, the inner product $\langle P, Q\rangle$ on the Mordell-Weil lattice of $\mathcal{X}$ is simply the intersection pairing

$$
\langle P, Q\rangle=[P]^{\perp} \cdot[Q]^{\perp},
$$

where $[P]^{\perp}=[P]-a[O]-b F \in H_{2}(S, \mathbb{Z})$, with $a$ and $b$ chosen so that $[P]^{\perp} \cdot[O]=[P]^{\perp} \cdot F=0$. We can compute $a$ and $b$ explicitly using

$$
\sigma^{2}=-\chi, \quad \sigma \cdot F=1, \quad \text { and } \quad F^{2}=0
$$

Here, $\sigma$ is the class of any section and $\chi$ is the arithmetic genus ${ }^{40}$ of $\mathcal{X}$. The result is $a=1$ and $b=[P] \cdot[O]+\chi$. This defines an embedding of the Mordell-Weil lattice,

$$
\varphi: \mathrm{MW} / \mathrm{MW}_{\text {tor }} \hookrightarrow H_{2}(\mathcal{X}, \mathbb{Z}), \text { such that } P \mapsto[P]^{\perp} .
$$

\section{Reducible fibers}

When the elliptic fibration has reducible fibers, a similar story holds. However, in this case, the best we can do is to embed the Mordell-Weil lattice in $H_{2}(\mathcal{X}, \mathbb{Q})$. Let $F_{p}=\pi^{-1}(p)$ denote the fiber over a point $p \in \mathcal{B}$, and

$$
R=\left\{p \in \mathcal{B} \mid F_{p} \text { is reducible }\right\} .
$$

\footnotetext{
${ }^{40}$ The arithmetic genus is defined by $\chi(\mathcal{X})=h^{2,0}-h^{1,0}+h^{0,0}$. For $\mathcal{X}$ a $\mathrm{dP}_{9}$, this gives $\chi=1$ and for a K3 surface, $\chi=2$.
} 
Then, for each $p \in \mathcal{B}$, we can decompose $F_{p}$ into irreducible components $F_{p, i}$. For simplicity, we assume that each component appears with multiplicity 1 , so that

$$
F_{p}=\sum_{i=0}^{m_{p}-1} F_{p, i} .
$$

We label the components so that $F_{p, 0}$ is the unique component that intersects the zero section. Let $T$ denote the subgroup of $\operatorname{NS}(\mathcal{X})$ generated by components of fibers and $[O]$. Under the assumption that the elliptic surface $\mathcal{X}$ has at least one singular fiber, both $\operatorname{NS}(\mathcal{X})$ and $T$ are torsion-free. The Mordell-Weil group is the quotient

$$
\operatorname{MW}(\mathcal{X})=\mathrm{NS}(\mathcal{X}) / T .
$$

To define the Mordell-Weil lattice, we seek an embedding $\varphi: \mathrm{MW} / \mathrm{MW}_{\text {tor }} \hookrightarrow H_{2}(\mathcal{X}, \mathbb{Q})$. We can proceed as before, this time requiring that $[P]^{\perp}$ in the inner product (C.1) lie in the orthogonal complement of $[O], F$, and all components $F_{p, i}$ of reducible fibers. The analog of Eq. (C.3) is

$$
\varphi: \mathrm{MW} / \mathrm{MW}_{\text {tor }} \hookrightarrow H_{2}(\mathcal{X}, \mathbb{Q}), \quad \text { taking } \quad P \mapsto[P]^{\perp},
$$

where

$$
[P]^{\perp}=[P]-[O]-([P] \cdot[O]+\chi) F-\sum_{p \in R}\left(F_{p, 1}, \ldots, F_{p, m_{p-1}}\right) A_{p}^{-1}\left(\begin{array}{c}
{[P] \cdot F_{p, 1}} \\
\vdots \\
{[P] \cdot F_{p, m_{p-1}}}
\end{array}\right) .
$$

Here, $A_{p}$ is the intersection matrix of the extra $(i \neq 0)$ components of the reducible fiber $F_{p}$,

$$
A_{p}=F_{p, i} \cdot F_{p, j}, \quad \text { where } 1 \leq i, j \leq m_{p}-1 .
$$

Since $A_{p}^{-1}$ is not integral, $[P]^{\perp}$ lies in $\operatorname{NS}(\mathcal{X})_{\mathbb{Q}}=\operatorname{NS}(\mathcal{X}) \otimes \mathbb{Q}$ but not in $\operatorname{NS}(\mathcal{X})$.

In fact, we can be more explicit about how close to integrality $\operatorname{Im} \varphi$ is. Define the essential sublattice of $\mathrm{NS}(\mathcal{X})$ to be $L=T^{\perp}$, and let $\ell=\operatorname{lcm}\left\{m_{p} \mid p \in R\right\}$. Then,

$$
\operatorname{Im} \varphi \subset \frac{1}{\ell} L .
$$

This is the geometric analog of the weak integrality condition on string junctions in Sec. 2.6.

Shioda goes on to show that the narrow Mordell-Weil lattice is

$$
\mathrm{MW}_{0} \cong L,
$$

and that

$$
\mathrm{MW} / \mathrm{MW}_{\text {tor }} \subset L^{*}
$$

where $L^{*}$ is the lattice dual to $L$ :

$$
L^{*}=\{x \in L \otimes \mathbb{Q} \mid\langle x, y\rangle \in \mathbb{Z} \text { for all } y \in L\} .
$$




\section{The unimodular case}

When $N S(\mathcal{X})$ is unimodular, Eq. (C.12) becomes an equality. This is the case, for example, for $\mathcal{X}=\mathrm{dP}_{9}$. Using this result, Shioda also gives a useful expression for the torsion subgroup of the Mordell-Weil group. It is

$$
\mathrm{MW}_{\text {tor }} \cong T / T^{\prime}
$$

where $T^{\prime}=(T \otimes \mathbb{Q}) \cap \mathrm{NS}(\mathcal{X})$.

\section{C.2 Abelian surface fibration over a curve}

The description of the Mordell-Weil lattice of an abelian surface fibration, or higher dimensional abelian fibration, is very similar to that for an elliptic fibration. Here, we sketch the new ingredients necessary to define the height pairing in the general case.

On a smooth compact $n$-dimensional complex manifold $\mathcal{X}$, any class $c \in H^{2}(\mathcal{X}, \mathbb{R})$ gives a map

$$
H^{a}(\mathcal{X}, \mathbb{R}) \rightarrow H^{2 n-a}(\mathcal{X}, \mathbb{R}),
$$

given by cup product with the $(n-a)$ th power of $c$. When $c$ is a Kähler class, and in particular when $c$ is the Chern class $c_{1}(L)$ of an ample line bundle $L$ on a smooth projective variety, the Hard Lefschetz theorem (cf. Ref. [28], p. 122) says that this map is an isomorphism for $a$ between 0 and $n$. (The real coefficients can be replaced throughout by the rationals, but the result fails over the integers.) We apply this in our case, with $n=3, a=2$, where $L$ is an ample theta divisor. This allows us to identify $H_{2}(X)=H^{4}(X)$ with $H^{2}(X)$. The natural height pairing on the Mordell-Weil lattice is determined in terms of this identification.

\section{Monodromy matrices for the abelian fibration $\mathcal{X}_{m, n}$}

First let us fix conventions in the simpler elliptically fibered case. In Sec. 2.3, the monodromy matrices $K$ were defined to act on vectors $\left(\begin{array}{l}p \\ q\end{array}\right)$, that is, on the components of the homology class $\mathbf{z}=p \alpha+q \beta$ relative to a basis $\{\alpha, \beta\}$ of $H_{1}\left(T^{2}, \mathbb{Z}\right)$. Let $y^{i} \cong y^{i}+1$ denote coordinates on the $T^{2}$. Then, a convenient basis for $H^{1}\left(T^{2}, \mathbb{Z}\right)$ is $\left\{\left[d y^{i}\right]\right\}$, and a corresponding basis for $H_{1}\left(T^{2}, \mathbb{Z}\right)$ is $\alpha=\left[S_{1}^{1}\right], \beta=\left[S_{2}^{1}\right]$, where the circles $S_{i}^{1}$ are chosen so that

$$
\int_{S_{i}^{1}} d y^{i}=\delta_{j}^{i} .
$$

In this basis, a class $[\omega] \in H^{1}\left(T^{2}, \mathbb{Z}\right)$ can be represented by $\omega=\omega_{i} d y^{i}$ and a class $[\mathbf{z}] \in$ $H_{1}\left(T^{2}, \mathbb{Z}\right)$ by $\mathbf{z}=z^{i} S_{i}^{1}$. Then, $\left(\begin{array}{l}z^{1} \\ z^{2}\end{array}\right)=\left(\begin{array}{l}p \\ q\end{array}\right)$, and by a slight abuse of notation we simply write $\mathbf{z}=\left(\begin{array}{l}p \\ q\end{array}\right)$ as in Sec. 2.3. 
When a branch cut is crossed in the positive (counterclockwise) direction about the branch point, the components and basis elements transform as

$$
\begin{aligned}
z^{i} & \mapsto K^{i}{ }_{j} z^{j}, & S_{i}^{1} \mapsto S_{j}^{1}\left(K^{-1}\right)^{j}{ }_{i}, \\
\omega_{i} & \mapsto \omega_{j}\left(K^{-1}\right)^{j}{ }_{i}, & d y^{i} \mapsto K^{i}{ }_{j} d y^{j} .
\end{aligned}
$$

These definitions all carry over to the case of $T^{4}$ fiber except that $i=1,2,3,4$ and we write $\mathbf{z}^{T}=(p, q, r, s)$, by the same slight abuse of notation.

Warm-up: $\mathcal{N}=4$ case, $\mathrm{K} 3 \times T^{2}$

Before describing the monodromy matrices for the abelian surface fibration $\mathcal{X}_{m, n}$, it is useful to consider the $\mathcal{N}=4$ case. In the absence of flux, the IIA dual of the type IIB $T^{6} / \mathbb{Z}_{2}$ orientifold is a compactification on $\mathrm{K} 3 \times T^{2}$, which we can think of as a $T^{4}$ fibration over $\mathbb{P}^{1}$ in which a $T^{2} \subset T^{4}$ trivially factorizes. Let $y^{1}, y^{2}$ denote the coordinates on the nontrivial $T^{2}$ fiber of K3, $y^{3}, y^{4}$ coordinates on the trivial $T^{2}$, and $y^{5}, y^{6}$ coordinates on the $\mathbb{P}^{1}$ base. ${ }^{41}$ The monodromy matrices for K3 were given in Sec. 2.3. The collection of singular fibers is $\mathbf{A}^{16} \mathbf{B C B C B C B C}$, with matrices $K_{\mathbf{A}}, K_{\mathbf{B}}$ and $K_{\mathbf{C}}$ given by Eq. (2.4). To obtain the corresponding monodromy matrices on the $T^{4}$ fibration $\mathrm{K} 3 \times T^{2}$, we simply tensor with the identity matrix in the $y^{3} y^{4}$ block:

$$
K_{\mathbf{A}}=\left(\begin{array}{cccc}
1 & -1 & 0 & 0 \\
0 & 1 & 0 & 0 \\
0 & 0 & 1 & 0 \\
0 & 0 & 0 & 1
\end{array}\right), \quad K_{\mathbf{C}}=\left(\begin{array}{cccc}
2 & -1 & 0 & 0 \\
1 & 0 & 0 & 0 \\
0 & 0 & 1 & 0 \\
0 & 0 & 0 & 1
\end{array}\right), \quad K_{\mathbf{B}}=\left(\begin{array}{cccc}
0 & -1 & 0 & 0 \\
1 & 2 & 0 & 0 \\
0 & 0 & 1 & 0 \\
0 & 0 & 0 & 1
\end{array}\right)
$$

In the classical supergravity duality (cf. end of Sec. 3.1), only the combined monodromy of the pair $\mathbf{O}=\mathbf{B C}$ is visible, with monodromy

$$
K_{\mathbf{O}}=K_{\mathbf{C}} K_{\mathbf{B}}=\left(\begin{array}{cccc}
-1 & 4 & 0 & 0 \\
0 & -1 & 0 & 0 \\
0 & 0 & 1 & 0 \\
0 & 0 & 0 & 1
\end{array}\right)
$$

The fact that $K_{\mathbf{A}}$ and $K_{\mathbf{O}}$ have zeros in the 2,3,4 components of the first column reflects the duality origin of $\mathrm{K} 3 \times T^{2}$ in the M-theory lift from the $T^{3} / \mathbb{Z}_{2} \times T^{3}$ orientifold: The $y^{1}$ direction is the M-theory circle, and in the perturbative lift, this circle is fibered over the other directions.

\footnotetext{
${ }^{41}$ Compared to Ref. [46], we have $\left(y^{1}, y^{2}, y^{3}, y^{4}, y^{5}, y^{6}\right)$ here $=\left(x^{10},-x^{8}, x^{4}, x^{5}, x^{6}, x^{7}\right)$ there
} 
$\mathcal{N}=2$ case, $\mathcal{X}_{m, n}$

In the $\mathcal{N}=2$ case, the manifold $\mathcal{X}_{m, n}$ arises as follows. We first T-dualize the IIB $T^{6} / \mathbb{Z}_{2}$ orientifold along a $T^{3}$ to obtain a IIA D6/O6 orientifold. The orientifold is not quite $T^{3} / \mathbb{Z}_{2} \times$ $T^{3}$, since the IIB NS flux dualizes to twists of the $T^{3} \times T^{3}$ topology. Instead, the orientifold is $\mathcal{Y}_{n}\left(y^{2}, y^{3}, y^{4}, y^{5}, y^{6}\right) / \mathbb{Z}_{2} \times S^{1}$, where $\mathcal{Y}_{n}$ is an $S_{3}^{1} \times S_{4}^{1}$ fibration over $T_{\{2,5,6\}}^{3}$. The global 1-forms on $\mathcal{Y}_{n}$ are $d y^{2}, \eta^{3}, \eta^{4}, d y^{5}$, and $d y^{6}$, with

$$
d \eta^{3}=2 n d y^{2} \wedge d y^{5}, \quad d \eta^{4}=2 n d y^{2} \wedge d y^{6} .
$$

Up to a choice of coordinate gauge (equivalent to a gauge choice for the NS $B$-field in $T^{6} / \mathbb{Z}_{2}$ ), we can take

$$
\eta^{3}=d y^{3}+2 n y^{2} d y^{5}, \quad \eta^{4}=d y^{4}+2 n d y^{2} .
$$

The $\mathbb{Z}_{2}$ involution is $(-1)^{F_{L}} \Omega \mathcal{I}_{3}$, where $F_{L}$ is left moving fermion number, $\Omega$ is worldsheet parity, and $\mathcal{I}_{3}$ is the inversion $\mathcal{I}_{3}:\left(y^{2}, y^{5}, y^{6}\right) \mapsto-\left(y^{2}, y^{5}, y^{6}\right)$, which acts on the 1 -forms as

$$
\mathcal{I}_{3}^{*}:\left(d y^{2}, \eta^{3}, \eta^{4}, d y^{5}, d y^{6}\right) \mapsto\left(-d y^{2}, \eta^{3}, \eta^{4},-d y^{5},-d y^{6}\right) .
$$

This type IIA orientifold lifts to M-theory on $\mathcal{X}_{m, n} \times S^{1}$. Compactifying on the $S^{1}$ factor then gives the type IIA Calabi-Yau compactification on $\mathcal{X}_{m, n}$. In the classical supergravity description of the lift, $\mathcal{X}_{m, n}$ is obtained by fibering the M-theory circle over $\mathcal{Y}_{m, n}$ and then quotienting by the $\mathbb{Z}_{2}$ involution $\mathcal{I}_{4}:\left(\eta^{1}, y^{2}, y^{5}, y^{6}\right) \mapsto-\left(\eta^{1}, y^{2}, y^{5}, y^{6}\right)$. Here, $\eta^{1}$ is the 1 -form along the M-theory circle, and satisfies

$$
\begin{aligned}
d \eta^{1} & =F_{(2)}^{\text {IIA orientifold }} /\left(2 \pi \sqrt{\alpha^{\prime}}\right) \\
& =-2 m \eta^{3} \wedge d y^{6}+2 m \eta^{4} \wedge d y^{5}+(\text { warp factor dependence })
\end{aligned}
$$

This leading order description falls short of the exact description of $\mathcal{X}_{m, n}$, since it ignores KK modes around the M-theory circle, and breaks the $U(1)$ isometry only by the explicit $\mathbb{Z}_{2}$. Nevertheless, it contains sufficient information to parametrize the exact description.

In summary, at the level of this description, the steps to construct $\mathcal{X}_{m, n}$ are

1. Fiber the $S_{3}^{1}$ and $S_{4}^{1}$ circles over $T_{\{2,5,6\}}^{3}$,

2. Fiber the $S_{1}^{1}$ circle over the resulting manifold $\mathcal{Y}_{n}$,

3. Quotient by $\mathcal{I}_{4}$, which inverts the $1,2,4,5$ directions.

To make the abelian surface fibration manifest, step 1 can be equivalently described as $1^{\prime}$. Fiber the torus $T_{\{2,3,4\}}^{3}$ over $T_{\{5,6\}}^{2}$, 
Then, the abelian surface fibration $\mathcal{X}_{m, n}$ can be understood as the $T_{\{1,2,3,4\}}^{4}$ fibration over $T_{\{5,6\}}^{2}$, quotiented by $\mathcal{I}_{4}$. The base of the resulting $T^{4}$ fibration is $T_{\{5,6\}}^{2} / \mathbb{Z}_{2} \cong \mathbb{P}^{1}$.

The collection of singular fibers visible in this description is $\mathrm{A}^{M} \mathbf{O}_{1} \mathbf{O}_{2} \mathbf{O}_{3} \mathbf{O}_{4}$, where the the locations of the $\mathbf{O}_{i}$ on $\mathbb{P}^{1} \cong T_{\{5,6\}}^{2} / \mathbb{Z}_{2}$ are the $\mathbb{Z}_{2}$ fixed points $p_{i}$, with $\left(y^{5}, y^{6}\right)$ coordinates

$$
p_{1}=(1 / 2,0), \quad p_{2}=(0,0), \quad p_{3}=(0,1 / 2), \quad p_{4}=(1 / 2,1 / 2) .
$$

With the appropriate coordinate gauge choice for $y^{1}$, the monodromies $K_{\mathbf{A}}$ and $K_{\mathbf{O}_{i}}$ are the same as those for $\mathrm{K} 3 \times T^{2}$. That is, $K_{\mathbf{O}_{2}}=K_{\mathbf{O}}$ of Eq. (D.4). However, the remaining monodromies $K_{\mathbf{O}_{i}}$ differ from $K_{\mathbf{O}}$. We deduce these monodromies as follows.

In the basis $\eta^{1}, d y^{2}, \eta^{3}, \eta^{4}$ (restricted to the $T^{4}$ fiber), the monodromies $K_{\mathbf{O}_{i}}$ at all four fixed points on the base are simply

$$
\left(\begin{array}{cccc}
-1 & 0 & 0 & 0 \\
0 & -1 & 0 & 0 \\
0 & 0 & 1 & 0 \\
0 & 0 & 0 & 1
\end{array}\right)
$$

from the $\mathbb{Z}_{2}$ action on the fiber. However, we seek the monodromies in the coordinate basis $d y^{1}, d y^{2}, d y^{3}, d y^{4}$ instead. ${ }^{42}$ Since the fixed points $p_{i}$ are locally equivalent to one another, the monodromy matrices $K_{\mathbf{O}_{i}}$ must be related by similarity transformation,

$$
K_{\mathbf{O}_{i}}=T_{i} K_{\mathbf{O}_{2}} T_{i}^{-1}, \quad \text { where } \quad S_{i} \in \mathrm{SL}(4, \mathbb{Z})
$$

To deduce the transformation matrices $T_{i}$ we first use the definition (D.6) to determine the lower $3 \times 3$ block. From

$$
\begin{array}{ll}
\left.\eta^{3}\right|_{y^{5}=\frac{1}{2}}=d y^{3}-n d y^{2}, & \left.\eta^{3}\right|_{y^{5}=0}=d y^{3}, \\
\left.\eta^{4}\right|_{y^{6}=\frac{1}{2}}=d y^{4}-n d y^{2}, & \left.\eta^{4}\right|_{y^{6}=0}=d y^{4},
\end{array}
$$

we obtain the lower $3 \times 3$ blocks of the following matrices:

$$
T_{1}=\left(\begin{array}{cccc}
1 & 0 & 0 & -m \\
0 & 1 & 0 & 0 \\
0 & -n & 1 & 0 \\
0 & 0 & 0 & 1
\end{array}\right), \quad T_{3}=\left(\begin{array}{cccc}
1 & 0 & m & 0 \\
0 & 1 & 0 & 0 \\
0 & 0 & 1 & 0 \\
0 & -n & 0 & 1
\end{array}\right), \quad T_{4}=\left(\begin{array}{cccc}
1 & 0 & m & -m \\
0 & 1 & 0 & 0 \\
0 & -n & 1 & 0 \\
0 & -n & 0 & 1
\end{array}\right)
$$

with $T_{2}$ equal to the $4 \times 4$ identity. The zeros in the first column follow from the fact that the M-theory circle is fibered over $\mathcal{Y}_{n}$ in our construction. The first row can be determined by a

\footnotetext{
${ }^{42}$ Recall that the homology components $z^{i}=(p, q, r, s)$ and cohomology basis transform in the same way under monodromy transformations (cf. Eq. (D.2)).
} 
careful analysis of the connection for the M-theory circle fibration, however, a simpler route is to note that the similarity transformations $T_{i}$ must leave the Hodge form (3.6) invariant. This determines the first row except for the second component. Finally, this component is required to vanish so that the $T_{i}^{-1}$ are also integral and $T_{i} \in S L(4, \mathbb{Z})$.

Eqs. (D.4) and (D.12) together give

$$
\begin{aligned}
K_{\mathbf{O}_{1}} & =\left(\begin{array}{cccc}
-1 & -4 & 0 & -2 m \\
0 & -1 & 0 & 0 \\
0 & 2 n & 1 & 0 \\
0 & 0 & 0 & 1
\end{array}\right), & & K_{\mathbf{O}_{2}}=\left(\begin{array}{cccc}
-1 & -4 & 0 & 0 \\
0 & -1 & 0 & 0 \\
0 & 0 & 1 & 0 \\
0 & 0 & 0 & 1
\end{array}\right) \\
K_{\mathbf{O}_{3}} & =\left(\begin{array}{cccc}
-1 & -4 & 2 m & 0 \\
0 & -1 & 0 & 0 \\
0 & 0 & 1 & 0 \\
0 & 2 n & 0 & 1
\end{array}\right), & & K_{\mathbf{O}_{4}}=\left(\begin{array}{cccc}
-1 & -4 & 2 m & -2 m \\
0 & -1 & 0 & 0 \\
0 & 2 n & 1 & 0 \\
0 & 2 n & 0 & 1
\end{array}\right) .
\end{aligned}
$$

While the classical supergravity duality does not resolve $\mathbf{O}_{i}=\mathbf{B}_{i} \mathbf{C}_{i}$ into its two constituents, we know that $K_{\mathbf{O}_{2}}=K_{\mathbf{O}}$ of Eq. (D.4) can be factored into the two monodromies $K_{\mathbf{B}}$ and $K_{\mathbf{C}}$ of Eq. (D.3), each related to $K_{\mathbf{A}}$ via similarity transformation. Consequently, the $K_{\mathbf{O}_{i}}$ factorize as

$$
K_{\mathbf{O}_{i}}=K_{\mathbf{C}_{i}} K_{\mathbf{B}_{i}}, \quad \text { where } \quad K_{\mathbf{B}_{i}}=T_{i} K_{\mathbf{B}} T_{i}^{-1} \quad \text { and } \quad K_{\mathbf{C}_{i}}=T_{i} K_{\mathbf{C}} T_{i}^{-1} .
$$

This gives the matrices quoted in Sec. 3.3. The factorization is unique up to braiding and overall $S L(4, \mathbb{Z})$ conjugation.

\section{E Null loop junctions of $\mathcal{X}_{m, n}$}

To determine the junction lattice vectors (3.27) of the null loop junctions of $\mathcal{X}_{m, n}$, we first transform the loop junctions to standard presentation, and then read off the number of strings emanating from each $\mathbf{A}, \mathbf{B}_{i}$ and $\mathbf{C}_{i}$ point on $\mathbb{P}^{1}$. To transform to standard presentation, we push the lower half of the loop through each branch point in succession, from left to right, applying the Hanany-Witten effect at each step (cf. Fig. 6). Once this has been done, the original loop is contractible to a point, leaving just the new Hanany-Witten strings intersecting at this point. The discontinuity in the $(p, q, r, s)$ charge of the original segment of string across a branch cut of $\mathbf{X}_{i}$ is equal to the charge $\mathbf{z}_{i}$ of the new string grown via the Hanany-Witten effect.

Consider the $(p, q, r, s)=(1,0,0,0)$ loop. After crossing each successive branch cut in the counterclockwise direction, the new $(p, q, r, s)$ charge is determined by multiplication by 


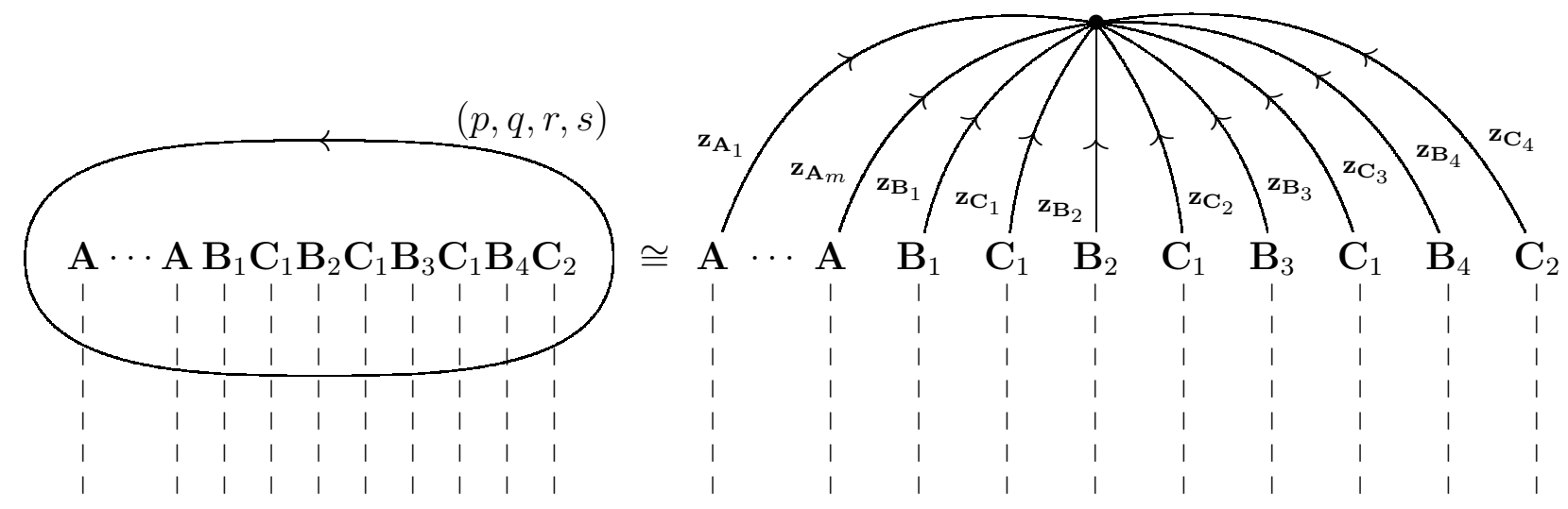

Figure 17: A loop junction of $\mathcal{X}_{m, n}$ transformed to standard presentation.

the monodromy matrices (3.13) and (3.14). For the loop on the LHS of Fig. 17, this gives

A : no change,

$\mathbf{B}_{1}:\left(\begin{array}{cccc}0 & -1 & 0 & -m \\ 1 & 2 & 0 & m \\ -n & -n & 1 & -m n \\ 0 & 0 & 0 & 1\end{array}\right)\left(\begin{array}{l}1 \\ 0 \\ 0 \\ 0\end{array}\right)=\left(\begin{array}{c}0 \\ 1 \\ -n \\ 0\end{array}\right), \quad \mathbf{B}_{3}:\left(\begin{array}{cccc}0 & -1 & m & 0 \\ 1 & 2 & -m & 0 \\ 0 & 0 & 1 & 0 \\ -n & -n & m n & 1\end{array}\right)\left(\begin{array}{l}1 \\ 0 \\ 0 \\ 0\end{array}\right)=\left(\begin{array}{c}0 \\ 1 \\ 0 \\ -n\end{array}\right)$,

$\mathbf{C}_{1}:\left(\begin{array}{cccc}2 & -1 & 0 & m \\ 1 & 0 & 0 & m \\ -n & n & 1 & -m n \\ 0 & 0 & 0 & 1\end{array}\right)\left(\begin{array}{c}0 \\ 1 \\ -n \\ 0\end{array}\right)=\left(\begin{array}{c}-1 \\ 0 \\ 0 \\ 0\end{array}\right), \quad \mathbf{C}_{3}:\left(\begin{array}{cccc}2 & -1 & -m & 0 \\ 1 & 0 & -m & 0 \\ 0 & 0 & 1 & 0 \\ -n & n & m n & 1\end{array}\right)\left(\begin{array}{c}0 \\ 1 \\ 0 \\ -n\end{array}\right)=\left(\begin{array}{c}-1 \\ 0 \\ 0 \\ 0\end{array}\right)$,

$\mathbf{B}_{2}:\left(\begin{array}{cccc}0 & -1 & 0 & 0 \\ 1 & 2 & 0 & 0 \\ 0 & 0 & 1 & 0 \\ 0 & 0 & 0 & 1\end{array}\right)\left(\begin{array}{c}-1 \\ 0 \\ 0 \\ 0\end{array}\right)=\left(\begin{array}{c}0 \\ -1 \\ 0 \\ 0\end{array}\right), \quad \mathbf{B}_{4}:\left(\begin{array}{cccc}0 & -1 & m & -m \\ 1 & 2 & -m & m \\ -n & -n & 1+m n & -m n \\ -n & -n & m n & 1-m n\end{array}\right)\left(\begin{array}{c}-1 \\ 0 \\ 0 \\ 0\end{array}\right)=\left(\begin{array}{c}0 \\ -1 \\ n \\ n\end{array}\right)$,

$\mathbf{C}_{2}:\left(\begin{array}{cccc}2 & -1 & 0 & 0 \\ 1 & 0 & 0 & 0 \\ 0 & 0 & 1 & 0 \\ 0 & 0 & 0 & 1\end{array}\right)\left(\begin{array}{c}0 \\ -1 \\ 0 \\ 0\end{array}\right)=\left(\begin{array}{l}1 \\ 0 \\ 0 \\ 0\end{array}\right), \quad \mathbf{C}_{4}:\left(\begin{array}{cccc}2 & -1 & -m & m \\ 1 & 0 & -m & m \\ -n & n & 1+m n & -m n \\ -n & n & m n & 1-m n\end{array}\right)\left(\begin{array}{c}0 \\ -1 \\ n \\ n\end{array}\right)=\left(\begin{array}{l}1 \\ 0 \\ 0 \\ 0\end{array}\right)$.

The charges $\mathbf{z}_{\mathbf{X}_{i}}$ in the tree junction on the RHS of Fig. 17, are the differences between 
succesive $(p, q, r, s)$ charges in Eq. (E.1):

$$
\begin{aligned}
& \mathbf{z}_{\mathbf{A}_{i}}=0, \quad \mathbf{z}_{\mathbf{B}_{1}}=-\left(\begin{array}{c}
1 \\
-1 \\
n \\
0
\end{array}\right), \quad \mathbf{z}_{\mathbf{C}_{1}}=-\left(\begin{array}{c}
1 \\
1 \\
-n \\
0
\end{array}\right), \quad \mathbf{z}_{\mathbf{B}_{2}}=\left(\begin{array}{c}
1 \\
-1 \\
0 \\
0
\end{array}\right), \quad \mathbf{z}_{\mathbf{C}_{2}}=\left(\begin{array}{l}
1 \\
1 \\
0 \\
0
\end{array}\right), \\
& \mathbf{z}_{\mathbf{B}_{3}}=-\left(\begin{array}{c}
1 \\
-1 \\
0 \\
n
\end{array}\right), \quad \mathbf{z}_{\mathbf{C}_{3}}=-\left(\begin{array}{c}
1 \\
1 \\
0 \\
-n
\end{array}\right), \quad \mathbf{z}_{\mathbf{B}_{4}}=\left(\begin{array}{c}
1 \\
-1 \\
n \\
n
\end{array}\right), \quad \mathbf{z}_{\mathbf{C}_{4}}=\left(\begin{array}{c}
1 \\
1 \\
-n \\
-n
\end{array}\right) .
\end{aligned}
$$

Note that $\mathbf{z}_{\mathbf{X}_{i}} \propto(p, q, r, s) \mathbf{X}_{i}$, where $(p, q, r, s) \mathbf{X}_{i}$ is the vanishing 1-cycle of $\mathbf{X}_{i}$. The factors of proportionality give the the components of the junction lattice vector. Reading off the coefficients from Eq. (E.2), we see that the lattice vector of the $(1,0,0,0)$ loop is

$$
\boldsymbol{\delta}_{1}=\left(0^{M} ;-1,-1 ; 1,1 ;-1,-1 ; 1,1\right),
$$

as claimed in Sec. 3.4. In the same way, the $(0,1,0,0),(0,0,1,0)$ and $(0,0,0,1)$ loops of $\mathcal{X}_{m, n}$ give junction lattice vectors $\boldsymbol{\delta}_{2}, m \boldsymbol{\delta}_{3}$ and $m \boldsymbol{\delta}_{4}$, respectively, where the $\boldsymbol{\delta}_{i}$ are defined in Eq. (3.23).

\section{F Complex curves and their Jacobians}

In this Appendix, we provide background on complex curves and their Jacobian varieties, and on the relation of the Jacobian variety to divisors, line bundles, and the Picard group. This review largely follows Ref. [28]. The broad picture to keep in mind is that in algebraic geometry, the Jacobian $\mathcal{J}_{C}$ of a genus- $g$ curve $C$ is a $T^{2 g}$ analogous to what a physicist would call the moduli space of $U(1)$ Wilson lines on $C$.

A genus- $g$ curve has a fundamental group $\pi_{1}(C)$ with $2 g$ generators $a_{i}$ and $b_{i}$, for $i=$ $1, \ldots g$, subject to the relation

$$
\left(a_{1} b_{1} a_{1}^{-1} b_{1}^{-1}\right)\left(a_{2} b_{2} a_{2}^{-1} b_{2}^{-1}\right) \cdots\left(a_{g} b_{g} a_{g}^{-1} b_{g}^{-1}\right)=1 .
$$

The homology group $H_{1}(C, \mathbb{Z})$ is the abelianization of $\pi_{1}(C)$, that is, $\pi_{1}(C)$ modulo its commutator subgroup $\left\{h_{1} h_{2} h_{1}^{-1} h_{2}{ }^{-1} \mid h_{1}, h_{2} \in \pi_{1}(C)\right\}$. Thus,

$$
H_{1}(C, \mathbb{Z})=\mathbb{Z}^{2 g},
$$

generated by $2 g$ linearly independent 1 -cycles $\gamma_{i}$. In a canonical basis of $A$-cycles and $B$ cycles, we choose

$$
\gamma_{i}=A_{i} \quad \text { and } \quad \gamma_{g+i}=B_{i} \quad \text { for } \quad i=1, \ldots, g,
$$


with

$$
A_{i} \cap B_{j}=\delta_{i j}, \quad A_{i} \cap A_{j}=B_{i} \cap B_{j}=0 .
$$

So far, we have taken a topological perspective. However, it is also natural to take a holomorphic perspective. The cohomology group

$$
H^{0}\left(C, \Omega^{1}\right)=\mathbb{C}^{g}
$$

is generated by $g$ holomorphic 1 -forms $\omega_{1}, \ldots, \omega_{g}$. By integration, we then have $2 g$ period vectors

$$
\Pi_{i}=\left(\begin{array}{c}
\int_{\gamma_{i}} \omega_{1} \\
\int_{\gamma_{i}} \omega_{2} \\
\vdots \\
\int_{\gamma_{i}} \omega_{g}
\end{array}\right), \quad \text { for } \quad i=1, \ldots, 2 g
$$

and a $g \times 2 g$ period matrix $\Pi=\left(\Pi_{1} \Pi_{2} \cdots \Pi_{2 g}\right)$. By $S L(3, \mathbb{C})_{L} \times S L(6, \mathbb{Z})_{R}$ change of basis, the period matrix can be put in standard form $\Pi=(I \mid \tau)$, where $I$ is the $g \times g$ identity matrix and $\tau$ is a symmetric $g \times g$ matrix.

The Jacobian $\mathcal{J}_{C}$ of a genus- $g$ curve $C$ is the complex torus

$$
\mathbb{C}^{g} / \Lambda \cong T^{2 g}
$$

where $\Lambda$ is the lattice generated by the period vectors $\Pi_{i} \in \mathbb{C}^{g}$. It is a principally polarized abelian variety, as defined in App. B, and conversely, any smooth principally polarized abelian variety is the Jacobian of some complex curve [28]. If $x^{i}$ and $y^{i}$ denote the coordinates on $\mathbb{C}^{g}$ relative to the lattice basis, then the holomorphic 1 -forms are the familiar $d x+\tau d y$ linear combinations

$$
\omega_{i}=\delta_{i j} d x^{j}+\tau_{i j} d y^{j}
$$

For a generic complex torus, $\tau$ need not have any special symmetry properties, however the existence of a polarization implies that $\tau$ is symmetric.

Given a base point $p_{0} \in C$, the Abel-Jacobi map takes points on $C$ to points on its Jacobian variety $\mathcal{J}_{C}$ :

$$
\mu: C \rightarrow \mathcal{J}_{C}, \quad \mu(p)=\left(\begin{array}{c}
\int_{p_{0}}^{p} \omega_{1} \\
\int_{p_{0}}^{p} \omega_{2} \\
\vdots \\
\int_{p_{0}}^{p} \omega_{g}
\end{array}\right) \quad \bmod \Lambda
$$

To connect this discussion to that in Sec. 4.3.3 and App. J, we now reinterpret the Abel-Jacobi map in terms of line bundles on $C$. The Picard group $\operatorname{Pic}(C)$ is the space of 
holomorphic line bundles on $C{ }^{43}$ To each divisor $D$ of $C$ (i.e., to each linear combination of points of $C$ ), is associated a line bundle $[D]$, and to each line bundle $L$ is associated its first Chern class $c_{1}(L)$. If we write $D=\sum a_{i} p_{i}$ and $L=[D]$, then $c_{1}(L)$ counts the net number of points of $D$, called the degree of $L$ :

$$
\operatorname{deg} L=\int_{C} c_{1}(L)=\sum a_{i} .
$$

We use a superscript $\operatorname{Pic}^{d}(C) \subset \operatorname{Pic}(C)$ to denote the subspace of line bundles of degree $d$. Note that the degree of a line bundle is multiplicative, $\operatorname{Pic}^{m}(C) \times \operatorname{Pic}^{n}(C) \rightarrow \operatorname{Pic}^{m+n}(C)$, so that only $\operatorname{Pic}^{0}(C)$ has a canonical group structure. In terms of $U(1)$ Wilson lines, $\operatorname{Pic}^{0}(C)$ is the space of flat connections on $C$, that is, of holomorphic potentials $A$ such that the field strength $F_{i \bar{\jmath}}$ vanishes. This makes it clear that for curves of nonzero genus, a line bundle is not uniquely determined by its first Chern class. Finally, it can be shown that each divisor $D=\sum a_{i} p_{i}$ defines a meromorphic section $(D)$ of the line bundle $[D]=\otimes\left[p_{i}\right]_{i}^{a_{i}}$, with a zero of degree $a_{i}$ at $p_{i}$ for each $a_{i}>0$ and a pole of degree $-a_{i}$ at $p_{i}$ for each $a_{i}<0[28]{ }^{44}$

In this description, the Jacobian variety of $C$ is defined to be the space of degree zero line bundles $\mathcal{J}_{C}=\operatorname{Pic}^{0}(C)$. Note that each $\operatorname{Pic}^{d}(C)$ is noncanonically isomorphic to $\operatorname{Pic}^{0}(C)$, so it is also a $T^{2 g}$. For example,

$$
\operatorname{Pic}^{1}(C) \stackrel{\cong}{\rightarrow} \operatorname{Pic}^{0}(C),
$$

by tensoring with a line bundle of degree -1 , and similarly, $\operatorname{Pic}^{d}(C) \cong \operatorname{Pic}^{0}(C)$ by tensoring with the $d$ th power of this line bundle.

The Abel-Jacobi map directly follows. A point (divisor) $p_{0}$ defines a map (F.11) via tensoring with the line bundle $\left[p_{0}\right]^{-1}=\left[-p_{0}\right]$ of degree -1 . Since any point $p \in C$ defines an element $[p] \in \operatorname{Pic}^{1}(C)$, we obtain a map from $C$ to $\mathcal{J}_{C}=\operatorname{Pic}^{0}(C)$ :

$$
\mu(p)=\left[p-p_{0}\right] .
$$

\section{G Genera of curves in $\mathbb{P}^{1} \times \mathbb{P}^{1}$}

Consider a degree $(\alpha, \beta)$ curve $B_{\alpha, \beta} \subset \mathbb{P}^{1} \times \mathbb{P}^{1}$. Let $x, y$ denote the hyperplane classes of the respective $\mathbb{P}^{1}$ factors, with $x^{2}=y^{2}=0$. By the adjunction formula,

$$
\begin{aligned}
c\left(T_{B_{\alpha, \beta}}\right) & =c\left(\mathbb{P}^{1} \times \mathbb{P}^{1}\right) / c\left(N_{B_{\alpha, \beta}}\right) \\
& =(1+x)^{2}(1+y)^{2} /(1+\alpha x+\beta y) \\
& =1+(2-\alpha) x+(2-\beta) y+\cdots,
\end{aligned}
$$

\footnotetext{
${ }^{43}$ In sheaf theoretic terms, $\operatorname{Pic}(C)=H^{1}\left(C, \mathcal{O}_{C}^{*}\right)$.

${ }^{44}$ In App. H, we correspondingly associate two sheaves to a divisor $D=\sum a_{i} p_{i} \cdot \mathcal{O}(D)$ is the sheaf of meromorphic sections of $[D]$ with poles of degree $\leq a_{i}$ at $p_{i}$ for each $a_{i}<0$; and $\mathcal{O}(-D)$ is the sheaf of meromorphic sections of $[D]$ with zeros of degree $\geq a_{i}$ at $p_{i}$ for each $a_{i}>0$.
} 
so,

$$
\begin{aligned}
\chi\left(B_{\alpha, \beta}\right) & =\int_{B_{\alpha, \beta}} c_{1}\left(T_{B_{\alpha, \beta}}\right)=\int_{\mathbb{P}^{1} \times \mathbb{P}^{1}} c_{1}\left(T_{B_{\alpha, \beta}}\right) \wedge c_{1}\left(N_{B_{\alpha, \beta}}\right) \\
& =2-2(\alpha-1)(\beta-1) .
\end{aligned}
$$

On the other hand, $\chi=2-2 g$ for a genus $g$ curve. Therefore,

$$
g=(\alpha-1)(\beta-1)
$$

\section{H Direct image functor}

Given a continuous map of topological spaces $f: X \rightarrow Y$, the direct image functor, used in the proof of the Calabi-Yau condition in Eq. (4.11) and App. I, provides a map from sheaves on $X$ to sheaves on $Y$. The purpose of this appendix is to provide background on the direct image functor and higher direct images. For completeness, we begin with the definition of a sheaf. For a more complete discussion, the reader is referred to Secs. 0.3, 1.1, and 3.5 of Ref. [28], which we follow closely.

A presheaf $\mathcal{F}$ on a topological space $X$ assigns a set $\mathcal{F}(U)$ to each open set $U \subset X$, as well as a restriction map $r_{U, V}: \mathcal{F}(U) \rightarrow \mathcal{F}(V)$ to each pair $U \subset V$. The restriction map is required to satisfy $r_{W, U}=r_{V, U} \circ r_{W, V}$ for $U \subset V \subset W$. We will assume below that the $\mathcal{F}(U)$ are abelian groups.

Then, $\mathcal{F}$ is a sheaf, provided that the following conditions are satisfied.

1. Sections $\sigma_{1} \in \mathcal{F}(U)$ and $\sigma_{2} \in \mathcal{F}(V)$ whose restrictions agree over the intersection $U \cap V$ are the restrictions of a unique section over the union $U \cup V$ :

$$
\left.\sigma_{1}\right|_{U \cap V}=\left.\sigma_{2}\right|_{U \cap V} \Rightarrow \exists \rho \in \mathcal{F}(U \cup V) \text { such that }\left.\rho\right|_{U}=\sigma_{1} \text { and }\left.\rho\right|_{V}=\sigma_{2}
$$

2. If $\sigma \in \mathcal{F}(U \cup V)$ and $\left.\sigma\right|_{U}=\left.\sigma\right|_{V}=0$, then $\sigma=0$.

Common sheaves that we might consider are:

The locally constant sheaves $\mathbb{Z}, \mathbb{Q}, \mathbb{R}, \mathbb{C}$, with $\mathbb{Z}(U)=$ additive group of locally constant $\mathbb{Z}$-valued functions on $U$, and similar definitions for $\mathbb{Q}, \mathbb{R}$, and $\mathbb{C}$.

The structure sheaf $\mathcal{O}$, with $\mathcal{O}(U)=$ additive group of holomorphic functions on $U$.

The sheaf $\mathcal{O}^{*}$, with $\mathcal{O}^{*}(U)=$ multiplicative group of nowhere vanishing holomorphic functions on $U$. 
The sheaf $\mathcal{O}(D)$. Given a divisor $D=\sum a_{i} V_{i}$ in terms of irreducible hypersurfaces $V_{i}$, and its corresponding line bundle $[D]$, we define $\mathcal{O}(D)=$ additive group of meromorphic sections of $[D]$, with poles of order $\leq a_{i}$ on $V_{i}$.

The sheaf $\Omega^{p}$, with $\Omega^{p}(U)=$ additive group of holomorphic $p$-forms on $U$.

Maps of sheaves of abelian groups are group homomorphisms compatible with the sheaf conditions on the restriction maps. Čech cohomology gives a definition of the cohomology of sheaves. We refer the reader to Ref. [28] for a description of Čech cohomology. For our purposes, it should suffice to note that (i) such a cohomology can be defined, and (ii) it agrees with de Rham and singular cohomology for the locally constant sheaves $\mathbb{R}$ and $\mathbb{Z}$, respectively. Likewise, the Čech cohomology of $\Omega^{p}$ agrees with Dolbeault cohomology:

$$
H^{q}\left(X, \Omega^{p}\right) \cong H_{\bar{\partial}}^{p, q}
$$

Given a continuous map $f: X \rightarrow Y$ of topological spaces and a sheaf $\mathcal{F}: U \rightarrow \mathcal{F}(U)$, a natural map of sheaves is the direct image functor $f_{*}$, defined by

$$
f_{*} \mathcal{F}: V \rightarrow \mathcal{F}\left(f^{-1}(V)\right),
$$

for $V$ an open set in $Y$. Likewise, we define the higher direct images $R^{q} f_{*}$ by

$$
R^{q} f_{*} \mathcal{F}: V \rightarrow H^{q}\left(f^{-1}(V), \mathcal{F}\right) .
$$

The ' $R$ ' stands for right derived functor. ${ }^{45}$

For a fibration $F \rightarrow X \stackrel{f}{\rightarrow} \mathcal{B}$ with generic fiber $F$, and $U \subset \mathcal{B}$ an open set of the base, the open set $f^{-1}(U) \cong U \times F$ contains the entire fiber over each point of $U$. Thus, to first approximation $R^{q} f_{*}(\mathbb{Q})$ is the constant sheaf $H^{q}(F, \mathbb{Q})$ - the $q$ th cohomology group along the fiber (for contractible $U$ ). We then need to modify this approximation to take into account the monodromy action of the fundamental group of $\mathcal{B}$ on the cycles in $H^{q}(F, \mathbb{Q})$.

This definition via a first approximation followed by corrections can be made precise in terms of a Leray spectral sequence $\left\{E_{r}^{p, q}\right\}$ with $E_{\infty} \Rightarrow H^{*}(X, \mathcal{F})$ whose second step is

$$
E_{2}^{p, q}=H^{p}\left(\mathcal{B}, R^{q} f_{*} \mathcal{F}\right)
$$

as explained on p. 462-468 of Ref. [28]. For example, in de Rham cohomology, we have

$$
E_{2}^{p, q}=H_{\mathrm{DR}}^{p}\left(\mathcal{B}, H_{\mathrm{DR}}^{q}(F)\right),
$$

\footnotetext{
${ }^{45}$ The direct image functor is left exact, but not necessarily right exact. However, under mild assumptions, there is a canonical way to extend the sequence to the right to form a long exact sequence, by appending "right derived functors" of $\mathcal{F}$. These right derived functors are precisely the higher direct images.
} 
where the right hand side is defined by viewing $H_{\mathrm{DR}}^{q}(F)$ as a vector bundle over $\mathcal{B}$ associated to a representation of the fundamental group.

In general, the cohomology rings $H^{*}(X, \mathcal{F})$ and $H^{*}\left(\mathcal{B}, R^{*} f_{*} \mathcal{F}\right)$ need not agree, however, under further restrictions this is the case. For $\mathcal{F}=\mathbb{Q}$, and $X$ and $B$ compact and Kähler, the sequence $\left\{E_{r}^{p, q}\right\}$ can be shown to converge at the second step, so that

$$
E_{2} \cong E_{\infty} \quad \text { and } \quad H^{*}(X, \mathbb{Q}) \cong H^{*}\left(\mathcal{B}, R^{*} f_{*} \mathbb{Q}\right)
$$

In this case, the $H^{p}\left(\mathcal{B}, R^{q} f_{*} \mathbb{Q}\right)$ give a filtration of $H^{*}(X, \mathbb{Q})$ according to base and fiber degree, much like the Dolbeault cohomology gives a filtration of the de Rham cohomolog according to holomorphic and antiholomorphic degree.

\section{Proof of the Calabi-Yau condition}

To evaluate Eq. (4.11), it is convenient to factorize the projection map $\rho: S \rightarrow \mathbb{P}_{u, v}^{1}$ as $\rho=f \circ \varphi$, where $\varphi$ is the double cover $\varphi: S \rightarrow \mathbb{P}_{s, t}^{1} \times \mathbb{P}_{u, v}^{1}$, and we define $f$ and $g$ to be the projection maps $f: \mathbb{P}_{s, t}^{1} \times \mathbb{P}_{u, v}^{1} \rightarrow \mathbb{P}_{u, v}^{1}$ and $g: \mathbb{P}_{s, t}^{1} \times \mathbb{P}_{u, v}^{1} \rightarrow \mathbb{P}_{s, t}^{1}$. Then,

$$
\rho_{*} K_{S / \mathbb{P}^{1}}=\left(R^{1} \rho_{*} \mathcal{O}_{S}\right)^{*}=\left(R^{1} f_{*} \varphi_{*} \mathcal{O}_{S}\right)^{*},
$$

where we have used Serre duality on the genus-2 fibers in the first equality. For a double cover $\varphi: A \rightarrow B$, we have the general result ${ }^{46}$

$$
\varphi_{*} \mathcal{O}_{A}=\mathcal{O}_{B} \oplus \mathcal{O}_{B}\left(-\frac{1}{2}(\text { branch locus })\right) \text {. }
$$

In our case, this gives $\varphi_{*} \mathcal{O}_{S}=\mathcal{O}_{\mathbb{P}^{1} \times \mathbb{P}^{1}} \oplus \mathcal{O}_{\mathbb{P}^{1} \times \mathbb{P}^{1}}(-3,-1)$, from which

$$
R^{i} \rho_{*} \mathcal{O}_{S}=R^{i} f_{*} \mathcal{O}_{\mathbb{P}^{1} \times \mathbb{P}^{1}} \oplus R^{i} f_{*} \mathcal{O}_{\mathbb{P}^{1} \times \mathbb{P}^{1}}(-3,-1) .
$$

The first term is

$$
R^{i} f_{*} \mathcal{O}_{\mathbb{P}^{1} \times \mathbb{P}^{1}}=\left\{\begin{array}{cl}
\mathcal{O}_{\mathbb{P}^{1}} & i=0 \\
0 & i=1
\end{array}\right.
$$

and the second is

$$
\begin{aligned}
R^{i} f_{*} \mathcal{O}_{\mathbb{P}^{1} \times \mathbb{P}^{1}}(-3,-1) & =R^{i} f_{*}\left(f^{*} \mathcal{O}_{\mathbb{P}^{1}}(-1) \otimes g^{*} \mathcal{O}_{\mathbb{P}^{1}}(-3)\right) \\
& =\mathcal{O}_{\mathbb{P}^{1}}(-1) \otimes R^{i} f_{*} g^{*} \mathcal{O}_{\mathbb{P}^{1}}(-3) \\
& =\mathcal{O}_{\mathbb{P}^{1}}(-1) \otimes g_{0}^{*} R^{i} f_{0 *} \mathcal{O}_{\mathbb{P}^{1}}(-3) \\
& =\mathcal{O}_{\mathbb{P}^{1}}(-1) \otimes H^{i}\left(\mathbb{P}^{1}, \mathcal{O}(-3)\right) \\
& =\left\{\begin{array}{cc}
0 & i=0, \\
\mathcal{O}_{\mathbb{P}^{1}}(-1) \oplus \mathcal{O}_{\mathbb{P}^{1}}(-1) & i=1 .
\end{array}\right.
\end{aligned}
$$

\footnotetext{
${ }^{46}$ For example, for the double cover $\varphi: \mathbb{P}^{1} \rightarrow \mathbb{P}^{1}, z \mapsto z^{2}$, we have branch points at 0 and $\infty$, and $\varphi_{*} \mathcal{O}_{\mathbb{P}^{1}}=\mathcal{O}_{\mathbb{P}^{1}} \oplus \mathcal{O}_{\mathbb{P}^{1}}(-1)$. For $\varphi: E \rightarrow \mathbb{P}^{1}$, with $E$ an elliptic curve, we have four branch points, and $\varphi_{*} \mathcal{O}_{E}=\mathcal{O}_{\mathbb{P}^{1}} \oplus \mathcal{O}_{\mathbb{P}^{1}}(-2)$.
} 
Here, $f_{0}$ and $g_{0}$ are maps from $\mathbb{P}^{1}$ to a point $p$, so that we have the following commutative diagram:

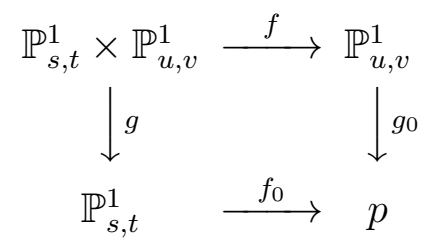

In the last line of Eq. (I.4), we have used $H^{0}\left(\mathbb{P}^{1}, \mathcal{O}(-3)\right)=0$, and by Serre duality ${ }^{47}$ $H^{1}\left(\mathbb{P}^{1}, \mathcal{O}(-3)\right)=H^{0}\left(\left(\mathbb{P}^{1}, \mathcal{O}(1)\right)\right)^{*}=\mathbb{C} \oplus \mathbb{C}$. Combining results (4.11) through (I.4), we obtain $N_{\mathbb{P}^{1}}=\mathcal{O}_{\mathbb{P}^{1}}(-1) \oplus \mathcal{O}_{\mathbb{P}^{1}}(-1)$, as claimed. The Calabi-Yau condition follows.

\section{J Intersections of theta surfaces of $\mathcal{X}$}

In this appendix, we define the theta surfaces $\Theta_{I}, \Theta_{I}^{\prime}$ of Sec. 4.3.1, and compute their double and triple intersections in $\mathcal{X}$, as well as their intersections with the abelian surface fiber $A$.

An outline of the construction of the theta surfaces is as follows. The genus- 2 fibration $S \rightarrow \mathbb{P}^{1}$ has $2 \times 12=24$ sections $\ell_{I}, \ell_{I}^{\prime}$. Each defines an isomorphism

$$
\operatorname{Pic}^{1}\left(S / \mathbb{P}^{1}\right) \stackrel{\cong}{\rightarrow} \operatorname{Pic}^{0}\left(S / \mathbb{P}^{1}\right)=\mathcal{X}
$$

Once one such isomorphism has been chosen, each section $\ell_{I}$ or $\ell_{I}^{\prime}$ of $S$ defines a corresponding theta surface $\Theta_{I}$ or $\Theta_{I}^{\prime}$, respectively, embedding $S$ in the Calabi-Yau manifold $\mathcal{X}$.

The correspondence is most easily understood fiberwise. Therefore, we first focus on a single genus-2 curve $C$. In this case, each point $p$ on the curve defines a theta divisor $C_{p}$ of the Jacobian abelian surface $A$, which is an embedding of $C$ in $A$. We compute the intersections of these $C_{p}$. Then, we fiber this construction over $\mathbb{P}^{1}$. This replaces $C$ by $S$, $p$ by a section $\ell$ of $S$, and $A$ by the relative Jacobian $\mathcal{X}$ of $S$. The double intersections in $A$ become curves in $\mathcal{X}$, and we use this intermediate result to compute the desired triple intersections in $\mathcal{X}$.

\section{Warm-up: intersections of curves in an abelian surface}

Let us first review the map between points of a complex curve and theta divisors of its Jacobian, and then use this map to compute intersections the theta divisors in the genus- 2 case, where the Jacobian is an abelian surface. For background on complex curves $C$, their Jacobians $\mathcal{J}_{C}$, and spaces of degree $d$ line bundles $\operatorname{Pic}^{d}(C)$, the reader is referred to App. F.

Let $C$ be any curve of genus $g$. $\operatorname{Pic}^{g-1}(C)$ has a canonical $\Theta$-divisor,

$$
\Theta=\left\{L \in \operatorname{Pic}^{g-1}(C) \mid h^{0}(L)>0\right\} .
$$

\footnotetext{
${ }^{47}$ For $E$ a vector bundle and $\operatorname{dim} M=n$, Serre duality states that $H^{p}(M, E)=H^{n-p}\left(M, K \otimes E^{*}\right)^{*}$.
} 
More generally, for all $d \geq 0$, consider the variety

$$
W_{d}=\left\{L \in \operatorname{Pic}^{d}(C) \mid h^{0}(L)>0\right\}
$$

Its dimension is $\min (d, g)$. When $d=g-1, W_{d}$ is a divisor of $\operatorname{Pic}^{d}(C)$. Otherwise it is a subvariety of other dimension. Now, given a line bundle $L \in \operatorname{Pic}^{1}(C)$, we have an isomorphism $\operatorname{Pic}^{d}(C) \cong \operatorname{Pic}^{0}(C)$, realized by tensoring with $L^{-\otimes d}$, so that $W_{d}$ can also be viewed as a subvariety of $\operatorname{Pic}^{0}(C)$. Therefore, a line bundle $L \in \operatorname{Pic}^{g-1}(C)$ determines a theta divisor $\Theta_{L} \subset \operatorname{Pic}^{0}(C)$. In the case of interest $g=2$, the result is as follows:

A line bundle $L \in \operatorname{Pic}^{1}(C)$ determines a theta divisor $\Theta_{L} \subset \operatorname{Pic}^{0}(C)$, embedding the genus-2 curve $C$ in its Jacobian surface $\mathcal{J}_{C}=\operatorname{Pic}^{0}(C)$.

In the genus- 2 case, intersections of theta divisors in the abelian surface $A=\operatorname{Pic}^{0}(C)$ are computed as follows. Let us focus on line bundles $L=\mathcal{O}(p)$, where $p$ is a point on the genus- 2 curve $C$, and denote the corresponding theta divisors (embeddings $C \hookrightarrow A$ ) by $C_{p}$. Given two points $p_{1}, p_{2} \in C$, we have

$$
\begin{aligned}
C_{p_{1}} \cap_{A} C_{p_{2}} & =\left\{L \in \operatorname{Pic}^{0}(C) \mid h^{0}\left(L \otimes \mathcal{O}\left(p_{1}\right)\right)>0, h^{0}\left(L \otimes \mathcal{O}\left(p_{2}\right)\right)>0\right\} \\
& =\mathcal{O}\left(-p_{1}\right) \otimes\left\{q \in C \mid h^{0}\left(\mathcal{O}\left(q+p_{2}-p_{1}\right)\right)>0\right\} \quad\left(\mathcal{O}(q) \approx L \otimes \mathcal{O}\left(p_{1}\right)\right) \\
& =\mathcal{O}\left(-p_{1}\right) \otimes\left\{\mathcal{O}\left(p_{1}\right), \mathcal{O}\left(p_{2}^{\prime}\right)\right\}, \\
& =\left\{\mathcal{O}(0), \mathcal{O}\left(p_{2}^{\prime}-p_{1}\right)\right\} \quad \text { for } \quad p_{1} \neq p_{2} .
\end{aligned}
$$

Here, $p^{\prime}=\imath(p)=h-p$, where $\imath$ denotes the hyperelliptic involution on the genus- 2 curve (sending one branch to the other) and $h$ is independent of $p$. For $p_{1}=p_{2}$, the intersection is the whole curve $C_{p_{1}}=C_{p_{2}}$. We write this result simply as

$$
C_{p_{1}} \cap_{A} C_{p_{2}}= \begin{cases}\left\{0, p_{2}^{\prime}-p_{1}\right\} & p_{1} \neq p_{2} \\ C_{p_{1}} & p_{1}=p_{2}\end{cases}
$$

Note that $p_{2}^{\prime}-p_{1}=p_{1}^{\prime}-p_{2}$, so that the intersection is symmetric. For $p_{1}=p_{2}^{\prime}$, the intersection consists of the single point $0 \in A$, counted with multiplicity 2 for the homological intersection.

\section{Intersections of pairs of surfaces in the threefold $\mathcal{X}$}

Next, consider the genus-2 fibration $S$ and its relative Jacobian $\mathcal{X}=\operatorname{Pic}^{0}\left(S / \mathbb{P}^{1}\right)$. In this case, the analog of the theta curve $C_{p}$ associated to each point $p \in C$ is a family of genus-2 curves $C_{\ell}$ associated to each section $\ell$ of the genus- 2 fibration. In the same way that each $p$ determined a line bundle $\mathcal{O}(p) \in \operatorname{Pic}^{1}(C)$ above, each section $\ell$ now determines a section of $\operatorname{Pic}^{1}\left(S / \mathbb{P}^{1}\right)$.

Let $\Theta_{I}, \Theta_{I}^{\prime}$ denote the total space associated to the family $C_{\ell_{I}}, C_{\ell_{I}^{\prime}}$, respectively. Each of these theta surfaces is an embedding of $S$ in the Calabi-Yau threefold $\mathcal{X}$. From the previous 
result (J.5), the intersections of pairs of distinct theta surfaces are

$$
\begin{array}{ll}
\Theta_{I} \cdot \Theta_{J}=\sigma_{0}+\sigma_{\ell_{I}^{\prime}-\ell_{J}}, & \Theta_{I}^{\prime} \cdot \Theta_{J}^{\prime}=\sigma_{0}+\sigma_{\ell_{I}-\ell_{J}^{\prime}} \\
\Theta_{I}^{\prime} \cdot \Theta_{J}=\sigma_{0}+\sigma_{\ell_{I}-\ell_{J}}, & \Theta_{I} \cdot \Theta_{I}^{\prime}=2 \sigma_{0}+C_{\ell_{I} \cap \ell_{I}^{\prime}} .
\end{array}
$$

Here, $L \rightarrow \sigma_{L}$ is the isomorphism identifying degree zero line bundles in $\operatorname{Pic}^{0}\left(S / \mathbb{P}^{1}\right)$ with sections of the abelian fibered threefold $\mathcal{X}$. Note that $\ell_{I}^{\prime}-\ell_{J}=\ell_{J}^{\prime}-\ell_{I}$ as a consequence of Eq. (4.5). The curve $C_{\ell_{I} \cap \ell_{I}^{\prime}}$ is the common genus-2 fiber of $\Theta_{I}$ and $\Theta_{I}^{\prime}$. For self intersections, we have

$$
\Theta_{I} \cdot \Theta_{I}=c_{1}\left(K_{\Theta_{I}}\right) .
$$

Recall that $C$ is the generic genus-2 fiber of the projection $S \rightarrow \mathbb{P}_{u, v}^{1}$ and $C^{\prime}$ is the generic genus-0 fiber of the projection $S \rightarrow \mathbb{P}_{s, t}^{1}$. Since $\Theta_{I} \cong S$, the relevant fact for applying the last result to the computation of triple intersections is

$$
c_{1}\left(K_{S}\right)=C^{\prime}-C .
$$

This result follows from the double cover formula $K_{S}=\pi^{*}\left(K_{\mathbb{P}^{1} \times \mathbb{P}^{1}} \otimes L\right)$ (cf. Lemma. 17.1 in Ref. [5]). Here, $L^{2}$ is defined by the branch curve $B$ of the double cover $S \rightarrow \mathbb{P}^{1} \times \mathbb{P}^{1}$ as in Sec. 4.1:

$$
L^{2}=\mathcal{O}_{\mathbb{P}^{1} \times \mathbb{P}^{1}}(B)=\mathcal{O}(6,2) .
$$

This gives

$$
\begin{aligned}
K_{S} & =\pi^{*}\left(K_{\mathbb{P}^{1} \times \mathbb{P}^{1}} \otimes \mathcal{O}(3,1)\right), \\
c_{1}\left(K_{S}\right) & =-2\left(C+C^{\prime}\right)+\left(3 C^{\prime}+C\right)=C^{\prime}-C .
\end{aligned}
$$

\section{Triple intersections of divisors of $\mathcal{X}$}

Finally, the triple intersections of divisors in $\mathcal{X}$ can obtained as double intersections of curves in surfaces. For example, for $I, J, K$ distinct,

$$
\begin{aligned}
\Theta_{I} \cdot \Theta_{J} \cdot \Theta_{K} & =\left(\Theta_{I} \cdot \Theta_{J}\right) \cdot \Theta_{J}\left(\Theta_{J} \cdot \Theta_{K}\right) \\
& =\left(\sigma_{0}+\sigma_{\ell_{I}^{\prime}-\ell_{J}}\right) \cdot \Theta_{J}\left(\sigma_{0}+\sigma_{\ell_{K}^{\prime}-\ell_{J}}\right) \\
& \cong\left(\ell_{J}+\ell_{I}^{\prime}\right) \cdot{ }_{S}\left(\ell_{J}+\ell_{K}^{\prime}\right) \\
& =-1 .
\end{aligned}
$$

Here, we have used the fact that $\sigma_{\ell-\ell_{J}}$ maps to $\ell \in S$ under the isomorphism $\Theta_{J} \rightarrow S$. The remaining triple intersections of theta surfaces are

$$
\begin{aligned}
& \Theta_{I} \cdot \Theta_{J} \cdot \Theta_{J}=\Theta_{I} \cdot \Theta_{J}^{\prime} \cdot \Theta_{J}^{\prime}=-2, \\
& \Theta_{I} \cdot \Theta_{I} \cdot \Theta_{I}^{\prime}=\Theta_{I} \cdot \Theta_{J} \cdot \Theta_{J}^{\prime}=0, \\
& \Theta_{I} \cdot \Theta_{I} \cdot \Theta_{I}=-4,
\end{aligned}
$$


together with equations obtained from these by exchange of $\Theta$ and $\Theta^{\prime}$. The computation is analogous to the previous one. Using Eq. (J.6), it is possible to confirm that the result is independent of choice of which of the three theta surfaces is used to perform the double intersection.

We now turn to intersections involving the generic abelian surface fiber $A$. In this case, $A^{2}=0$, and

$$
A \cdot \Theta_{I} \cdot \Theta_{J}=A \cdot \Theta_{I} \cdot \Theta_{J}^{\prime}=A \cdot \Theta_{I}^{\prime} \cdot \Theta_{J}^{\prime}=2,
$$

for any $I, J$, not necessarily distinct. This is most easily proven from the intersection of curves in the abelian fiber $A$. For example,

$$
\begin{aligned}
A \cdot \Theta_{I} \cdot \Theta_{J} & =\left(A \cdot \Theta_{I}\right) \cdot{ }_{A}\left(A \cdot \Theta_{J}\right) \\
& \cong C \cdot{ }_{A} C=2
\end{aligned}
$$

as desired. (In an abelian surface, the self-intersection of a genus- $g$ curve is $2 g-2$.)

The same result is obtained if the intersections are performed in a theta surface. Let $C_{I}$ denote the genus-2 fiber of $\Theta_{I} \cong S$. Then, for example, for $I \neq J$,

$$
\begin{aligned}
A \cdot \Theta_{I} \cdot \Theta_{J} & =\left(A \cdot \Theta_{I}\right) \cdot \Theta_{I}\left(\Theta_{I} \cdot \Theta_{J}\right) \\
& =C_{I} \cdot \Theta_{I}\left(\sigma_{0}+\sigma_{\ell_{J}^{\prime}-\ell_{I}}\right) \\
& \cong C \cdot S\left(\ell_{I}+\ell_{J}^{\prime}\right)=2, \\
A \cdot \Theta_{I} \cdot \Theta_{I} & =\left(A \cdot \Theta_{I}\right) \cdot \Theta_{I}\left(\Theta_{I} \cap \Theta_{I}\right) \\
& =C \cdot \Theta_{I} c_{1}\left(K_{\Theta_{I}}\right) \\
& \cong C \cdot S\left(C^{\prime}-C\right)=2 .
\end{aligned}
$$

In the last step, we have used the fact that the genus-2 fiber $C$ of $S$ satisfies $C^{2}=0$ and $C \cdot C^{\prime}=C \cdot\left(\ell_{K}+\ell_{K}^{\prime}\right)=2$.

\section{References}

[1] P. C. Argyres and M. R. Douglas, "New phenomena in $S U(3)$ supersymmetric gauge theory," Nucl. Phys. B 448, 93 (1995) [arXiv:hep-th/9505062].

[2] P. S. Aspinwall, "An analysis of fluxes by duality," arXiv:hep-th/0504036.

[3] T. Banks, "Matrix theory," Nucl. Phys. Proc. Suppl. 67, 180 (1998) [arXiv:hepth/9710231].

[4] T. Banks, M. R. Douglas and N. Seiberg, "Probing F-theory with branes," Phys. Lett. B 387, 278 (1996) [arXiv:hep-th/9605199]. 
[5] W. P. Barth, K. Hulek, C. Peters and A. Van de Ven, "Compact Complex Surfaces," (Springer Verlag, New York 2000).

[6] E. Bergshoeff, R. Kallosh, A. K. Kashani-Poor, D. Sorokin and A. Tomasiello, "An index for the Dirac operator on D3 branes with background fluxes," JHEP 0510, 102 (2005) [arXiv:hep-th/0507069].

[7] N. Bolis and M. B. Schulz, "Toward warped Kaluza-Klein reduction," work in progress.

[8] V. Bouchard and R. Donagi, "An $S U(5)$ heterotic standard model," Phys. Lett. B 633, 783 (2006) [arXiv:hep-th/0512149].

[9] V. Bouchard and R. Donagi, "On a class of non-simply connected Calabi-Yau threefolds," arXiv:0704.3096 [math.AG].

[10] C. P. Burgess, P. G. Camara, S. P. de Alwis, S. B. Giddings, A. Maharana, F. Quevedo and K. Suruliz, "Warped supersymmetry breaking," JHEP 0804, 053 (2008) [arXiv:hepth/0610255].

[11] P. G. Camara and E. Dudas, "Multi-instanton and string loop corrections in toroidal orbifold models," JHEP 0808, 069 (2008) [arXiv:0806.3102 [hep-th]].

[12] P. Candelas and R. Davies, "New Calabi-Yau Manifolds with Small Hodge Numbers," arXiv:0809.4681 [hep-th].

[13] D. A. Cox and S. Zucker, "Intersection numbers of Sections of Elliptic Surfaces," Inventiones Math. 53, 1-44 (1979).

[14] A. Dabholkar and C. Hull, "Duality twists, orbifolds, and fluxes," JHEP 0309, 054 (2003) [arXiv:hep-th/0210209].

[15] O. DeWolfe and B. Zwiebach, "String junctions for arbitrary Lie algebra representations," Nucl. Phys. B 541, 509 (1999) [arXiv:hep-th/9804210].

[16] O. DeWolfe, "Affine Lie algebras, string junctions and 7-branes," Nucl. Phys. B 550, 622 (1999) [arXiv:hep-th/9809026].

[17] O. DeWolfe, T. Hauer, A. Iqbal and B. Zwiebach, "Uncovering the symmetries on $(p, q)$ 7-branes: Beyond the Kodaira classification," Adv. Theor. Math. Phys. 3, 1785 (1999) [arXiv:hep-th/9812028].

[18] O. DeWolfe, T. Hauer, A. Iqbal and B. Zwiebach, "Uncovering infinite symmetries on $(p, q)$ 7-branes: Kac-Moody algebras and beyond," Adv. Theor. Math. Phys. 3, 1835 (1999) [arXiv:hep-th/9812209]. 
[19] O. DeWolfe and S. B. Giddings, "Scales and hierarchies in warped compactifications and brane worlds," Phys. Rev. D 67, 066008 (2003) [arXiv:hep-th/0208123].

[20] R. Donagi and M.-H. Saito, work in progress.

[21] A. R. Frey and J. Polchinski, "N $\mathcal{N}=3$ warped compactifications," Phys. Rev. D 65, 126009 (2002) [arXiv:hep-th/0201029].

[22] A. R. Frey and A. Maharana, "Warped spectroscopy: Localization of frozen bulk modes," JHEP 0608, 021 (2006) [arXiv:hep-th/0603233].

[23] M. Fukae, Y. Yamada and S. K. Yang, "Mordell-Weil lattice via string junctions," Nucl. Phys. B 572, 71 (2000) [arXiv:hep-th/9909122].

[24] O. J. Ganor, "On zeroes of superpotentials in F-theory," Nucl. Phys. Proc. Suppl. 67, 25 (1998).

[25] P. Gao and M. B. Schulz, work in progress.

[26] S. B. Giddings and A. Maharana, "Dynamics of warped compactifications and the shape of the warped landscape," Phys. Rev. D 73, 126003 (2006) [arXiv:hep-th/0507158].

[27] D. Green, A. Lawrence, J. McGreevy, D. R. Morrison and E. Silverstein, "D(imensional) Duality," Phys. Rev. D 76, 066004 (2007) [arXiv:0705.0550 [hep-th]].

[28] P. Griffiths and J. Harris, "Principles of Algebraic Geometry," (Wiley, New York 1978).

[29] M. Gross and S. Popescu, "Calabi-Yau Threefolds and Moduli of Abelian Surfaces I" I. Compositio Math. 127, p. 169-228 (2000) [arXiv:math/0001089].

[30] L. Borisov and Z. Hua, "On Calabi-Yau threefolds with large nonabelian fundamental groups," Proc. Amer. Math. Soc. 136 (2008), 1549-1551 [arXiv:math/0609728].

[31] M. Gross, "Some Calabi-Yau manifolds with small Betti numbers," talk presented at String Phenomenology 2008, University of Pennsylvania, 31 May 2008 [http://www.math.upenn.edu/StringPhenom2008/talks.html].

[32] A. Hanany and E. Witten, "Type IIB superstrings, BPS monopoles, and threedimensional gauge dynamics," Nucl. Phys. B 492, 152 (1997) [arXiv:hep-th/9611230].

[33] S. Hellerman, J. McGreevy and B. Williams, "Geometric constructions of nongeometric string theories," JHEP 0401, 024 (2004) [arXiv:hep-th/0208174].

[34] M. Hindry and J. S. Silverman, Diaphantine Geometry: An Introduction, Graduate Texts in Mathematics, No. 201, Springer-Verlag, New York (2001) 
[35] T. Hübsch, "Calabi-Yau manifolds: A Bestiary for physicists," Singapore: World Scientific (1992) $362 \mathrm{p}$.

[36] C. M. Hull, "A geometry for non-geometric string backgrounds," JHEP 0510, 065 (2005) [arXiv:hep-th/0406102].

[37] S. Kachru, M. B. Schulz and S. Trivedi, "Moduli stabilization from fluxes in a simple IIB orientifold," JHEP 0310, 007 (2003) [arXiv:hep-th/0201028].

[38] S. Kachru, M. B. Schulz, P. K. Tripathy and S. P. Trivedi, "New supersymmetric string compactifications," JHEP 0303, 061 (2003) [arXiv:hep-th/0211182].

[39] A. Lawrence, M. B. Schulz and B. Wecht, "D-branes in nongeometric backgrounds," JHEP 0607, 038 (2006) [arXiv:hep-th/0602025].

[40] K. Oguiso and T. Shioda, "The Mordell-Weil Lattice of a Rational Elliptic Surface," Comment. Math. Univ. St. Paul. 40 (1991) 83.

[41] M.-H. Saito and K.-I. Sakakibara, "On Mordell-Weil lattices of higher genus fibrations on rational surfaces," J. Math. Kyoto Univ., 34 (1994), 859-871.

[42] M.-H. Saito, "Prepotentials of Yukawa couplings of certain Calabi-Yau 3-folds and mirror symmetry," The arithmetic and geometry of algebraic cycles (Banff, AB, 1998), 385-425, NATO Sci. Ser. C Math. Phys. Sci., 548, Kluwer Acad. Publ., Dordrecht (2000).

[43] M.-H. Saito, "A Calabi-Yau threefold with abelian fibration and $\mathbb{Z} / 2 \mathbb{Z}$ action," talk presented at String Phenomenology 2008, University of Pennsylvania, 29 May 2008 [http://www.math.upenn.edu/StringPhenom2008/talks.html].

[44] A. Saltman and E. Silverstein, "The scaling of the no-scale potential and de Sitter model building," JHEP 0411, 066 (2004) [arXiv:hep-th/0402135].

[45] M. B. Schulz, "Superstring orientifolds with torsion: O5 orientifolds of torus fibrations and their massless spectra," Fortsch. Phys. 52, 963 (2004) [arXiv:hep-th/0406001].

[46] M. B. Schulz, "Calabi-Yau duals of torus orientifolds," JHEP 0605, 023 (2006) [arXiv:hep-th/0412270].

[47] N. Seiberg and E. Witten, "Electric-magnetic duality, monopole condensation, and confinement in $\mathcal{N}=2$ supersymmetric Yang-Mills theory," Nucl. Phys. B 426, 19 (1994) [Erratum-ibid. B 430, 485 (1994)] [arXiv:hep-th/9407087]. 
[48] A. Sen, "A note on enhanced gauge symmetries in M- and string theory," JHEP 9709, 001 (1997) [arXiv:hep-th/9707123].

[49] J. Shelton, W. Taylor and B. Wecht, "Nongeometric flux compactifications," JHEP 0510, 085 (2005) [arXiv:hep-th/0508133].

[50] T. Shioda, "Theory of Mordell-Weil lattices," in Proceedings of the International Congress of Mathematicians (Kyoto 1990), Math. Soc. Japan (1991) 473-489.

[51] T. Shioda, "On the Mordell-Weil lattices," Comment. Math. Univ. St. Paul. 39 (1990) 211-240.

[52] B. Spanjaard, "Compactifications of IIA supergravity on $S U(2)$-structure manifolds,"

[53] G. Shiu, G. Torroba, B. Underwood and M. R. Douglas, "Dynamics of Warped Flux Compactifications," JHEP 0806, 024 (2008) [arXiv:0803.3068 [hep-th]].

[54] A. Tomasiello, "Topological mirror symmetry with fluxes," JHEP 0506, 067 (2005) [arXiv:hep-th/0502148].

[55] C. Vafa, "Evidence for F-Theory," Nucl. Phys. B 469, 403 (1996) [arXiv:hepth/9602022].

[56] D. Vegh and J. McGreevy, "Semi-Flatland," arXiv:0808.1569 [hep-th].

[57] Wikipedia contributors, "Elliptic surface," Wikipedia: The Free Encyclopedia, http://en.wikipedia.org/wiki/Elliptic_surface [Online; accessed 2 June 2008] 